\title{
Disk, interval, point: on constructions of quantum field theories with holomorphic action functionals
}

\author{
Nafiz Ishtiaque ${ }^{a, b}$ and Junya Yagi ${ }^{b}$ \\ ${ }^{a}$ Institute for Advanced Study, \\ Princeton, NJ, U.S.A. \\ ${ }^{b}$ Perimeter Institute for Theoretical Physics, \\ Waterloo, ON, Canada \\ E-mail: nishtiaque@ias.edu, jyagi@perimeterinstitute.ca
}

ABSTRACT: Bosonic quantum field theories with holomorphic action functionals are realized by two types of constructions involving supersymmetric quantum field theories, compactified on an interval in one type and compactified on a disk and deformed in the other. We establish the equivalence between the two types of constructions by reducing the disk to the interval and the interval to a point. As examples, we discuss constructions of zerodimensional gauged sigma model, gauged quantum mechanics, gauged symplectic bosons in two dimensions, and Chern-Simons theory and its higher-dimensional variants.

KEYwords: Extended Supersymmetry, Supersymmetric Gauge Theory, Topological Field Theories

ARXIV EPRINT: 2002.10488 


\section{Contents}

1 Introduction 1

2 B-model, supersymmetric quantum mechanics and zero-dimensional sigma model

$2.1 \Omega$-deformation of the B-model $\quad 6$

$\begin{array}{ll}2.2 & \text { Reduction to supersymmetric quantum mechanics } \\ & 10\end{array}$

$\begin{array}{lll}2.3 & \text { Cigar reduction of the } \Omega \text {-deformed B-model } & 14\end{array}$

$\begin{array}{lll}2.4 & \text { Reduction to zero-dimensional sigma model } & 16\end{array}$

$\begin{array}{ll}2.5 \text { Multivalued superpotentials } & 17\end{array}$

$\begin{array}{ll}2.6 & \text { Lagrangian branes from the gradient flow } \\ & 18\end{array}$

3 Rozansky-Witten theory, A-model and quantum mechanics 19

$\begin{array}{ll}3.1 & \text { Rozansky-Witten theory } \\ 3.2 & 19\end{array}$

3.2 B-model formulation of Rozansky-Witten theory 21

$3.3 \Omega$-deformed Rozansky-Witten theory and quantum mechanics 23

3.4 Reduction to the A-model 25

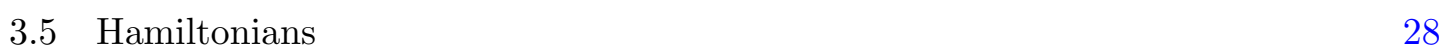

4 Gauge symmetry $\quad 30$

$4.1 \Omega$-deformed gauged B-model $\quad 30$

4.2 Reduction to supersymmetric gauged quantum mechanics 34

$\begin{array}{lll}4.3 & \text { Boundary conditions } & 36\end{array}$

4.4 Reduction to zero-dimensional gauged sigma model $\quad 37$

5 Gauge theory applications $\quad 39$

$\begin{array}{ll}5.1 \text { Gauged quantum mechanics } & 39\end{array}$

5.2 Gauged symplectic bosons 40

5.3 Chern-Simons theory $\quad 45$

5.4 Higher-dimensional Chern-Simons theories 48

A Eight-dimensional super Yang-Mills theory as $\mathcal{N}=(2,2)$ supersymmetric gauge theory 51

\section{Introduction}

The purpose of this paper is to build a bridge between two types of constructions of quantum field theories, typically bosonic ones, from supersymmetric quantum field theories in higher spacetime dimensions. These constructions have appeared in such diverse contexts as quantization of symplectic manifolds $[1,2]$, vertex algebras in three- $[3,4]$ and 
four-dimensional [5-7] supersymmetric field theories, analytic continuation of knot invariants $[8,9]$ and their categorification [10], the $3 \mathrm{~d}-3 \mathrm{~d}$ correspondence [11-18], and emergence of integrable systems from supersymmetric gauge theories [19-30].

We will refer the two types of constructions as the A-type and the B-type. There are some similarities between them, but also crucial differences.

In an A-type construction, one starts with a $(d+1)$-dimensional supersymmetric field theory, formulated on a product $\mathbb{I} \times \mathbb{M}_{d}$, where $\mathbb{I}$ is an interval $[0, \ell]$ and $\mathbb{M}_{d}$ is a $d$-manifold. The theory is topologically twisted, either fully or partially, so that it is topological on $\mathbb{I}$. Let $s$ be the coordinate on $\mathbb{I}$.

At one end of $\mathbb{I}$, say at $s=0$, one imposes Neumann-like boundary conditions on the bosonic matter fields. At the other end, at $s=\ell$, boundary conditions constrain the bosonic matter fields to be, roughly speaking, valued in a Lagrangian submanifold $M$ of their Kähler ${ }^{1}$ target space $Y$.

Since the theory is topological on $\mathbb{I}$, one can shorten $\mathbb{I}$ until it collapses to a point. One thus obtains a $d$-dimensional theory on $\mathbb{M}_{d}$. It turns out that the action functional of this theory on $\mathbb{M}_{d}$ is holomorphic in complex-valued bosonic fields, but the path integral makes sense for appropriate choices of $M$ as it is performed over a middle-dimensional cycle determined by $M$.

In a prototypical example [1] of an A-type construction, $d=1$ and the two-dimensional theory on $\mathbb{I} \times \mathbb{M}_{1}$ is the A-model [31], which may be obtained from two-dimensional $\mathcal{N}=$ $(2,2)$ supersymmetric sigma model by the A-twist. The target space $Y$ is a complex symplectic manifold. Located at $s=0$ is a canonical coisotropic brane [32], supported on all of $Y$. At $s=\ell$, one chooses a brane whose support $M$ is Lagrangian with respect to two of the real symplectic structures of $Y$, but symplectic with respect to another. The one-dimensional theory on $\mathbb{M}_{1}$ is quantum mechanics whose phase space is $M$, regarded as a symplectic manifold.

More elaborate examples arise for $d \geq 2$.

For $d=2$, one may take the three-dimensional theory to be an $\mathcal{N}=4$ supersymmetric field theory in the A-twist [33] (which is the "mirror" of the Rozansky-Witten twist). The two-dimensional theory produced by reduction on $\mathbb{I}$ is a chiral conformal field theory (CFT) on a Riemann surface $\mathbb{M}_{2}[3,4]$. If the theory one picks is a gauge theory constructed from vector multiplets and hypermultiplets in a complex symplectic representation $Y$ of the gauge group, the CFT is the system of gauged symplectic bosons valued in $Y$.

An example with $d=3$ is provided by the GL-twist [34-36] of $\mathcal{N}=4$ super Yang-Mills theory in four dimensions. In this case, the three-dimensional theory is Chern-Simons theory on a three-manifold $\mathbb{M}_{3}$, whose gauge group is the complexification $H_{\mathbb{C}}$ of the gauge group $H$ of the Yang-Mills theory [1, 8, 9].

A B-type construction starts with a $(d+2)$-dimensional supersymmetric field theory on $\mathbb{D} \times \mathbb{M}_{d}$, where $\mathbb{D}$ is a disk. The construction involves a special kind of deformation of the theory, called the $\Omega$-deformation [37, 38]. The theory is topologically twisted so

\footnotetext{
${ }^{1}$ More generally, $Y$ may be complex and almost symplectic but non-Kähler. We will consider such target spaces in sections 2 and 3.
} 
that it is topological on $\mathbb{D}$, but the $\Omega$-deformation reduces the topological invariance to the invariance under deformations that leave the rotation symmetry of $\mathbb{D}$ unbroken. On the boundary $\partial \mathbb{D}$ of the disk, one imposes a boundary condition such that the bosonic matter fields take values in a Lagrangian submanifold $M$ of the Kähler target space $Y$.

As in A-type constructions, one can shrink $\mathbb{D}$ to a point to obtain a $d$-dimensional theory on $\mathbb{M}_{d}$. Again, the resulting theory turns out to have a holomorphic action functional, and the path integral is performed over a middle-dimensional cycle specified by $M$.

In an example [2] with $d=1$, the three-dimensional theory on $\mathbb{D} \times \mathbb{M}_{1}$ is RozanskyWitten theory [39], which arises from $\mathcal{N}=4$ supersymmetric sigma model by the B-twist (or the Rozansky-Witten twist). The target space $Y$ is a complex symplectic manifold, and the boundary condition on $\partial \mathbb{D}$ requires the bosonic field to lie in a submanifold $M$ that is either Lagrangian or symplectic, depending on which real symplectic structure one refers to. The one-dimensional theory on $\mathbb{M}_{1}$ that arises after the $\Omega$-deformation and reduction on $\mathbb{D}$ is quantum mechanics, quantizing the symplectic manifold $M$.

For an example with $d=2$, one may consider Kapustin's holomorphic-topological twist [40] of an $\mathcal{N}=2$ supersymmetric field theory in four dimensions. The $\Omega$-deformation and reduction on $\mathbb{D}$ result in a chiral CFT, which, for a gauge theory with hypermultiplets in a complex symplectic representation $Y$, is the system of gauged symplectic bosons valued in $Y[6,7]$.

Finally, for $d=3$, the $\Omega$-deformation and reduction of a topological twist of fivedimensional $\mathcal{N}=2$ super Yang-Mills theory with gauge group $H$ yields Chern-Simons theory with gauge group $H_{\mathbb{C}}[18]$.

Above we have described examples of A-type and B-type constructions for $d=1,2,3$. The parallel between the two types is conspicuous: for every $d$, there is a pair of A-type and B-type constructions for one and the same $d$-dimensional bosonic theory.

This observation suggests that for each $d$, the theory used in the A-type construction should be somehow related to the one used in the corresponding B-type construction. The expectation is further strengthened if one notices the fact that the two theories may be obtained by topological twist from two physical theories with the same amount of supersymmetry.

In fact, there is a natural way to produce a $(d+1)$-dimensional theory on $\mathbb{I} \times \mathbb{M}_{d}$ from a $(d+2)$-dimensional theory on $\mathbb{D} \times \mathbb{M}_{d}$ : one deforms the disk $\mathbb{D}$ into the shape of a cigar and performs circle reduction of the latter theory, considering the cigar as a circle fibration over the interval $\mathbb{I}$. Such a deformation of $\mathbb{D}$ is allowed in the theory for the B-type construction since it preserves rotation symmetry. A similar reduction has been studied before in a different but related setting [41].

Presumably, the B-type theory turns into its A-type counterpart through the $\Omega$ deformation and the cigar reduction of $\mathbb{D}$ to $\mathbb{I}$. In this paper we demonstrate that this is indeed true.

Our strategy is to first establish the correspondence between A-type and B-type constructions in the most basic case, namely the case in which $d=0$. Then, we apply the results to higher-dimensional examples. 
For $d=0$, the two-dimensional theory relevant for B-type construction is the gauged B-model [42] on $\mathbb{D}$, which may originate from $\mathcal{N}=(2,2)$ supersymmetric gauged sigma model via the B-twist $[43,44]$. The target space of the model is a Kähler manifold $X$ with a holomorphic $G_{\mathbb{C}^{-} \text {action, where }} G$ is the gauge group. To this theory we apply the $\Omega$-deformation $[2,18]$. On $\partial \mathbb{D}$, we place a brane whose support $L$ is $G_{\mathbb{C} \text {-invariant and }}$ defines a Lagrangian submanifold $\mathcal{L}$ inside the Kähler quotient $X / / G$.

We will show that upon cigar reduction, the $\Omega$-deformed gauged B-model on $\mathbb{D}$ becomes topologically twisted supersymmetric gauged quantum mechanics whose target space is $X$. The theory thus obtained on $\mathbb{I}$ is the A-type theory for $d=0$. The brane on $\partial \mathbb{D}$ descends to a similar boundary condition at $s=\ell$, while the center of $\mathbb{D}$, or the "tip" of the cigar, becomes the boundary at $s=0$ where the Neumann-like boundary conditions for the bosonic matter fields emerge.

We will also show that in the limit where $\mathbb{I}$ shrinks to a point, this supersymmetric gauged quantum mechanics reduces to a gauged sigma model on a point $\mathbb{M}_{0}$ with target $L$, provided that $L$ is chosen appropriately. This is the bosonic theory for $d=0$. Remarkably, the gauge group complexifies to $G_{\mathbb{C}}$ in the process of this reduction.

To establish the link between the A-type and B-type constructions for $d \geq 1$, we merely apply the results we have obtained for $d=0$ to various infinite-dimensional target spaces. The point is that the B-type theory on $\mathbb{D} \times \mathbb{M}_{d}$ may be regarded as the gauged B-model on $\mathbb{D}$, whereas the A-type theory on $\mathbb{I} \times \mathbb{M}_{d}$ may be regarded as supersymmetric gauged quantum mechanics on $\mathbb{I}$, and the two have the same target space $X$. The bosonic theory on $\mathbb{M}_{d}$ may be viewed as a zero-dimensional gauged sigma model with target $L$.

For example, for $d=1$, we take $X=\operatorname{Map}\left(\mathbb{M}_{1}, Y\right)$, the space of maps from $\mathbb{M}_{1}$ to a complex symplectic manifold $Y$. For the brane on $\partial \mathbb{D}$, we choose an appropriate submanifold $M \subset Y$ and set $L=\operatorname{Map}\left(\mathbb{M}_{1}, M\right)$. The A-model on $\mathbb{I} \times \mathbb{M}_{1}$ with target $Y$ is topologically twisted supersymmetric quantum mechanics on $\mathbb{I}$ with target $X$, while Rozansky-Witten theory on $\mathbb{D} \times \mathbb{M}_{1}$ with target $Y$ is the B-model on $\mathbb{D}$ with target $X$. Hence, these two theories are related by cigar reduction, and realize the same zero-dimensional sigma model with target $L .^{2}$ The last theory describes maps from $\mathbb{M}_{1}$ to $M$, so it is quantum mechanics with phase space $M$.

Similarly, for $d=2$, we take $X=\operatorname{Map}\left(\mathbb{M}_{2}, Y\right)$, the space of maps from $\mathbb{M}_{2}$ to the vector space $Y$ for a complex symplectic representation of a gauge group. For $d=3$, we take $X$ to be the space of $H_{\mathbb{C}}$ gauge fields on $\mathbb{M}_{3}$. The relation between the A-type and B-type constructions for $d=1,2,3$ are thus established.

If one knows either an A-type or B-type construction of a certain bosonic theory, one may exploit the relation just explained to arrive at the corresponding construction of the other type. In this way we will deduce an A-type construction of a four-dimensional variant of Chern-Simons theory [26-28] based on five-dimensional $\mathcal{N}=2$ super Yang-Mills theory, starting from the B-type construction using six-dimensional $\mathcal{N}=(1,1)$ super Yang-Mills theory [30]. This A-type construction was essentially proposed in [45, 46].

\footnotetext{
${ }^{2}$ We have learned from Dylan Butson that this statement and related results may also be understood from the point of view of equivariant factorization algebras.
} 


\begin{tabular}{|lccc|}
\hline$d$ & Bosonic theory & A-type theory & B-type theory \\
\hline 0 & Sigma model & Topological SQM & B-model \\
1 & Quantum mechanics & A-model & Rozansky-Witten \\
2 & CFT & A-twisted $\mathcal{N}=4$ & Kapustin \\
3 & Chern-Simons & GL-twisted $\mathcal{N}=4$ SYM & Topological $\mathcal{N}=2$ SYM \\
4 & 4 d Chern-Simons & Twisted $\mathcal{N}=2$ SYM & Twisted $\mathcal{N}=(1,1)$ SYM \\
5 & 5 d Chern-Simons & Twisted $\mathcal{N}=(1,1)$ SYM & Twisted SYM \\
6 & 6d Chern-Simons & Twisted SYM & Twisted SYM \\
\hline
\end{tabular}

Table 1. Examples of A-type and B-type constructions. SQM and SYM are abbreviations for "supersymmetric quantum mechanics" and "super Yang-Mills theory," respectively.

In the examples with $d=3,4$ described above, $d$-dimensional Chern-Simons theory is realized by $(d+1)$ - and $(d+2)$-dimensional maximally supersymmetric Yang-Mills theories. This pattern continues to hold for $d=5,6$, and we will explain the A-type and B-type constructions in these cases. Six-dimensional Chern-Simons theory is more commonly known as holomorphic Chern-Simons theory [47]. Five-dimensional Chern-Simons theory was introduced in [48].

Table 1 summarizes the A-type and B-type constructions treated in this paper.

In concluding this introduction, a few of remarks are in order.

First, for the constructions of higher-dimensional Chern-Simons theories to be completely satisfactory, super Yang-Mills theories in dimension greater than four should probably be provided with ultraviolet completion. One way to do so is to embed them into string theory using branes. This approach proves to be fruitful, as it allows one to exploit the rich structure of dualities in string theory [10,30].

Second, it seems that a large class of bosonic gauge theories with holomorphic action functionals and complex gauge groups admit A-type and B-type constructions, at least formally, since they can always be reformulated as zero-dimensional gauged sigma models. What distinguishes the examples we consider is that the corresponding A-type and B-type theories are physically natural and interesting. In contrast, the A-type and B-type theories will not be so nice if one takes a generic bosonic gauge theory. Their actions will lack Lorentz invariance and contain higher-derivative terms.

Last, although our treatment of infinite-dimensional target spaces may seem naive, it is justified. These spaces are essentially the field spaces of the bosonic theories on $\mathbb{M}_{d}$. In general, the definition of a quantum field theory comes with a regularization of ultraviolet divergences. Whatever the choice of a regularization we make for a bosonic theory, we use the same regularization for the corresponding A-type and B-type theories. For instance, one may latticize $\mathbb{M}_{d}$; the twisted supercharges are compatible with a lattice regularization since they do not generate translations on $\mathbb{M}_{d}$. Then, the field space becomes the product of copies of a finite-dimensional space.

This paper is organized as follows. We begin in section 2 by studying the A-type and B-type constructions for $d=0$ without gauge symmetry. Then, in section 3 , we apply these constructions to demonstrate the equivalences between the $\Omega$-deformed Rozansky- 
Witten theory, the A-model and quantum mechanics. We incorporate gauge symmetry into the picture in section 4. Finally, in section 5, we discuss constructions of gauged quantum mechanics, gauged symplectic bosons, and Chern-Simons theory and its higher-dimensional variants by supersymmetric gauge theories. Appendix A explains the formulation of eightdimensional super Yang-Mills theory as an $\mathcal{N}=(2,2)$ supersymmetric gauge theory in two dimensions.

\section{B-model, supersymmetric quantum mechanics and zero-dimensional sigma model}

In this section we discuss the fundamental A-type and B-type constructions, which realize sigma model on a point $\mathbb{M}_{0}$ within supersymmetric quantum mechanics on an interval $\mathbb{I}$ and the $\Omega$-deformed B-model on a cigar $\mathbb{D}$, respectively. To alleviate technicalities, we will not consider gauge symmetry yet.

After we formulate the $\Omega$-deformed B-model with a complex target space, we will show that its circle reduction gives supersymmetric quantum mechanics. Then, we will discuss boundary conditions in the respective theories, and reduction to a zero-dimensional theory. Finally, we will explain how to construct good boundary conditions using a gradient flow.

\section{$2.1 \Omega$-deformation of the B-model}

The B-model is a topological quantum field theory of cohomological type, and may be constructed from $\mathcal{N}=(2,2)$ supersymmetric sigma model by the B-type topological twist [43, 44]. For the presence of $\mathcal{N}=(2,2)$ supersymmetry, the target space of the model must be a Kähler manifold, and for the B-twist to make sense, it must moreover be Calabi-Yau. If, however, one does not require the B-model to originate from a physical theory via topological twist, the target space can be more general.

For us, the target space is a complex manifold $X$ with complex structure $I$, which is not necessarily Kähler. We still require its first Chern class to vanish, $c_{1}(X)=0$, so that the theory suffers no anomalies. If $X$ is a Kähler manifold, this requirement means that $X$ is Calabi-Yau. We will use letters $\mu, \nu, \ldots$ for real indices, $i, j, \ldots$ for holomorphic indices, and $\bar{\imath}, \bar{\jmath}, \ldots$ for antiholomorphic indices.

The spacetime (or worldsheet) of the B-model is a surface $\Sigma$, endowed with a Riemannian metric $\gamma$. In this paper we will take $\Sigma$ to have rotation symmetry and $\gamma$ to be rotation invariant.

The final input data of the B-model is the superpotential $W$, which is a holomorphic function on $X$.

The fields of the B-model are a bosonic field

$$
\varphi \in \operatorname{Map}(\Sigma, X)
$$


and fermionic fields

$$
\begin{aligned}
& \eta \in \Pi \Omega^{0}\left(\Sigma, \varphi^{*} T^{0,1} X\right), \\
& \rho \in \Pi \Omega^{1}\left(\Sigma, \varphi^{*} T^{1,0} X\right), \\
& \mu \in \Pi \Omega^{2}\left(\Sigma, \varphi^{*} T^{0,1} X\right) .
\end{aligned}
$$

Here $\Omega^{p}(\Sigma, E)$ is the space of $p$-forms on $\Sigma$ with values in the vector bundle $E$ over $\Sigma$, and $T^{1,0} X$ and $T^{0,1} X$ are the holomorphic and antiholomorphic tangent bundles of $X$, respectively; $\Pi$ denotes parity reversal. In the off-shell formulation which we will employ, the theory also has auxiliary bosonic two-form fields

$$
\begin{aligned}
& \mathrm{G} \in \Omega^{2}\left(\Sigma, \varphi^{*} T^{1,0} X\right), \\
& \overline{\mathrm{G}} \in \Omega^{2}\left(\Sigma, \varphi^{*} T^{0,1} X\right) .
\end{aligned}
$$

All of these fields come from a chiral multiplet of $\mathcal{N}=(2,2)$ supersymmetry.

The B-model has supersymmetry generated by a fermionic conserved charge $Q_{0}$. The supercharge $Q_{0}$ squares to zero,

$$
Q_{0}^{2}=0,
$$

and is used to define cohomology in the space of states and in the space of operators. The path integral of the theory, with $Q_{0}$-closed operators inserted on $\Sigma$ and $Q_{0}$-closed states specified on $\partial \Sigma$, depends only on the $Q_{0}$-cohomology classes of those operators and states.

The essential point is that the metric $\gamma$ on $\Sigma$ enters the theory only through $Q_{0}$-exact terms in the action. As a consequence, under deformations of $\gamma$, the integrand of the path integral varies by $Q_{0}$-exact terms and its $Q_{0}$-cohomology class remains intact. Thus, by passing to $Q_{0}$-cohomology, the theory becomes invariant under deformations of $\gamma$. In this sense the B-model is a topological theory.

We will not explain here how $Q_{0}$ acts on the fields and how the action functional of the B-model is constructed. Rather, we directly proceed to describe the $\Omega$-deformation of the B-model.

The $\Omega$-deformation of the B-model [2] is a deformation that may be applied whenever $\Sigma$ admits an isometry. Let $V$ be a Killing vector field generating this isometry. The $\Omega-$ deformed B-model has deformed supersymmetry generated by a supercharge $Q_{V}$. The $\Omega$-deformed supercharge $Q_{V}$ reduces to $Q_{0}$ for $V=0$, and squares to the generator $\mathcal{L}_{V}$ of the isometry:

$$
Q_{V}^{2}=\mathcal{L}_{V}
$$

On fields, $\mathcal{L}_{V}$ acts by the Lie derivative by $V$.

Slightly more generally, we allow $V$ to be a complex linear combination of Killing vector fields, provided that it commutes with its complex conjugate $\bar{V}$ :

$$
[V, \bar{V}]=0 .
$$


This condition ensures that $\mathcal{L}_{V}$ commutes with $\iota_{\bar{V}} \cdot{ }^{3}$ The fact that $V$ generates a complexified isometry implies that $\mathcal{L}_{V}$ commutes with the Hodge star operator $\star$.

As in the case of the ordinary B-model, in the $\Omega$-deformed B-model one considers the $Q_{V}$-cohomologies of states and operators. The difference is that in the $\Omega$-deformed case, one has to restrict the action of the supercharge to $V$-invariant states and operators, for only in the spaces of such states and operators does one have the relation $Q_{V}^{2}=0$. By restriction to the $Q_{V}$-invariant sector, the $\Omega$-deformed B-model becomes quasi-topological: the path integral is invariant under deformations of $\gamma$ as long as $V$ remains as a Killing vector field.

Now we describe the $\Omega$-deformation more explicitly.

The field content of the $\Omega$-deformed B-model is the same as that of the ordinary Bmodel. On the fields, $Q_{V}$ acts by the variations

$$
\begin{aligned}
\delta \varphi^{i} & =\iota_{V} \rho^{i}, \\
\delta \rho^{i} & =\mathrm{d} \varphi^{i}+\iota_{V} \mathrm{G}^{i}, \\
\delta \mathrm{G}^{i} & =\mathrm{d} \rho^{i}, \\
\delta \bar{\varphi}^{\bar{\imath}} & =\eta^{\bar{\imath}}, \\
\delta \eta^{\bar{\imath}} & =V\left(\bar{\varphi}^{\bar{\imath}}\right), \\
\delta \mu^{\bar{\imath}} & =\overline{\mathrm{G}}^{\bar{\imath}}, \\
\delta \overline{\mathrm{G}}^{\bar{\imath}} & =\mathrm{d} \iota_{V} \mu^{\bar{\imath}},
\end{aligned}
$$

where we have described the map $\varphi$ locally on $X$ with a tuple of complex functions $\left(\varphi^{i}, \bar{\varphi}^{\bar{\imath}}\right)$, corresponding to holomorphic and antiholomorphic coordinates. We see that the vector field $\delta_{Q_{V}}$ in the field space representing the action of $Q_{V}$ satisfies

$$
\delta_{Q_{V}}^{2}=\mathrm{d} \iota_{V}+\iota_{V} \mathrm{~d} .
$$

The right-hand side is the Lie derivative by $V$ on differential forms.

While the transformations (2.10)-(2.16) satisfy the desired supersymmetry algebra, they are not covariant under diffeomorphisms of $X$ because $\delta_{Q_{V}}$ and $\mathrm{d}$ are not covariant derivatives. For the construction of the model, it will be more convenient to rewrite the above formulas in manifestly covariant forms. This can be achieved as follows.

Choose a torsion-free connection $\nabla$ on $X$ that preserves the complex structure:

$$
\nabla I=0 .
$$

The connection coefficients $\Gamma$ of $\nabla$ are symmetric,

$$
\Gamma_{\nu \rho}^{\mu}=\Gamma_{\rho \nu}^{\mu},
$$

\footnotetext{
${ }^{3}$ A quick way to see this is to note that since $V+\bar{V}$ and $\mathrm{i}(V-\bar{V})$ are two commuting real vector fields, one can find local coordinates $(x, y)$ such that $V=\partial_{x}+\mathrm{i} \partial_{y}$ (or $V=c \partial_{x}$ for some $c \in \mathbb{C}$ if $V$ and $\bar{V}$ are linearly dependent). In terms of these coordinates, $\mathcal{L}_{V}$ acts on tensors by $\partial_{x}+\mathrm{i} \partial_{y}$, and the commutativity is obvious.
} 
and has no mixed components, that is,

$$
\Gamma_{\nu \rho}^{\mu}=0
$$

unless $\mu, \nu, \rho$ are all holomorphic or all antiholomorphic indices. When $X$ is Kähler, many of the formulas that follow will simplify greatly if one chooses $\nabla$ to be the Levi-Civita connection associated with the Kähler metric.

Using $\nabla$, we define the covariant exterior derivative $\mathrm{d}_{\nabla}$ as the exterior derivative $\mathrm{d}$ coupled to the pullback of $\nabla$ by $\varphi$. For example,

$$
\mathrm{d}_{\nabla} \rho^{i}=\mathrm{d} \rho^{i}+\mathrm{d} \varphi^{k} \Gamma_{k j}^{i} \wedge \rho^{j} .
$$

Likewise, we define the covariant variation by

$$
\delta_{\nabla} \rho^{i}=\delta \rho^{i}+\delta \varphi^{k} \Gamma_{k j}^{i} \wedge \rho^{j}
$$

and so on. Also, we introduce new auxiliary fields $\mathrm{F}^{i}, \overline{\mathrm{F}}^{\bar{\imath}}$ by

$$
\begin{aligned}
& \mathrm{F}^{i}=\mathrm{G}^{i}+\frac{1}{2} \Gamma_{j k}^{i} \rho^{j} \wedge \rho^{k}, \\
& \overline{\mathrm{F}}^{\bar{\imath}}=\overline{\mathrm{G}}^{\bar{\imath}}+\Gamma_{\bar{\jmath} \bar{k}}^{\bar{\imath}} \eta^{\bar{j}} \mu^{\bar{k}} .
\end{aligned}
$$

Then, the variations (2.10)-(2.16) can be written covariantly as

$$
\begin{aligned}
\delta \varphi^{i} & =\iota_{V} \rho^{i}, \\
\delta_{\nabla} \rho^{i} & =\mathrm{d} \varphi^{i}+\iota_{V} \mathrm{~F}^{i}, \\
\delta_{\nabla} \mathrm{F}^{i} & =\mathrm{d}_{\nabla} \rho^{i}-\left(\frac{1}{3}\left(R_{\nabla}\right)^{i}{ }_{j k l} \iota_{V} \rho^{l}+\frac{1}{2}\left(R_{\nabla}\right)^{i}{ }_{j k \bar{l}} \eta^{\bar{l}}\right) \rho^{j} \wedge \rho^{k}, \\
\delta \bar{\varphi}^{\bar{\imath}} & =\eta^{\bar{\imath}}, \\
\delta_{\nabla} \eta^{\bar{\imath}} & =V\left(\bar{\varphi}^{\bar{\imath}}\right), \\
\delta_{\nabla} \mu^{\bar{\imath}} & =\overline{\mathrm{F}}^{\bar{\imath}}, \\
\delta_{\nabla} \overline{\mathrm{F}}^{\bar{\imath}} & =\mathrm{d}_{\nabla} \iota_{V} \mu^{\bar{\imath}}-\left(\left(R_{\nabla}\right)^{\bar{j}}{ }_{\bar{\jmath} \bar{l} l} \iota_{V} \rho^{l}+\frac{1}{2}\left(R_{\nabla}\right)^{\bar{i}} \overline{\bar{k} \bar{l}} \eta^{\bar{l}}\right) \eta^{\bar{\jmath}} \mu^{\bar{k}} .
\end{aligned}
$$

Here $R_{\nabla}$ is the curvature tensor for $\nabla$ :

$$
\left(R_{\nabla}\right)^{\mu}{ }_{\nu \rho \sigma}=\partial_{\rho} \Gamma_{\sigma \nu}^{\mu}-\partial_{\sigma} \Gamma_{\rho \nu}^{\mu}+\Gamma_{\rho \tau}^{\mu} \Gamma_{\rho \nu}^{\tau}-\Gamma_{\sigma \tau}^{\mu} \Gamma_{\rho \nu}^{\tau} .
$$

In particular, $\left(R_{\nabla}\right)^{i}{ }_{j k l}=\partial_{k} \Gamma_{l j}^{i}-\partial_{l} \Gamma_{k j}^{i}+\Gamma_{k m}^{i} \Gamma_{l j}^{m}-\Gamma_{l m}^{i} \Gamma_{k j}^{m}$ and $\left(R_{\nabla}\right)^{i}{ }_{j k \bar{l}}=-\partial_{\bar{l}} \Gamma_{k j}^{i}$.

To write down the action $S_{\Omega \mathrm{B}}$ for the $\Omega$-deformed B-model, we pick a Riemannian metric $g$ on $X$ that is compatible with the complex structure. The action is a sum of two pieces:

$$
S_{\Omega \mathrm{B}}=S_{\Omega \mathrm{B}, \mathrm{C}}+S_{\Omega \mathrm{B}, W}
$$

The main part $S_{\Omega \mathrm{B}, \mathrm{C}}$ of the action is $Q_{V}$-exact and contains the kinetic terms:

$$
S_{\Omega \mathrm{B}, \mathrm{C}}=\delta_{Q_{V}} \int_{\Sigma} g_{i \bar{\jmath}}\left(\rho^{i} \wedge \star\left(\mathrm{d} \bar{\varphi}^{\bar{\jmath}}+\iota \bar{V}^{\overline{\mathrm{F}}}\right)+\mathrm{F}^{i} \wedge \star \mu^{\bar{\jmath}}\right) .
$$

This is $Q_{V}$-invariant because $\mathcal{L}_{V}$ commutes with $\star$ and $\iota_{\bar{V}}$, and the integrand is $V$-invariant. 
The second piece $S_{\Omega \mathrm{B}, W}$ is constructed from the superpotential. Assume that $V$ is nonvanishing on the boundary of $\Sigma$ (which is a collection of circles if $\Sigma$ is compact), and let $\theta$ be a coordinate on $\partial \Sigma$. Then,

$$
S_{\Omega \mathrm{B}, W}=\int_{\Sigma}\left(\mathrm{F}^{i} \partial_{i} W+\frac{1}{2} \rho^{i} \wedge \rho^{j} \nabla_{i} \partial_{j} W-\delta_{Q_{V}}\left(\mu^{\bar{\imath}} \partial_{\bar{\imath}} \bar{W}\right)\right)-\int_{\partial \Sigma} W \frac{\mathrm{d} \theta}{V^{\theta}}
$$

The $Q_{V}$-invariance of $S_{\Omega \mathrm{B}, W}$ is easily checked if $S_{\Omega \mathrm{B}, W}$ is expressed with the original auxiliary fields $\mathrm{G}, \overline{\mathrm{G}}$.

Although the above action depends on the choice of the connection $\nabla$, the $Q_{V}$-invariant sector of the theory does not. Written in terms of $G$ and $\bar{G}$, the supersymmetry transformations (2.10)-(2.16) are independent of $\nabla$, while the action depends on $\nabla$ only through $Q_{V}$-exact terms. By the same token, the $Q_{V}$-invariant sector is independent of the choice of the target metric $g$.

\subsection{Reduction to supersymmetric quantum mechanics}

As a preliminary step to understanding the cigar reduction of the $\Omega$-deformed B-model, let us establish the relation between the circle reduction of the $\Omega$-deformed B-model and supersymmetric quantum mechanics. In the following analysis we will not take into account the effects of the boundary of $\Sigma$.

Suppose that the B-model is placed on the product $\Sigma=\mathbb{R} \times \mathbb{S}^{1}$ of the real line $\mathbb{R}$ and a circle $\mathbb{S}^{1}$, endowed with coordinates $(s, \theta)$ and a rotation invariant metric

$$
\gamma(s, \theta)=\gamma_{s s}(s) \mathrm{d} s^{2}+\gamma_{\theta \theta}(s) \mathrm{d} \theta^{2}
$$

and we apply the $\Omega$-deformation with respect to the vector field

$$
V=\epsilon \partial_{\theta}
$$

generating rotations, where $\epsilon$ is a complex constant. We will use the indices $\hat{s}, \hat{\theta}$ to denote components of tensors with respect to the orthonormal vectors $\partial_{\hat{s}}=\sqrt{\gamma^{s s}} \partial_{s}, \partial_{\hat{\theta}}=\sqrt{\gamma^{\theta \theta}} \partial_{\theta}$ and one-forms $\mathrm{d} \hat{s}=\sqrt{\gamma_{s s}} \mathrm{~d} s, \mathrm{~d} \hat{\theta}=\sqrt{\gamma_{\theta \theta}} \mathrm{d} \theta$. In this notation, the norm $\|V\|$ of $V$ is equal to $\left|V^{\hat{\theta}}\right|$.

In general, the path integral whose integrand is supersymmetric localizes to the field configurations such that the supersymmetry variations of fermions vanish: away from this locus in the field space, the parameter of supersymmetry transformations serves as a fermionic coordinate, but the supersymmetric integrand is by definition independent of this coordinate and hence the Grassmannian integration vanishes. Since $\delta_{Q_{V}}\left(\iota_{V} \rho^{i}\right)=V\left(\varphi^{i}\right)$ and $\delta_{Q_{V}} \eta^{\bar{\imath}}=V\left(\bar{\varphi}^{\bar{\imath}}\right)$, in the case at hand the path integral localizes to rotation invariant maps. This means that the $\Omega$-deformed B-model on $\mathbb{R} \times \mathbb{S}^{1}$ can be described as a onedimensional theory on $\mathbb{R}$. 
To understand this reduction to one dimension in a more down-to-earth manner, we can add to the action the $Q_{V}$-exact terms

$$
\begin{aligned}
\delta_{Q_{V}} \int_{\mathbb{R} \times \mathbb{S}^{1}} u g_{i \bar{\jmath}}\left(\bar{V}\left(\varphi^{i}\right) \wedge \star \eta^{\bar{\jmath}}+\mathcal{L}_{V} \mathrm{~F}^{i} \wedge \star \mathcal{L}_{\bar{V}} \mu^{\bar{\jmath}}\right) \\
=\int_{\mathbb{R} \times \mathbb{S}^{1}} u g_{i \bar{\jmath}}\left(\bar{V}\left(\varphi^{i}\right) \wedge \star V\left(\bar{\varphi}^{\bar{\jmath}}\right)+\mathcal{L}_{V} \mathrm{~F}^{i} \wedge \star \mathcal{L}_{\bar{V}} \overline{\mathrm{F}}^{\bar{\jmath}}\right. \\
\left.\quad+\bar{V}\left(\iota_{V} \rho^{i}\right) \wedge \star \eta^{\bar{\jmath}}+\mathcal{L}_{V} \mathrm{~d} \rho^{i} \wedge \star \mathcal{L}_{\bar{V}} \mu^{\bar{\jmath}}\right)+\cdots,
\end{aligned}
$$

where $\cdots$ indicates higher-order terms. Expand each field $\Psi$ in Fourier modes along $\mathbb{S}^{1}$ as $\Psi=\sum_{n \in \mathbb{Z}} \Psi_{n} e^{i n \theta}$. If we take the limit $u \rightarrow \infty$, the above modification gives infinitely large mass to all nonzero modes $\Psi_{n}, n \neq 0$, thereby suppressing their contributions. Thus, we are left with only the zero modes $\Psi_{0}$, which describe fields in the one-dimensional theory.

We now show that this one-dimensional theory is supersymmetric quantum mechanics with target space $X$, in the presence of a potential that is determined by $W$.

The version of supersymmetric quantum mechanics that we will find is a topologically twisted one, relevant for Morse theory [49]. The theory consists of bosonic fields

$$
\begin{aligned}
& \phi \in \operatorname{Map}(\mathbb{R}, X), \\
& \mathrm{H} \in \Omega^{1}\left(\mathbb{R}, \phi^{*} T X\right)
\end{aligned}
$$

and fermionic fields

$$
\begin{aligned}
& \psi \in \Pi \Omega^{0}\left(\mathbb{R}, \phi^{*} T X\right), \\
& \chi \in \Pi \Omega^{1}\left(\mathbb{R}, \phi^{*} T X\right),
\end{aligned}
$$

and has supersymmetry transforming them as

$$
\begin{aligned}
\delta \phi^{\mu} & =\psi^{\mu}, \\
\delta_{\nabla^{\prime}} \psi^{\mu} & =0 \\
\delta_{\nabla^{\prime}} \chi^{\mu} & =\mathrm{d} \phi^{\mu}+\mathrm{iH}^{\mu}, \\
\delta_{\nabla^{\prime}} \mathrm{H}^{\mu} & =\mathrm{id}_{\nabla^{\prime}} \psi^{\mu}-\frac{\mathrm{i}}{2}\left(R_{\nabla^{\prime}}\right)^{\mu}{ }_{\nu \rho \sigma} \chi^{\nu} \psi^{\rho} \psi^{\sigma} .
\end{aligned}
$$

Here $\nabla^{\prime}$ is any torsion-free connection on $X$. If one wishes, one could absorb $\nabla^{\prime}$ by a redefinition of the auxiliary field $\mathrm{H}$.

The action of the theory depends on a real function $h$ and a flat abelian gauge field $a$ on $X$. It is given by

$$
S_{\mathrm{SQM}}=\frac{1}{\hbar} \delta_{Q} \int_{\mathbb{R}} \frac{1}{2} g_{\mu \nu} \chi^{\mu} \star\left(\mathrm{d} \phi^{\nu}-\mathrm{i} \mathrm{H}^{\nu}\right)+\frac{1}{\hbar} \int_{\mathbb{R}}\left(-\delta_{Q}\left(\chi^{\mu} \partial_{\mu} h\right)+\mathrm{d} h+\mathrm{i} \phi^{*} a\right),
$$

where $\hbar$ is the Planck constant and $\delta_{Q}$ denotes the supersymmetry variation. The flatness of $a$ is necessary for the action to be supersymmetric. (As mentioned already, we neglect the effects of the boundary of $\mathbb{R}$. Appropriate boundary conditions are assumed so that $\int_{\mathbb{R}} \mathrm{d} h$ is supersymmetric.) 
The equations of motion for $\mathrm{H}$ is

$$
\mathrm{H}_{\hat{s}}^{\mu}=\mathrm{i} g^{\mu \nu} \partial_{\nu} h+\frac{\mathrm{i}}{2} \delta \phi^{\rho} g^{\mu \sigma} \nabla_{\rho}^{\prime} g_{\sigma \nu} \chi_{\hat{s}}^{\nu} .
$$

After $\mathrm{H}$ is integrated out, the bosonic part of the action becomes

$$
\frac{1}{\hbar} \int_{\mathbb{R}} \mathrm{d} \hat{s}\left(\frac{1}{2} g_{\mu \nu} \partial_{\hat{s}} \phi^{\mu} \partial_{\hat{s}} \phi^{\nu}+\frac{1}{2} g^{\mu \nu} \partial_{\mu} h \partial_{\nu} h\right)+\frac{\mathrm{i}}{\hbar} \int_{\mathbb{R}} \phi^{*} a .
$$

We see that $h$ provides a potential energy. This is an analog of the superpotential $W$ in $\mathcal{N}=(2,2)$ supersymmetric sigma model.

The on-shell supersymmetry variation of $\chi$ is

$$
\delta_{\widetilde{\nabla}^{\prime}} \chi_{\hat{s}}^{\mu}=\partial_{\hat{s}} \phi^{\mu}-g^{\mu \nu} \partial_{\nu} h
$$

The connection $\widetilde{\nabla}^{\prime}$ which appears in this formula is different from $\nabla^{\prime}$ and preserves the metric: ${ }^{4}$

$$
\widetilde{\nabla}^{\prime} g=0
$$

Its coefficients are

$$
\widetilde{\Gamma}_{\nu \rho}^{\prime \mu}=\Gamma_{\nu \rho}^{\prime \mu}+\frac{1}{2} g^{\mu \sigma} \nabla_{\nu}^{\prime} g_{\sigma \rho}
$$

Note that setting $\delta_{\widetilde{\nabla}} \chi=0$ gives the equation for the gradient flow generated by $h$. This is how the relation to Morse theory arises.

The supercharge $Q$ generating the transformations (2.43)-(2.46) satisfies $Q^{2}=0$. Since the metric on $\mathbb{R}$ appears only inside the $Q$-exact part of the action, the $Q$-cohomology defines a topological theory.

Let us go back to the $\Omega$-deformed B-model. The equations of motion for the auxiliary fields are

$$
\begin{aligned}
& \mathrm{F}_{\hat{s} \hat{\theta}}^{i}=\frac{1}{1+\|V\|^{2}}\left(\bar{V}^{\hat{\theta}} \partial_{\hat{s}} \varphi^{i}+g^{i \bar{\jmath}} \partial_{\bar{\jmath}} \bar{W}+\bar{V}^{\hat{\theta}} g^{i \bar{k}} \delta \varphi^{\mu} \nabla_{\mu} g_{\bar{k} j} \rho_{\hat{s}}^{j}\right) \\
& \overline{\mathrm{F}}_{\hat{s} \hat{\theta}}^{\bar{\imath}}=\frac{1}{1+\|V\|^{2}}\left(V^{\hat{\theta}} \partial_{\hat{s}} \bar{\varphi}^{\bar{\imath}}-g^{\bar{\imath} j} \partial_{j} W-g^{\bar{\imath} k} \delta \varphi^{\mu} \nabla_{\mu} g_{k \bar{\jmath}} \mu_{\hat{s} \hat{\theta}}^{\bar{j}}\right)
\end{aligned}
$$

\footnotetext{
${ }^{4}$ The connection in the on-shell supersymmetry variation of $\chi$ can be changed to any other connection that preserves the metric as follows. Let us add to the action the $Q$-exact term $-\delta_{Q} \int_{\mathbb{R}} \mathrm{d} \hat{s} \frac{1}{2} \Upsilon_{\mu \nu \rho} \delta \phi^{\mu} \chi_{\hat{s}}^{\nu} \chi_{\hat{s}}^{\rho}$, where $\Upsilon_{\mu \nu \rho}=-\Upsilon_{\mu \rho \nu}$. Then, the equation of motion for $\mathrm{H}$ is modified, and the connection in question becomes $\widehat{\nabla}^{\prime}$ whose coefficients are $\widehat{\Gamma}_{\nu \rho}^{\prime \mu}=\widetilde{\Gamma}_{\nu \rho}^{\prime \mu}+g^{\mu \sigma} \Upsilon_{\nu \sigma \rho}$. The new connection $\widehat{\nabla}^{\prime}$ again preserves the metric (though may not be torsion-free any longer), and any connection preserving the metric can be obtained in this way. A similar modification can be made for the $\Omega$-deformed B-model for $\|V\|=1$ so that $\widetilde{\nabla}^{\prime}$ is modified to $\widehat{\nabla}^{\prime}$.

For example, $\widehat{\nabla}^{\prime}$ can be the Levi-Civita connection $\nabla^{\mathrm{LC}}$. In this case we can also take $\nabla^{\prime}=\nabla^{\mathrm{LC}}$ since $\nabla^{\mathrm{LC}}$ is torsion-free. (However, for a metric $g$ that is compatible with the complex structure but not Kähler, $\Gamma^{\mathrm{LC}}$ has mixed components and we cannot take $\nabla=\nabla^{\mathrm{LC}}$.) Another example is $\widehat{\nabla}^{\prime}=\nabla^{\mathrm{LC}}+\nabla^{\mathrm{LC}} J J$, where $J$ is an almost complex structure compatible with $g$. This connection preserves both $g$ and $J$, and is used in $[31]$.
} 
Plugging these into the supersymmetry variations of $\rho_{\hat{s}}^{i}$ and $\mu^{\bar{\imath}}$, we find

$$
\begin{gathered}
\delta_{\widetilde{\nabla}} \rho_{\hat{s}}^{i}=\frac{1}{1+\|V\|^{2}}\left(\partial_{\hat{s}} \varphi^{i}-V^{\hat{\theta}} g^{i \bar{\jmath}} \partial_{\bar{\jmath}} \bar{W}\right), \\
\delta_{\widetilde{\nabla}}\left(\bar{V}^{\hat{\theta}} \mu_{\hat{s} \hat{\theta}}^{\bar{\imath}}\right)=\frac{\|V\|^{2}}{1+\|V\|^{2}}\left(\partial_{\hat{s}} \bar{\varphi}^{\bar{\imath}}-\frac{\bar{V}}{\|V\|^{2}} g^{\bar{\imath} j} \partial_{j} W\right),
\end{gathered}
$$

where the connection $\widetilde{\nabla}$ is given by

$$
\begin{aligned}
& \widetilde{\Gamma}_{\mu j}^{i}=\Gamma_{\mu j}^{i}+\frac{\|V\|^{2}}{1+\|V\|^{2}} g^{i \bar{k}} \nabla_{\mu} g_{\bar{k} j}, \\
& \widetilde{\Gamma}_{\mu \bar{\jmath}}^{\bar{j}}=\Gamma_{\mu \bar{\jmath}}^{\bar{i}}+\frac{1}{1+\|V\|^{2}} g^{\bar{i} k} \nabla_{\mu} g_{k \bar{\jmath}} .
\end{aligned}
$$

(Adding the deformation terms (2.38) affects the equations of motion and the on-shell supersymmetry variations only for the nonzero modes.)

The quantities that appear on the right-hand sides of the above equations are complex conjugate of each other if

$$
\|V\|=1
$$

that is, if we choose

$$
\gamma_{\theta \theta}=\frac{1}{|\epsilon|^{2}}
$$

From now on we assume that this choice is made. Thus, we can write

$$
V^{\hat{\theta}}=e^{\mathrm{i} \alpha}
$$

for some $\alpha \in \mathbb{R} / 2 \pi \mathbb{Z}$. We set

$$
\hbar=\frac{|\epsilon|}{\pi}
$$

and define real functions $h, f$ by

$$
\frac{2 \pi}{\epsilon} W=\frac{1}{\hbar}(h+\mathrm{i} f),
$$

or

$$
\begin{aligned}
& h=2 \operatorname{Re}\left(e^{-\mathrm{i} \alpha} W\right), \\
& f=2 \operatorname{Im}\left(e^{-\mathrm{i} \alpha} W\right) .
\end{aligned}
$$

Comparing various formulas, especially (2.50), (2.55) and (2.56), we find that for the zero modes, the on-shell supersymmetry of the $\Omega$-deformed B-model is the same as that of supersymmetric quantum mechanics with potential $h$ and the connection $\nabla^{\prime}=\nabla$, under the following identification between the fields:

$$
\begin{aligned}
\phi & =\varphi_{0}, \\
\psi^{i} & =\iota_{V} \rho_{0}^{i}, \\
\psi^{\bar{\imath}} & =\eta_{0}^{\bar{\imath}}, \\
\star \chi^{i} & =2 e^{-\mathrm{i} \alpha} \iota_{V} \star \rho_{0}^{i}, \\
\star \chi^{\bar{\imath}} & =2 e^{-\mathrm{i} \alpha} \star \mu_{0}^{\bar{\imath}} .
\end{aligned}
$$


In components, the last two equations are $\chi_{\hat{s}}^{i}=2\left(\rho_{0}^{i}\right)_{\hat{s}}$ and $\chi_{\hat{s}}^{\bar{\imath}}=2 \bar{V}^{\hat{\theta}}\left(\mu_{0}^{\bar{\tau}}\right)_{\hat{s} \hat{\theta}}$. Note that we have $\widetilde{\nabla}=\widetilde{\nabla}^{\prime}$ for $\|V\|=1$.

Let us check that the actions also agree between the two theories. The part of the $\Omega$-deformed B-model action that contains $W$ can be written as

$$
\delta_{Q_{V}} \int_{\mathbb{R} \times \mathbb{S}^{1}} \frac{\mathrm{d} \theta}{V^{\theta}} \rho^{i} \partial_{i} W+\int_{\mathbb{R} \times \mathbb{S}^{1}} \mathrm{~d} W \wedge \frac{\mathrm{d} \theta}{V^{\theta}} .
$$

Thus, up to $Q_{V}$-exact terms, the zero mode action is given by

$$
\frac{2 \pi}{\epsilon} \int_{\mathbb{R}} \mathrm{d} W=\frac{1}{\hbar} \int_{\mathbb{R}}(\mathrm{d} h+\mathrm{i} \mathrm{d} f) .
$$

This is the action for supersymmetric quantum mechanics in the presence of the potential $h$ and the gauge field

$$
a=\mathrm{d} f,
$$

with the Planck constant $\hbar$ given by the expression (2.62).

Even more directly, one can show that the supersymmetry transformations and the actions match at the off-shell level if the auxiliary fields are identified as

$$
\begin{aligned}
& \mathrm{H}^{i}=-\mathrm{i}\left(\mathrm{d} \varphi_{0}^{i}+2 \iota_{V} \mathrm{~F}_{0}^{i}\right), \\
& \mathrm{H}^{\bar{\imath}}=+\mathrm{i}\left(\mathrm{d} \bar{\varphi}_{0}^{\bar{\imath}}+2 \iota \bar{V}_{\bar{V}} \overline{\mathrm{F}}_{0}^{\bar{\imath}}\right) .
\end{aligned}
$$

Thus, we have shown that the circle reduction of the $\Omega$-deformed B-model is the topological twist of supersymmetric quantum mechanics.

\subsection{Cigar reduction of the $\Omega$-deformed B-model}

Having understood the circle reduction of the $\Omega$-deformed B-model, let us now consider the cigar reduction. We take $\Sigma$ to be a cigar $\mathbb{D}$, consisting of a finite cylinder capped at one end. As before, we endow $\mathbb{D}$ with a rotation invariant metric, with $\gamma_{\theta \theta}(s)=1 /|\epsilon|^{2}$ on the cylinder part. The boundary of $\mathbb{D}$ is located at $s=\ell$, and the flat cylinder region continues till a small value of $s$, where the cylinder is curved abruptly inward to cover the hole. The tip of the cigar is at $s=0$.

Reduction on the circle fibers of $\mathbb{D}$ produces supersymmetric quantum mechanics on the interval $\mathbb{I}=[0, \ell]$. The space has two boundaries, one coming from the boundary circle of $\mathbb{D}$ and the other from the region near the tip. We wish to understand what happens at these points.

At $s=\ell$, the boundary conditions for supersymmetric quantum mechanics is simply the reduction of the boundary conditions chosen on $\partial \mathbb{D}$ in the $\Omega$-deformed B-model. We take the latter boundary conditions to be $Q_{V}$-invariant. Then, the former are $Q$-invariant.

In general, in the $\Omega$-deformed B-model on $\Sigma$, one imposes a brane-type boundary condition such that the bosonic field maps the boundary to a chosen submanifold $L \subset X$ :

$$
\varphi(\partial \Sigma) \subset L
$$


If $\Sigma$ has more than one boundary components, one picks such a submanifold for each component of $\partial \Sigma$.

Furthermore, one may turn on a boundary superpotential $W_{0}$, a locally constant function on $L$. This introduces an additional term to the boundary part of the action, which is now given by

$$
-\int_{\partial \Sigma}\left(W-W_{0}\right) \frac{\mathrm{d} \theta}{V^{\theta}} .
$$

Finally, for the path integral to not diverge, one must choose $L$ in such a way that the integrand of the boundary action is bounded from above on $L$ :

$$
\left.\operatorname{Re}\left(\frac{W-W_{0}}{V^{\theta}}\right)\right|_{L}<+\infty
$$

The rest of the boundary conditions are as follows. Since the boundary conditions should preserve $Q_{V}$, the supersymmetry variation of the condition (2.76) must also hold:

$$
\iota_{V} \rho^{i} \partial_{i}+\eta^{\bar{\imath}} \partial_{\bar{\imath}} \in T_{\varphi} L \otimes \mathbb{C} .
$$

Requiring that no boundary term arises when we vary fermions in the action, we find

$$
\iota_{V} \star \rho^{i} \partial_{i}+\star \mu^{\bar{\imath}} \partial_{\bar{\imath}} \in N_{\varphi} L \otimes \mathbb{C}
$$

on the boundary, where $N_{\varphi} L$ is the normal space to $L$ at $\varphi$. The supersymmetry variation of this condition gives

$$
\left(\partial_{\hat{s}} \varphi^{\mu}-g^{\mu \nu} \partial_{\nu} h\right) \partial_{\mu} \in N_{\varphi} L .
$$

Here we used the fact that $\widetilde{\nabla}$ is a metric connection and hence the covariant variation $\delta_{\widetilde{\nabla}}$ maps a normal vector to a normal vector.

The above boundary conditions reduce to the following boundary conditions in supersymmetric quantum mechanics:

$$
\begin{aligned}
\phi & \in L, \\
\psi & \in T_{\phi} L \otimes \mathbb{C}, \\
\star \chi & \in N_{\phi} L \otimes \mathbb{C}, \\
\star \mathrm{d} \phi-\phi^{*}\left(g^{-1} \mathrm{~d} h\right) & \in N_{\phi} L .
\end{aligned}
$$

The boundary conditions at $s=0$ have a different flavor as they comes from the tip of the cigar, which is not a boundary in two dimensions. Since $\varphi$ is unconstrained at the tip, $\phi$ can also take any values in $X$. At the tip $V$ vanishes, so we have

$$
\psi^{i}=\chi^{i}=0
$$

at $s=0$. From the point of view of the $\Omega$-deformed B-model, the origin of these boundary conditions is the positive curvature near the tip of the cigar. The curvature makes the one-form $\rho$ massive, thereby eliminating it from the effective description.

Taking the supersymmetry variations of these equations, we find

$$
\partial_{\hat{s}} \phi^{\mu}-g^{\mu \nu} \partial_{\nu} h=0 \text {. }
$$


As we will see, the path integral for supersymmetric quantum mechanics may be localized to the solutions of this equation. Hence, the boundary condition at $s=0$ does not really constrain $\phi$.

Let us calculate the action. The tip of $\mathbb{D}$ is a special point, so we first excise a small disk $\mathbb{D}_{0}$ around it. On $\mathbb{D} \backslash \mathbb{D}_{0}$, the calculation is the same as in the case of $\mathbb{R} \times \mathbb{S}^{1}$, except this time we want to take boundary terms into account. Up to $Q_{V}$-exact terms, the action is

$$
\frac{1}{\epsilon} \int_{\mathbb{D} \backslash \mathbb{D}_{0}} \mathrm{~d} W \wedge \mathrm{d} \theta-\frac{1}{\epsilon} \int_{\partial\left(\mathbb{D} \backslash \mathbb{D}_{0}\right)} W \mathrm{~d} \theta+\frac{1}{\epsilon} \int_{\partial \mathbb{D}} W_{0} \mathrm{~d} \theta=\frac{1}{\epsilon} \int_{\partial \mathbb{D}} W_{0} \mathrm{~d} \theta
$$

To this we add the contribution from $\mathbb{D}_{0}$. We can evaluate it by taking the radius of $\mathbb{D}_{0}$ to zero. The bulk integral then vanishes since the Lagrangian has no singularity at the tip, but the boundary integral remains and gives

$$
-\frac{2 \pi}{\epsilon} W(0) \text {. }
$$

Therefore, the action for supersymmetric quantum mechanics on $\mathbb{I}$ is, up to $Q$-exact terms,

$$
\frac{2 \pi}{\epsilon}\left(W_{0}-W(0)\right)=\frac{1}{\hbar}\left(h_{0}+\mathrm{i} f_{0}-h(0)-\mathrm{i} f(0)\right),
$$

where we have defined the constants $h_{0}, f_{0}$ by $2 \pi W_{0} / \epsilon=h_{0}+\mathrm{i} f_{0}$. This is nothing but

$$
\frac{1}{\hbar} \int_{\mathbb{I}}(\mathrm{d} h+\mathrm{i} \mathrm{d} f)+\frac{1}{\hbar}\left(h_{0}+\mathrm{i} f_{0}-h(\ell)-\mathrm{i} f(\ell)\right),
$$

namely the non- $Q$-exact part of the action (2.47) plus boundary terms at $s=\ell$ which come from the boundary term (2.77) in the $\Omega$-deformed B-model. Note that the boundary conditions at $s=0$ make this expression $Q$-invariant since $h+\mathrm{i} f$ is a holomorphic function.

\subsection{Reduction to zero-dimensional sigma model}

In [2], it was shown that if $X$ is Kähler and the support $L$ of the brane on $\partial \mathbb{D}$ is a Lagrangian submanifold of $X$, the path integral for the $\Omega$-deformed B-model on $\mathbb{D}$ is equivalent to the path integral for a zero-dimensional bosonic sigma model. Here we derive a slightly more general result, applicable to the case in which $X$ is not necessarily Kähler, starting from the description in terms of supersymmetric quantum mechanics.

Let us rescale the metric of $\mathbb{I}$ by a very small factor. Then, the kinetic term for $\phi$ in the action (2.49) becomes very large, whereas the potential term becomes very small. The path integral thus localizes to constant maps. By the boundary condition at $s=\ell$, these maps must be valued in $L$. The boundary condition on $\phi$ at $s=0$ reduces to $\partial_{s} \phi=0$ and is satisfied by constant maps.

Now, suppose that $L$ is middle-dimensional and the pullback of the two-form $\omega=g I$ by the inclusion map $i_{L}: L \hookrightarrow X$ vanishes:

$$
i_{L}^{*} \omega=0
$$

If $g$ is a Kähler metric (that is, if $\mathrm{d} \omega=0$ ), then $\omega$ defines a symplectic structure on $X$ and this condition means that $L$ is a Lagrangian submanifold. In general, $\omega$ is only an almost 
symplectic structure, but we will still call such a submanifold $L$ Lagrangian, and refer to the corresponding boundary conditions at $s=\ell$ as a Lagrangian brane with support $L$.

When $L$ is Lagrangian, the fermionic fields have no zero modes, as can be shown as follows. The boundary conditions (2.86) at $s=0$ kill the constant modes of $\psi^{i}$ and $\chi^{i}$. Then, by the boundary conditions (2.83) and (2.84) at $s=\ell$, the constant modes of $\psi^{\bar{\imath}} \partial_{\bar{\imath}}$ and $\chi_{\hat{s}}^{\bar{\tau}} \partial_{\bar{\imath}}$ must belong to $T_{\phi} L \otimes \mathbb{C}$ and $N_{\phi} L \otimes \mathbb{C}$, respectively. Since $i_{L}^{*} \omega=0$, we have $g(v, I w)=0$ for any $v, w \in T_{\phi} L$ and therefore $I\left(T_{\phi} L\right) \subset N_{\phi} L$. As $L$ is middledimensional, $I$ exchanges $T_{\phi} L$ and $N_{\phi} L$. Thus, we have $I\left(\psi^{\bar{\imath}} \partial_{\bar{\imath}}\right)=-\mathrm{i} \psi^{\bar{\imath}} \partial_{\bar{\imath}} \in N_{\phi} L \otimes \mathbb{C}$ and $-\mathrm{i} \chi_{\hat{s}}^{\bar{\imath}} \partial_{\bar{\imath}} \in T_{\phi} L \otimes \mathbb{C}$. It follows that both $\psi^{\bar{\imath}} \partial_{\bar{\imath}}$ and $\chi_{\hat{s}}^{\bar{\imath}} \partial_{\bar{\imath}}$ belong to $T_{\phi} L \otimes \mathbb{C}$ and $N_{\phi} L \otimes \mathbb{C}$ simultaneously, hence they are actually zero.

Finally, the integration over the fluctuations of the bosonic and fermionic fields produce one-loop determinants that cancel each other out. This is because of supersymmetry and the fact that the fluctuations of $\phi, \psi$ and $I \star \chi$ all obey the same boundary conditions: they vanish at $s=0$ and belong to $T_{\phi} L \otimes \mathbb{C}$ at $s=\ell$.

With the fluctuations integrated out, the only remaining integration variables is the zero mode of $\phi$. We conclude that the path integral for supersymmetric quantum mechanics on $\mathbb{I}$ reduces to the bosonic integral

$$
\int_{L} \operatorname{vol}_{L} \exp \left(\frac{2 \pi}{\epsilon}\left(W-W_{0}\right)\right)=\int_{L} \operatorname{vol}_{L} \exp \left(\frac{1}{\hbar}\left(h+\mathrm{i} f-h_{0}-\mathrm{i} f_{0}\right)\right),
$$

where $\operatorname{vol}_{L}$ is a volume form on $L$. This is the path integral for a zero-dimensional sigma model with target $L$.

\subsection{Multivalued superpotentials}

There is an important generalization of the above story that will be relevant for many applications. A crucial observation is the following: if $\Sigma$ has no boundary, $W$ does not have to be single-valued. Indeed, all we need to write down the action for the $\Omega$-deformed B-model is $\mathrm{d} W$, not $W$ itself.

If $\Sigma$ does have a boundary, $W$ may still be multivalued but one must make sense of the boundary term (2.77), in which $W$ appears directly. Hence, in the presence of boundary, one must define $W$ on $L$. The definition of $W$ needs to be given only modulo i $\in \mathbb{Z}$ since the action appears as $e^{-S}$ in the path integral.

Given a 1-form $\mathrm{d} W$ on $X$, one may try to define $W$ on $L$ as follows. In each pathconnected component $L_{a}$ of $L$, one picks a reference point $p_{a}$ and declares $W\left(p_{a}\right)=0$. Then, at any point $p$ of $L_{a}$, one defines $W(p)$ by choosing a path $P$ from $p_{a}$ to $p$ and setting

$$
W(p)-W\left(p_{a}\right)=\int_{P} \mathrm{~d} W .
$$

The resulting function is generally multivalued since this definition depends on the choice of the path. This is not a problem if $\mathrm{d} W$ satisfies

$$
\left[i_{L}^{*} \mathrm{~d} W\right] \in \mathrm{i} \epsilon H^{1}(L ; \mathbb{Z}),
$$

for then $W$ is well-defined modulo $i \in \mathbb{Z}$. Therefore, this construction provides a good definition of $W$ on $L$, as long as the above quantization condition is obeyed. Note that the 
real part of the quantization condition says that $\mathrm{d} h$ is trivial in the cohomology, so $h$ is single-valued on $L$.

If $W$ does not exist as a well-defined function modulo $i \in \mathbb{Z}$ on all of $X$, the expression (2.90) of the action is not valid because the meaning of $W(0)$ is ambiguous. If either the real or imaginary part of $\mathrm{d} W / \epsilon$ obeys the quantization condition on the entire $X$, then the Stokes theorem can be applied to that part in the expression (2.91).

At any rate, the localization formula (2.93) remains valid since the path integral localizes to $L$, where $W$ is well-defined modulo $i \in \mathbb{Z}$.

The situation that will arise when we discuss the relation between the $\Omega$-deformed Rozansky-Witten theory and the A-model is that the imaginary part of $\mathrm{d} W / \epsilon$ obeys the quantization condition on $X$. In terms of the flat connection $a=2 \pi \hbar \operatorname{Im}(\mathrm{d} W / \epsilon)$, the quantization condition is

$$
[a] \in 2 \pi \hbar H^{1}(X ; \mathbb{Z}) .
$$

Moreover, we will find that for the condition (2.78) to be satisfied, we must have $h-h_{0}=0$ on $L$. This condition can be satisfied by an appropriate choice of $h_{0}$ if and only if $h$ is locally constant on $L$ :

$$
i_{L}^{*}(\mathrm{~d} h)=0 .
$$

In this situation, we can define the function $f$ modulo $2 \pi \hbar$ on $X$, and the action can be written as

$$
\frac{1}{\hbar} \int_{\mathbb{I}} \mathrm{d} h+\frac{\mathrm{i}}{\hbar}\left(f_{0}-f(0)\right),
$$

up to $Q$-exact terms. We may think of this expression as the action for supersymmetric quantum mechanics with potential $h$, with $f(0)$ and $f_{0}$ being zero-form boundary gauge fields turned on at $s=0$ and $s=\ell$, respectively.

\subsection{Lagrangian branes from the gradient flow}

If $M$ is Kähler, there is a way to construct good Lagrangian submanifolds, which at the same time provides a definition of $W$ without help of any quantization condition $[9,30,50]$. These Lagrangian branes are the boundary conditions produced at $s=0$ by supersymmetric quantum mechanics on the half-line $[0,+\infty)$.

Consider supersymmetric quantum mechanics on $[0,+\infty)$, and rescale the $Q$-exact part of the action (2.47) by a large factor $u$. Then, the bosonic part of the action becomes

$$
\frac{u}{\hbar} \int_{[0,+\infty)} \mathrm{d} \hat{s} \frac{1}{2} g_{\mu \nu}\left(\partial_{\hat{s}} \phi^{\mu}-g^{\mu \rho} \partial_{\rho} h\right)\left(\partial_{\hat{s}} \phi^{\nu}-g^{\nu \sigma} \partial_{\sigma} h\right)+\frac{1}{\hbar} \int_{[0,+\infty)}\left(\mathrm{d} h+\mathrm{i} \phi^{*} a\right) .
$$

The real part of the action remains positive semidefinite, so this is a valid deformation.

We see that in the limit $u \rightarrow \infty$, the path integral localizes to the solutions of the gradient flow equation

$$
\partial_{\hat{s}} \phi^{\mu}-g^{\mu \nu} \partial_{\nu} h=0 .
$$

For a solution $\hat{\phi}_{p}$ with initial condition $\hat{\phi}_{p}(0)=p$, the real part of the bosonic action evaluates to $\left(h\left(\hat{\phi}_{p}(+\infty)\right)-h(p)\right) / \hbar$. Since $h$ is monotonically increasing along the flow, the contribution to the path integral from $\hat{\phi}_{p}$ vanishes unless $\hat{\phi}_{p}(+\infty)$ is a critical point of $h$. 
For this reason, let us choose the boundary condition at $s=+\infty$ in such a way that $\phi(+\infty)$ lies in a submanifold $L_{\infty}$ of the critical locus Crit $(h)$ of $h$, and define $L$ to be the set of all points $p$ such that the gradient flow $\hat{\phi}_{p}$ reaches $L_{\infty}$ at $s=+\infty$ :

$$
L=\left\{p \in X \mid \hat{\phi}_{p}(+\infty) \in L_{\infty}\right\}
$$

For $L$ thus constructed, the condition (2.78) is satisfied since $\left.h\right|_{L}$ is bounded above by the constant $\left.h\right|_{L_{\infty}}$.

For $L$ to be a Lagrangian submanifold, $L_{\infty}$ must obey an additional condition. Since $h=2 \operatorname{Re}\left(e^{-\mathrm{i} \alpha} W\right)$, we have $\operatorname{Crit}(h)=\operatorname{Crit}(W)$. The critical locus is therefore a complex submanifold of $X$ and itself Kähler. A necessary and sufficient condition is that $L_{\infty}$ is a Lagrangian submanifold of $\operatorname{Crit}(W)$.

To see that this is sufficient, note that the gradient flow generated by $h$ coincides with the Hamiltonian flow generated by $f$ (if $f$ is globally defined, to be precise) because $\iota_{\partial_{\hat{s}} \phi} \omega=g^{\mu \nu} \partial_{\nu} h \omega_{\mu \rho} \mathrm{d} \phi^{\rho}=\partial_{\nu} h I^{\nu}{ }_{\rho} \mathrm{d} \phi^{\rho}=-\mathrm{d} f$. It follows that $\omega$ is preserved along the flow: $\mathcal{L}_{\partial_{\hat{s}} \phi} \omega=\left(\mathrm{d} \iota_{\partial_{\hat{s}} \phi}+\iota_{\partial_{\hat{s}} \phi} \mathrm{d}\right) \omega=0 .{ }^{5}$ Since $\omega$ is preserved, we can evaluate $\omega(v, w)$ for any $v$, $w \in T_{p} L$ by pushing the vectors forward along the flow $\hat{\phi}:[0, \infty) \times X \rightarrow X$. Following the flow, we eventually reach a fixed point, where we have $\omega\left(\hat{\phi}(+\infty)_{*} v, \hat{\phi}(+\infty)_{*} w\right)=0$ if $L_{\infty}$ is Lagrangian. Hence, $\omega(v, w)=0$. That $L$ is middle-dimensional follows from the fact that the Hessian of $h$ has the same number of positive and negative eigenvalues because $h$ is the real part of a holomorphic function.

The necessity of the above condition is clear since $L_{\infty}$ is itself contained in $L$, and $L_{\infty}$ must be a middle-dimensional submanifold of $\operatorname{Crit}(W)$ in order for $L$ to be middledimensional in $X$.

On a Lagrangian brane constructed in this way, one can define $h$ at $p \in L$ in terms of the value of $h$ at the fixed point $\hat{\phi}_{p}(+\infty) \in L_{\infty}$ by integrating the gradient flow equation. The value of $f$ is constant along the flow. Since $W$ is constant on $L_{\infty}$, defining $W$ on $L$ amounts to choosing a single complex number as the value of $W$ on each connected component of $L_{\infty}$. This constant may be absorbed in $W_{0}$, so does not introduce further ambiguity.

\section{Rozansky-Witten theory, A-model and quantum mechanics}

As a first application of the results obtained above, in this section we establish the equivalences between the $\Omega$-deformed Rozansky-Witten theory on $\mathbb{D} \times \mathbb{M}_{1}$, the A-model on $\mathbb{I} \times \mathbb{M}_{1}$ and quantum mechanics on $\mathbb{M}_{1}$. These equivalences connect two approaches to quantization, namely brane quantization [1] and quantization by the $\Omega$-deformation [2].

\subsection{Rozansky-Witten theory}

Rozansky-Witten theory [39] is a three-dimensional topological field theory, and may be obtained by topological twist from $\mathcal{N}=4$ supersymmetric sigma model, for which the

\footnotetext{
${ }^{5}$ This is where the Kähler condition $\mathrm{d} \omega=0$ is necessary. In the almost symplectic case, the argument goes through provided $\iota_{\partial_{\hat{s}} \phi} \mathrm{d} \omega=0$.
} 
target space must be hyperkähler. However, just as the B-model can be constructed for nonKähler target spaces, the target space of Rozansky-Witten theory can be more generally a complex symplectic manifold.

A complex symplectic manifold $Y$ is a complex manifold endowed with a holomorphic symplectic form $\Omega$, a closed nondegenerate $(2,0)$-form. The real and imaginary parts of $\Omega$ are real symplectic forms $\omega_{J}$ and $\omega_{K}$ :

$$
\Omega=\omega_{J}+\mathrm{i} \omega_{K} .
$$

We denote the complex structure of $Y$ by $I$.

There exists an almost complex structure $K$ such that $\omega_{K}$ is compatible with $K$ and $-\omega_{K} K$ is positive. Moreover, one can choose $K$ in such a way that $I K=-K I$. Then, $J=K I$ is also an almost complex structure, and $\omega_{J}$ is compatible with $J$ since $J^{t} \omega_{J} J=$ $-J^{t} \omega_{J} I K=J^{t} \omega_{K} K=-J^{t} K^{t} \omega_{K}=I^{t} \omega_{K}=\omega_{J}$. The three almost complex structures $I$, $J, K$ satisfy the quaternion relations

$$
I^{2}=J^{2}=K^{2}=I J K=-1 .
$$

There is a metric $g$ compatible with both $\left(J, \omega_{J}\right)$ and $\left(K, \omega_{K}\right)$ :

$$
g=-\omega_{J} J=\omega_{J} I K=-\omega_{K} K .
$$

This metric is also compatible with $I$ since $I^{t} g I=-I^{t} \omega_{J} J I=-\omega_{K} K=g$, and

$$
\omega_{I}=g I
$$

is a $(1,1)$-form with respect to $I$. In general, $\omega_{I}$ is not closed and $g$ is not Kähler. In fact, $Y$ is hyperkähler if and only if $\omega_{I}$ is closed [51]. A hyperkähler manifold has three integrable complex structures $I, J, K$, obeying the quaternion relations, and a metric $g$ that is Kähler with respect to each of $I, J, K$.

The fields of Rozansky-Witten theory with complex symplectic target $Y$, placed on a three-manifold $\mathbb{M}_{3}$, are

$$
\begin{aligned}
\varphi & \in \operatorname{Map}\left(\mathbb{M}_{3}, Y\right), \\
\eta & \in \Pi \Omega^{0}\left(\mathbb{M}_{3}, \varphi^{*} T^{0,1} Y\right), \\
\rho & \in \Pi \Omega^{1}\left(\mathbb{M}_{3}, \varphi^{*} T^{1,0} Y\right) .
\end{aligned}
$$

The theory has supersymmetry which transform the fields as

$$
\begin{aligned}
\delta \varphi^{i} & =0, \\
\delta \rho^{i} & =\mathrm{d} \varphi^{i}, \\
\delta \varphi^{\bar{\imath}} & =\eta^{\bar{\imath}}, \\
\delta \eta^{\bar{\imath}} & =0 .
\end{aligned}
$$

The supercharge $Q_{0}$ for these transformations satisfies $Q_{0}^{2}=0$, and one considers the $Q_{0}$-cohomology. 
To write down the action for Rozansky-Witten theory, one must choose a metric $g$ and a torsion-free connection $\nabla$ on $Y$, both compatible with $I$, and a metric on $\mathbb{M}_{3}$. The action is given by

$$
\begin{array}{r}
S_{\mathrm{RW}}=\delta_{Q_{0}} \int_{\mathbb{M}_{3}} g_{i \bar{\jmath}} \rho^{i} \wedge \star \mathrm{d} \bar{\varphi}^{\bar{\jmath}}-\frac{\mathrm{i}}{4} \int_{\mathbb{M}_{3}}\left(\Omega_{i j} \rho^{i} \wedge \mathrm{d} \nabla \rho^{j}+\frac{1}{3} \nabla_{k} \Omega_{i j} \mathrm{~d} \varphi^{i} \wedge \rho^{j} \wedge \rho^{k}\right. \\
\left.-\frac{1}{3} \Omega_{i j}\left(R_{\nabla}\right)^{j}{ }_{k l \bar{m}} \rho^{i} \wedge \rho^{k} \wedge \rho^{l} \eta^{\bar{m}}\right) .
\end{array}
$$

The metric $g$ appears only in the $Q_{0}$-exact part of the action, so the theory does not depend on the choice of $g$. It turns out that the non- $Q_{0}$-exact part changes by a $Q_{0}$-exact term if $\nabla$ is replaced by another connection. Thus, the theory is also independent of the choice of $\nabla$.

\subsection{B-model formulation of Rozansky-Witten theory}

In order to apply the results from the previous section, we need to describe RozanskyWitten theory as a B-model. This is possible when the spacetime is of the form $\mathbb{M}_{3}=$ $\Sigma \times \mathbb{M}_{1}$, where $\Sigma$ is a two-manifold and $\mathbb{M}_{1}$ is a one-manifold. We denote a coordinate on $\mathbb{M}_{1}$ by $t$.

In this case, the bosonic field $\varphi: \Sigma \times \mathbb{M}_{1} \rightarrow Y$ of Rozansky-Witten theory may be identified with a map from $\Sigma$ to $\operatorname{Map}\left(\mathbb{M}_{1}, Y\right)$. As such, it can be the bosonic field of the B-model on $\Sigma$ with target space

$$
X=\operatorname{Map}\left(\mathbb{M}_{1}, Y\right)
$$

A vector field $v$ on $X$, evaluated at $\varphi \in X$, is a section of the bundle $\varphi^{*} T Y$ over $\mathbb{M}_{1}$ and can be written locally as $v(t)=v^{\mu}(t) \partial_{\mu}$. Tensor fields on $X$ can be expressed likewise. A complex structure $I$ and a compatible torsion-free connection $\nabla$ on $X$ are naturally induced from those on $Y$ : for a vector $v$ and a tensor $w$ on $X$,

$$
\begin{aligned}
(I v)(t) & =I v(t), \\
\left(\nabla_{v} w\right)(t) & =\nabla_{v(t)} w(t) .
\end{aligned}
$$

Similarly, given a metric on $\mathbb{M}_{1}$, a metric on $X$ is induced from that on $Y$ : the inner product between $v, w \in T_{\varphi} X$ is given by

$$
g(v, w)=\int_{\mathbb{M}_{1}} \mathrm{~d} t g(v(t), w(t)),
$$

where we have chosen $t$ in such a way that the metric on $\mathbb{M}_{1}$ is $\mathrm{d} t^{2}$.

As can be seen from the above formula for the metric, one may think of the coordinate $t$ as a "continuous index." In diffeomorphism invariant expressions, $t$ should be integrated over, just as the indices $i, j, \ldots$ and $\bar{\imath}, \bar{\jmath}, \ldots$ are to be summed over.

Keeping this in mind, we can immediately write down the standard B-model action (the formula (2.34) with $V=0$ ) for the target space $X$ :

$$
S_{\mathrm{B}, \mathrm{C}}=\delta_{Q_{0}} \int_{\Sigma} \int_{\mathbb{M}_{1}} \mathrm{~d} t g_{i \bar{\jmath}}\left(\rho^{i} \wedge \star_{\Sigma} \mathrm{d}_{\Sigma} \bar{\varphi}^{\bar{\jmath}}+\mathrm{F}^{i} \wedge \star_{\Sigma} \mu^{\bar{\jmath}}\right) .
$$


The Hodge star and the exterior derivative in this action are defined with respect to $\Sigma$ since the spacetime of the B-model is $\Sigma$ and does not include $\mathbb{M}_{1}$.

Compared to the Rozansky-Witten action (3.12), many terms are missing from this action. Crucially, all kinetic terms that involve derivatives along $\mathbb{M}_{1}$ cannot be present here because, again, $\mathbb{M}_{1}$ is not part of the spacetime. These missing terms must come from a superpotential.

A superpotential with the required property is constructed as follows. Since the holomorphic symplectic form $\Omega$ is closed, locally we can write it as

$$
\Omega=\mathrm{d} \Lambda
$$

for some holomorphic one-form $\Lambda$. Then, for $\varphi \in X$, we set

$$
W(\varphi)=\frac{\mathrm{i}}{2} \int_{\mathbb{M}_{1}} \varphi^{*} \Lambda
$$

(The precise definition of $W$ involves the integral of $\Omega$ over a cobordism from a reference copy of $\mathbb{M}_{1}$ inside $Y$ to $\varphi\left(\mathbb{M}_{1}\right)$, as explained in section 2.5.) In terms of local holomorphic Darboux coordinates $\left(P_{m}, Q^{m}\right)$ such that $\Omega=\mathrm{d} P_{m} \wedge \mathrm{d} Q^{m}$, we may write $\Lambda=P_{m} \mathrm{~d} Q^{m}$.

In the present setup, the derivative $\partial_{\mu}$ that appears in the superpotential action (2.35) should be replaced by the functional derivative $\delta / \delta \varphi^{\mu}(t)$. For the above superpotential, the functional derivative of $W$ is

$$
\frac{\delta W}{\delta \varphi^{i}}=\frac{\mathrm{i}}{2} \Omega_{i j} \partial_{t} \varphi^{j}
$$

The equations of motion for the auxiliary fields are

$$
\begin{aligned}
\star_{\Sigma} \mathrm{F}^{i} & =-\frac{\mathrm{i}}{2} g^{i \bar{\jmath}} \bar{\Omega}_{\bar{\jmath} \bar{k}} \partial_{t} \bar{\varphi}^{\bar{k}}, \\
\star_{\Sigma} \overline{\mathrm{F}}^{\bar{\imath}} & =-\frac{\mathrm{i}}{2} g^{\bar{\imath} j} \Omega_{j k} \partial_{t} \varphi^{k}-g^{\bar{\imath} j} \eta^{\bar{k}} \nabla_{\bar{k}} g_{j \bar{l}} \star_{\Sigma} \mu^{\bar{l}},
\end{aligned}
$$

and we have the on-shell supersymmetry variation

$$
\delta_{\nabla}\left(\star_{\Sigma} \mu^{\bar{\imath}}\right)=-\frac{\mathrm{i}}{2} g^{\bar{i} j} \Omega_{j k} \partial_{t} \varphi^{k}-g^{\bar{\imath} j} \eta^{\bar{k}} \nabla_{\bar{k}} g_{j \bar{l}} \star_{\Sigma} \mu^{\bar{l}} .
$$

Comparing the last equation with the supersymmetry variations (3.8), (3.9) and (3.10) in Rozansky-Witten theory, we deduce the identification

$$
\star \Sigma \mu^{\bar{\imath}}=-\frac{\mathrm{i}}{2} g^{\bar{\imath} j} \Omega_{j k} \rho_{t}^{k} .
$$

The rest of the fermions, $\eta$ and $\rho$ in the B-model, are identified with $\eta$ and the components of $\rho$ along $\Sigma$ in Rozansky-Witten theory.

Under this identification of the fields, we can show that the action for the B-model matches that for Rozansky-Witten theory. To show this, we take the metric $g$ to be the one given by the relations (3.3). This metric satisfies $\bar{\Omega}^{t} g^{-1} \Omega=2 g(1-\mathrm{i} I)=2(1+\mathrm{i} I)^{t} g$, or $\bar{\Omega}_{\bar{k} \bar{\imath}} g^{\bar{k} l} \Omega_{l j}=4 g_{\bar{\imath} j}$. Furthermore, we choose $\nabla$ to be a connection that preserves $\Omega$. Such a torsion-free connection compatible with $I$ exists [52]. 
It is clear that the $Q_{0}$-exact terms

$$
\delta_{Q_{0}} \int_{\Sigma \times \mathbb{M}_{1}} \mathrm{~d} t \wedge\left(g_{i \bar{\jmath}} \rho^{i} \wedge \star_{\Sigma} \mathrm{d}_{\Sigma} \bar{\varphi}^{\bar{\jmath}}+\frac{\mathrm{i}}{2} \mu^{\bar{\imath}} \bar{\Omega}_{\bar{\imath} \bar{\jmath}} \partial_{t} \bar{\varphi}^{\bar{\jmath}}\right)
$$

contained in the B-model action, reproduce the $Q_{0}$-exact part of the Rozansky-Witten action (3.12). The remaining terms in the B-model action are

$$
\begin{aligned}
\int_{\Sigma \times \mathbb{M}_{1}} \mathrm{~d} t \wedge\left(g_{i \bar{\jmath}} \mathrm{F}^{i} \wedge_{\Sigma} \star \overline{\mathrm{F}}^{\bar{\jmath}}+\eta^{\bar{k}} \nabla_{\bar{k}} g_{i \bar{\jmath}} \mathrm{F}^{i} \wedge \star_{\Sigma} \mu^{\bar{\jmath}}+\frac{\mathrm{i}}{2} \mathrm{~F}^{i} \Omega_{i j} \partial_{t} \varphi^{j}\right. \\
\left.\quad+g_{i \bar{\jmath}}\left(\mathrm{d}_{\nabla, \Sigma} \rho^{i}-\frac{1}{2}\left(R_{\nabla}\right)^{i}{ }_{j k \bar{l}} \bar{l}^{\bar{l}} \rho^{j} \wedge \rho^{k}\right) \wedge \star \Sigma \mu^{\bar{\jmath}}-\frac{\mathrm{i}}{4} \Omega_{i j} \rho^{i} \wedge \nabla_{t} \rho^{j}\right),
\end{aligned}
$$

where $\mathrm{d}_{\nabla, \Sigma}$ is the operator $\mathrm{d}_{\nabla}$ restricted to $\Sigma$ and $\mathrm{d}_{\nabla, \mathbb{M}_{1}}=\mathrm{d} t \wedge \nabla_{t}$. The terms proportional to $\mathrm{F}$ cancel by the equation of motion for $\overline{\mathrm{F}}$. Using the first Bianchi identity $\left(R_{\nabla}\right)^{\mu}{ }_{\nu \rho \sigma}+$ $\left(R_{\nabla}\right)_{\rho \sigma \nu}^{\mu}+\left(R_{\nabla}\right)_{\sigma \nu \rho}^{\mu}=0$, which holds for any torsion-free connection, and the identity $\Omega_{i k}\left(R_{\nabla}\right)^{k}{ }_{j l \bar{m}}+\Omega_{k j}\left(R_{\nabla}\right)^{k}{ }_{j l \bar{m}}=0$, which follows from $\nabla \Omega=0$, one can check that the other terms reproduce the non- $Q_{0}$-exact part of the Rozansky-Witten action.

\section{$3.3 \Omega$-deformed Rozansky-Witten theory and quantum mechanics}

Let us take $\Sigma$ to be a disk $\mathbb{D}$ with a rotation invariant metric. Being a B-model, RozanskyWitten theory on $\mathbb{D} \times \mathbb{M}_{1}$ can be subjected to the $\Omega$-deformation described in section 2 . As we now show, this $\Omega$-deformed Rozansky-Witten theory is equivalent to quantum mechanics with vanishing Hamiltonian.

The support $L$ of the brane on $\partial \mathbb{D}$ must be a "middle-dimensional" submanifold of $X$ such that the symplectic form $\omega$ on $X$, induced from $\omega_{I}$ by

$$
\omega(v, w)=\int_{\mathbb{M}_{1}} \mathrm{~d} t \omega_{I}(v(t), w(t))
$$

vanishes when pulled back to $L$. In the present infinite-dimensional setup, this condition should be interpreted as the requirement that the action of the complex structure exchanges the tangent bundle and the normal bundle of $L$. To impose this condition in a local fashion, we take a Lagrangian submanifold $M \subset Y$ and set

$$
L=\operatorname{Map}\left(\mathbb{M}_{1}, M\right) .
$$

(Recall that we have defined a Lagrangian submanifold of an almost symplectic manifold to be a middle-dimensional submanifold on which the pullback of the almost symplectic form vanishes.)

The boundary superpotential is given by the integral of a complex gauge field $\Lambda_{0}$ on $M$ :

$$
W_{0}(\varphi)=\frac{\mathrm{i}}{2} \int_{\mathbb{M}_{1}} \varphi^{*} \Lambda_{0}
$$

For $W_{0}$ to be locally constant on $L$, the boundary gauge field must be flat:

$$
\mathrm{d} \Lambda_{0}=0 \text {. }
$$


We should also make sure that $h-h_{0}$ is bounded on $L$. This condition actually requires $h-h_{0}=0$ on $L$ [9]. To see this, let us consider the case $\mathbb{M}_{1}=\mathbb{S}^{1}$. Then, for any point $\varphi \in L$, the map $\varphi_{n}$ defined by $\varphi_{n}(t)=\varphi(n t)$ with $n \in \mathbb{Z}$ is also a point in $L$. Since $h\left(\varphi_{n}\right)-h_{0}\left(\varphi_{n}\right)=n\left(h(\varphi)-h_{0}(\varphi)\right)$, if $h-h_{0}$ is nonzero somewhere in $L$, it is unbounded.

For this condition to be satisfied, $\operatorname{Im}\left(e^{-\mathrm{i} \alpha} i_{M}^{*} \Lambda / \epsilon\right)$ must be a flat connection so that we can set $\operatorname{Im}\left(e^{-\mathrm{i} \alpha} \Lambda_{0} / \epsilon\right)=-\operatorname{Im}\left(e^{-\mathrm{i} \alpha} i_{M}^{*} \Lambda / \epsilon\right)$. Equivalently, we must have $\operatorname{Im}\left(e^{-\mathrm{i} \alpha} i_{M}^{*} \Omega\right)=0$, or

$$
i_{M}^{*} \omega_{K_{\alpha}}=0
$$

where $\omega_{K_{\alpha}}=g K_{\alpha}$ is the $(1,1)$-form associated with the almost complex structure

$$
K_{\alpha}=K \cos \alpha-J \sin \alpha .
$$

Therefore, $M$ is a Lagrangian submanifold with respect to both $\omega_{I}$ and $\omega_{K_{\alpha}}$. Since $I$ and $K_{\alpha}$ swap $T M$ and $N M$, the action of the almost complex structure

$$
J_{\alpha}=K_{\alpha} I=J \cos \alpha+K \sin \alpha
$$

leaves $T M$ invariant. In other words, $M$ is holomorphic in $J_{\alpha}$. It follows that $\omega_{J_{\alpha}}=g J_{\alpha}$ is nondegenerate on $T M$, so $M$ is a symplectic manifold with symplectic form $i_{M}^{*} \omega_{J_{\alpha}}$.

The quantization condition (2.95) translates to the condition

$$
\left[i_{M}^{*} \Omega\right] \in 2 \epsilon H^{2}(M ; \mathbb{Z}),
$$

which, in view of the condition (3.30), is equivalent to

$$
\left[i_{M}^{*} \omega_{J_{\alpha}}\right] \in 2 \pi \hbar H^{2}(M ; \mathbb{Z}) .
$$

This condition means that $A / \hbar$, with

$$
A=\operatorname{Re}\left(e^{-\mathrm{i} \alpha} i_{M}^{*} \Lambda\right),
$$

is a connection on a complex line bundle over $M$ with curvature

$$
\frac{1}{\hbar} \mathrm{d} A=\frac{1}{\hbar} i_{M}^{*} \omega_{J_{\alpha}}
$$

The localization formula (2.93), applied to the present setup, gives

$$
\int_{L} \operatorname{vol}_{L} \exp \left(\frac{\mathrm{i}}{\hbar} \int_{\mathbb{M}_{1}} \varphi^{*}\left(A-A_{0}\right)\right)
$$

where $A_{0}=\operatorname{Re}\left(e^{-\mathrm{i} \alpha} \Lambda_{0}\right)$ is a flat connection. In terms of real Darboux coordinates $\left(p_{\beta}, q^{\beta}\right)$ on $M$ such that

$$
A-A_{0}=p_{\beta} \mathrm{d} q^{\beta},
$$

we may write the above integral as

$$
\int_{L} \mathcal{D} p_{\beta} \mathcal{D} q^{\beta} \exp \left(\frac{\mathrm{i}}{\hbar} \int_{\mathbb{M}_{1}} p_{\beta} \partial_{t} q^{\beta} \mathrm{d} t\right)
$$

This is the path integral for quantum mechanics on $\mathbb{M}_{1}$ with vanishing Hamiltonian, quantizing the symplectic manifold $\left(M, i_{M}^{*} \omega_{J_{\alpha}}\right)$. 


\subsection{Reduction to the A-model}

In the above argument, we have established the equivalence between the $\Omega$-deformed Rozansky-Witten theory and quantum mechanics by directly writing down the localized expression for the path integral. We could have also done this in two steps, as we did in section 2, via the intermediate supersymmetric quantum mechanics which we obtain by cigar reduction. Doing so connects the $\Omega$-deformed Rozansky-Witten theory with the A-model setup studied by Gukov and Witten [1].

Let us recall the construction of the A-model [31]. The target space is a symplectic manifold $Y$, endowed with an almost complex structure and a compatible symplectic form. We will see that in the present context the almost complex structure is $K_{\alpha}$ and the symplectic form is $\omega_{K_{\alpha}}$, so we will use this notation. The A-model further depends on a choice of a closed real two-form $B$ on $Y$.

The worldsheet of the A-model is a Riemann surface $C$, whose complex structure we call $j$, equipped with a metric. On one-forms, $j$ acts from the right: if $z$ is a holomorphic coordinate on $C$, we have $\mathrm{d} z j=\mathrm{i} \mathrm{d} z$ and $\mathrm{d} \bar{z} j=-\mathrm{i} \mathrm{d} \bar{z}$.

The fields of the A-model are

$$
\begin{aligned}
& \phi \in \operatorname{Map}(C, Y), \\
& \psi \in \Pi \Omega^{0}\left(C, \phi^{*} T Y\right), \\
& \chi \in \Pi \Omega^{1}\left(C, \phi^{*} T Y\right), \\
& \mathrm{H} \in \Omega^{1}\left(C, \phi^{*} T Y\right) .
\end{aligned}
$$

The one-form fermion $\chi$ and auxiliary field $\mathrm{H}$ obey the self-duality constraint

$$
\begin{aligned}
\chi & =K_{\alpha} \chi j, \\
\mathrm{H} & =K_{\alpha} \mathrm{H} j .
\end{aligned}
$$

The A-model has a supercharge $Q$ which squares to zero. The covariant supersymmetry variations of the fields are

$$
\begin{aligned}
\delta \phi^{\mu} & =\psi^{\mu}, \\
\delta_{\nabla^{\prime}} \psi^{\mu} & =0 \\
\delta_{\nabla^{\prime}} \chi^{\mu} & =\mathrm{d} \phi^{\mu}+\left(K_{\alpha}\right)^{\mu}{ }_{\nu} \mathrm{d} \phi^{\nu} j+\mathrm{iH}^{\mu}, \\
\delta_{\nabla^{\prime}} \mathrm{H}^{\mu} & =\mathrm{i}\left(\mathrm{d}_{\nabla^{\prime}} \psi^{\mu}+\left(K_{\alpha}\right)^{\mu}{ }_{\nu} \mathrm{d}_{\nabla^{\prime}} \psi^{\nu} j\right)-\frac{\mathrm{i}}{2}\left(R_{\nabla^{\prime}}\right)^{\mu}{ }_{\nu \rho \sigma} \chi^{\nu} \psi^{\rho} \psi^{\sigma}+\psi^{\rho}\left(\nabla_{\rho}^{\prime} K_{\alpha}\right)^{\mu}{ }_{\nu} \mathrm{d} \phi^{\nu} j .
\end{aligned}
$$

As in supersymmetric quantum mechanics, $\nabla^{\prime}$ is a torsion-free connection on $Y$.

The action of the A-model is given by

$$
S_{\mathrm{A}}=\frac{1}{\hbar} \delta \int_{C} \frac{1}{4}\left(g_{\mu \nu} \chi^{\mu} \wedge \star\left(\mathrm{d} \phi^{\nu}+\left(K_{\alpha}\right)_{\rho}^{\nu} \mathrm{d} \phi^{\rho} j-\mathrm{i} \mathrm{H}^{\nu}\right)\right)+\frac{1}{\hbar} \int_{C} \phi^{*}\left(-\omega_{K_{\alpha}}+\mathrm{i} B\right) .
$$

The second integral is a topological term and therefore $Q$-invariant.

The equation of motion for the auxiliary field $\mathrm{H}$ is

$$
\mathrm{H}^{\mu}=\frac{\mathrm{i}}{2} \delta \phi^{\nu} g^{\mu \sigma} \nabla_{\nu}^{\prime} g_{\sigma \rho} \chi^{\rho},
$$


and the on-shell supersymmetry variation for $\chi$ is

$$
\delta_{\widetilde{\nabla}^{\prime}} \chi=\mathrm{d} \phi+K_{\alpha} \mathrm{d} \phi j,
$$

where $\widetilde{\nabla}^{\prime}$ is the metric connection (2.52). If $\mathrm{H}$ is integrated out, the bosonic part of the A-model action becomes

$$
\frac{1}{\hbar} \int_{C} \frac{1}{2} g_{\mu \nu} \mathrm{d} \phi^{\mu} \wedge \star \mathrm{d} \phi^{\nu}+\frac{\mathrm{i}}{\hbar} \int_{C} \phi^{*} B
$$

Let us return to the $\Omega$-deformed Rozansky-Witten theory. We place the theory on $\mathbb{R} \times \mathbb{S}^{1} \times \mathbb{M}_{1}$, and view it as the $\Omega$-deformed B-model on $\Sigma=\mathbb{R} \times \mathbb{S}^{1}$ with target $X=$ $\operatorname{Map}\left(\mathbb{M}_{1}, Y\right)$. Then, we reduce the theory on $\mathbb{S}^{1}$ and compare the resulting supersymmetric quantum mechanics with the A-model on $C=\mathbb{R} \times \mathbb{M}_{1}$ with target $Y$.

The coordinates on $\mathbb{R}$ and $\mathbb{M}_{1}$ are $s$ and $t$, respectively. For notational simplicity, we take the metric on $\mathbb{R}$ to be $\mathrm{d} s^{2}$ so that $\hat{s}$ can be replaced everywhere by $s$. We give $C$ a complex structure by declaring that

$$
z=s+\mathrm{i} t
$$

is a holomorphic coordinate. Then, we have

$$
j_{s}^{t}=-j^{s}{ }_{t}=1
$$

and $C$ is locally isomorphic to the complex plane $\mathbb{C}$ with the standard metric $\mathrm{d} s^{2}+\mathrm{d} t^{2}$.

For the superpotential (3.19), we have

$$
\frac{\delta h}{\delta \phi^{\mu}}=-\left(\omega_{K_{\alpha}}\right)_{\mu \nu} \partial_{t} \phi^{\nu},
$$

so the on-shell supersymmetry variation (2.50) of $\chi$ in supersymmetric quantum mechanics is

$$
\delta_{\widetilde{\nabla}^{\prime}} \chi_{s}^{\mu}=\partial_{s} \phi^{\mu}+\left(K_{\alpha}\right)^{\mu}{ }_{\nu} \partial_{t} \phi^{\nu} .
$$

This coincides with the supersymmetry variation of $\chi_{s}$ in the A-model.

In fact, all the supersymmetry variations of the fields match between the circle reduction of the $\Omega$-deformed Rozansky-Witten theory and the A-model. (The component $\chi_{t}$ of $\chi$ is determined from $\chi_{s}$ by the self-duality constraint.) The actions of the two theories also match, with the B-field given by

$$
B=\omega_{J_{\alpha}} .
$$

Thus, we have found that upon reduction on a circle, the $\Omega$-deformed Rozansky-Witten theory with complex symplectic target $Y$ and complex structure $I$ becomes the A-model with target $Y$, symplectic structure $\omega_{K_{\alpha}}$ and B-field $\omega_{J_{\alpha}}$.

Next, let us consider the $\Omega$-deformed Rozansky-Witten theory on $\mathbb{D} \times \mathbb{M}_{1}$, and reduce it on the circle fibers of the cigar $\mathbb{D}$ to obtain the A-model on $\mathbb{I} \times \mathbb{M}_{1}$. We wish to identify the boundary conditions at $s=0$ and $\ell$. 
We have already seen that the brane at $s=\ell$ must be supported on a Lagrangian submanifold $M \subset Y$ with respect to $\omega_{K_{\alpha}}$. The boundary conditions (2.82)-(2.85) in supersymmetric quantum mechanics translates to the boundary conditions

$$
\begin{aligned}
\phi & \in M, \\
\psi & \in T_{\phi} M \otimes \mathbb{C}, \\
\chi_{s} & \in N_{\phi} M \otimes \mathbb{C}, \\
\partial_{s} \phi & \in N_{\phi} M
\end{aligned}
$$

in the A-model. Here we have used the fact that $\partial_{t} \phi$ is tangent to $M$, so $K_{\alpha}=g^{-1} \omega_{K_{\alpha}}$ sends it to a vector normal to $M$.

The boundary condition for $\phi$ is the Dirichlet condition. To rewrite the boundary conditions for the fermions in a more familiar form, one may switch to the notation $\psi_{ \pm}$, commonly used in $\mathcal{N}=(1,1)$ supersymmetric sigma model. These fermions are related to $\psi, \chi_{s}$ by

$$
\begin{aligned}
\psi & =\frac{1}{2}\left(1+\mathrm{i} K_{\alpha}\right) \psi_{+}+\frac{1}{2}\left(1-\mathrm{i} K_{\alpha}\right) \psi_{-}=\frac{1}{2}\left(\psi_{+}+\psi_{-}\right)+\frac{\mathrm{i}}{2} K_{\alpha}\left(\psi_{+}-\psi_{-}\right), \\
\chi_{s} & =-\frac{\mathrm{i}}{4}\left(1-\mathrm{i} K_{\alpha}\right) \psi_{+}+\frac{\mathrm{i}}{4}\left(1+\mathrm{i} K_{\alpha}\right) \psi_{-}=-\frac{1}{4} K_{\alpha}\left(\psi_{+}+\psi_{-}\right)-\frac{\mathrm{i}}{4}\left(\psi_{+}-\psi_{-}\right) .
\end{aligned}
$$

In terms of $\psi_{ \pm}$, the boundary conditions for the fermions is

$$
\begin{aligned}
& \psi_{+}+\psi_{-} \in T_{\phi} M, \\
& \psi_{+}-\psi_{-} \in N_{\phi} M .
\end{aligned}
$$

These are the boundary conditions for a Lagrangian A-brane, that is, a boundary condition in $\mathcal{N}=(2,2)$ supersymmetric sigma model that preserves half of the four supercharges, including the one used in the construction of the A-model.

Unlike the Lagrangian brane at $s=\ell$, the boundary conditions at $s=0$ does not restrict the value of $\phi$. There is a natural candidate for the corresponding brane: a canonical coisotropic brane [32], whose support is the entire target space.

Indeed, the boundary conditions at $s=0$ require $(1-\mathrm{i} I) \psi=(1-\mathrm{i} I) \chi=0$, which is equivalent to demanding

$$
\psi_{+}=-J_{\alpha} \psi_{-} .
$$

This is precisely the boundary condition on the fermions imposed by the canonical coisotropic brane whose Chan-Paton line bundle carries a connection with curvature $\omega_{J_{\alpha}}$.

On the bosonic field, the boundary condition simply requires $\phi$ to obey the equation for a $K_{\alpha}$-holomorphic curve

$$
\partial_{s} \phi+K_{\alpha} \partial_{t} \phi=0 .
$$

If $K_{\alpha}$ is integrable, this equation says that $\phi$ is a holomorphic map. This is the familiar localization property of the A-model.

Thus, we have essentially shown that the boundary condition at $s=0$ is the canonical coisotropic brane, with the curvature of the Chan-Paton connection being $\omega_{J_{\alpha}}$. For this 
identification to make sense, however, $\omega_{J_{\alpha}}$ must satisfy the quantization condition on $Y$, not just on $M$ :

$$
\left[\omega_{J_{\alpha}}\right] \in 2 \pi \hbar H^{2}(Y ; \mathbb{Z}) .
$$

This is simply because the canonical coisotropic brane is supported everywhere on $Y$, and the curvature of a connection on any complex line bundle obeys the quantization condition.

Let us assume that this quantization condition is satisfied, and verify that the ChanPaton connection in question is present in the action. Under this assumption, the action is given, up to $Q$-exact terms, by the formula (2.98):

$$
-\frac{1}{\hbar} \int_{\mathbb{I} \times \mathbb{M}_{1}} \omega_{K_{\alpha}}+\frac{\mathrm{i}}{\hbar} \int_{\{\ell\} \times \mathbb{M}_{1}} A_{0}-\frac{\mathrm{i}}{\hbar} \int_{\{0\} \times \mathbb{M}_{1}} A .
$$

The gauge field $A$ (extended to a one-form on all of $Y$ ) has curvature $\omega_{J_{\alpha}}$ and is indeed the Chan-Paton connection for the canonical coisotropic brane. The flat gauge field $A_{0}$ is the Chan-Paton connection for the Lagrangian A-brane.

To summarize, the $\Omega$-deformed Rozansky-Witten theory on $\mathbb{D} \times \mathbb{M}_{1}$, reduced on the circle fibers of $\mathbb{D}$, is equivalent to the A-model on $\mathbb{I} \times \mathbb{M}_{1}$, with the canonical coisotropic brane with the Chan-Paton curvature $\omega_{J_{\alpha}}$ at $s=0$ and a Lagrangian brane at $s=\ell$ whose support $M$ is Lagrangian with respect to $\omega_{I}$ and $\omega_{K_{\alpha}}$ and holomorphic in $J_{\alpha}$. This A-model setup quantizes $M$, viewed as a symplectic manifold with symplectic form $i_{M}^{*} \omega_{J_{\alpha}}$.

\subsection{Hamiltonians}

As we have seen, quantum mechanics arising from the $\Omega$-deformed Rozansky-Witten theory on $\mathbb{D} \times \mathbb{M}_{1}$ has vanishing Hamiltonian. The reason can be traced back to the topological invariance of Rozansky-Witten theory, which in particular implies that translation along the time axis $\mathbb{M}_{1}$ is trivial.

To get quantum mechanics with a nontrivial Hamiltonian, one must modify the theory and break topological invariance on $\mathbb{M}_{1}$, while preserving the supercharge $Q_{V}$. Once Rozansky-Witten theory is expressed as a B-model, one can readily achieve such a modification with introduction of a nontopological term to the superpotential.

Let $w$ be an $I$-holomorphic function on $Y$, and suppose that the Lagrangian brane $M$ is chosen in such a way that $\operatorname{Im}(w / \epsilon)=0$ on $M$. (More generally, $w$ may have an explicit dependence on $t$.) We add the following term to the superpotential (3.19):

$$
\Delta W(\varphi)=\frac{\mathrm{i}}{2} \int_{\mathbb{M}_{1}} \varphi^{*} w \mathrm{~d} t .
$$

Then, the localized path integral becomes

$$
\int_{L} \operatorname{vol}_{L} \exp \left(\frac{\mathrm{i}}{\hbar} \int_{\mathbb{M}_{1}} \varphi^{*}\left(A-A_{0}-H \mathrm{~d} t\right)\right)
$$

where

$$
H=-\operatorname{Re}\left(e^{-\mathrm{i} \alpha} w\right) .
$$

Therefore, the $\Omega$-deformed Rozansky-Witten theory, modified by this additional superpotential, is equivalent to quantum mechanics with Hamiltonian $H$. 
Conversely, if a real function $H$ on $M$ can be extended to an $I$-holomorphic function on $Y$, we can devise a modification of Rozansky-Witten theory so that upon the $\Omega$-deformation it reduces to quantum mechanics on $M$ with Hamiltonian $H$.

Although this is a perfectly good way to introduce a nonzero Hamiltonian from the point of view of Rozansky-Witten theory, it makes the relation to the A-model less straightforward. As a matter of fact, for the Hamiltonian to be realized in the A-model, $H$ must be rather special.

In the A-model, the Hamiltonian originates, if at all, from a superpotential. A superpotential in the A-model is a one-form with values in real functions on $Y$ :

$$
Z \in \Omega^{1}\left(C, \phi^{*} \mathcal{O}_{Y}\right)
$$

It obeys the constraint

$$
\partial_{\mu} Z+\partial_{\nu} Z\left(K_{\alpha}\right)^{\nu}{ }_{\mu} j=0 .
$$

If $K_{\alpha}$ is integrable, this constraint means that we can write

$$
Z=w^{\prime} \mathrm{d} z+\bar{w}^{\prime} \mathrm{d} \bar{z}=2 \operatorname{Re}\left(w^{\prime}\right) \mathrm{d} s-2 \operatorname{Im}\left(w^{\prime}\right) \mathrm{d} t
$$

for some $K_{\alpha}$-holomorphic function $w^{\prime}$ on $Y$.

The superpotential enters the A-model through the action

$$
S_{\mathrm{A}, Z}=-\frac{1}{\hbar} \delta \int_{C} \frac{1}{2} \chi^{\mu} \wedge \partial_{\mu} Z+\frac{1}{\hbar} \int_{C} \mathrm{~d} Z .
$$

The addition of this action shifts the equation of motion for $\mathrm{H}$ by $\Delta \mathrm{H}^{\mu}=-\mathrm{i} g^{\mu \nu} \star \partial_{\nu} Z$, and produces the potential

$$
\frac{1}{\hbar} \int_{C} \frac{1}{4} g^{\mu \nu} \partial_{\mu} Z \wedge \star \partial_{\nu} Z
$$

For $C=\mathbb{I} \times \mathbb{M}_{1}$, the supersymmetry transformations of the A-model with superpotential $Z$ coincide with those of supersymmetric quantum mechanics on $\mathbb{I}$, obtained by cigar reduction of the $\Omega$-deformed Rozansky-Witten theory with the modified superpotential, if we identify

$$
Z_{t}=-\operatorname{Im}\left(e^{-\mathrm{i} \alpha} w\right) .
$$

The actions also match, provided that the boundary couplings

$$
-\frac{\mathrm{i}}{\hbar} \int_{\partial C} \mathrm{~d} H-\frac{\mathrm{i}}{\hbar} \int_{\{\ell\} \times \mathbb{M}_{1}} e^{-\mathrm{i} \alpha} w \mathrm{~d} t=-\frac{\mathrm{i}}{\hbar} \int_{\{0\} \times \mathbb{M}_{1}} H \mathrm{~d} t-\frac{1}{\hbar} \int_{\{\ell\} \times \mathbb{M}_{1}} Z_{t} \mathrm{~d} t
$$

are included in the A-model action. The first term on the left-hand side accounts for the change in the B-field in the A-model, while the second term accounts for the change in the boundary coupling in the $\Omega$-deformed Rozansky-Witten theory. These boundary terms combine with the second term in $S_{A, Z}$ to form the $I$-holomorphic boundary coupling

$$
-\frac{\mathrm{i}}{\hbar} \int_{\{0\} \times \mathbb{M}_{1}} e^{-\mathrm{i} \alpha} w \mathrm{~d} t
$$

which is $Q$-invariant by the boundary condition $(1-\mathrm{i} I) \psi=0$ at $s=0$. 
We have found that for this A-model construction to work, the Hamiltonian must be the real part of the $I$-holomorphic function $-e^{-\mathrm{i} \alpha} w$ whose imaginary part is the imaginary part of a $K_{\alpha}$-holomorphic function $-2 w^{\prime}$.

This is a strong constraint, but as pointed out in [9], there is a natural way to construct a Hamiltonian obeying this condition if $Y$ is a hyperkähler manifold that admits a Hamiltonian action of a Lie group preserving the hyperkähler structure. Using the moment maps $\mu_{I}, \mu_{J_{\alpha}}, \mu_{K_{\alpha}}$ for this Hamiltonian hyperkähler action with respect to the symplectic structures $\omega_{I}, \omega_{J_{\alpha}}, \omega_{K_{\alpha}}$, one defines $w=\mathrm{i}\left(\mu_{J}+\mathrm{i} \mu_{K}\right)$ and $w^{\prime}=-\left(\mu_{I}+\mathrm{i} \mu_{J_{\alpha}}\right) / 2$. Then, $H=\mu_{K_{\alpha}}$ and this Hamiltonian satisfies the required condition.

\section{Gauge symmetry}

So far we have studied the relation between A-type and B-type constructions in the case in which the theories involved do not possess gauge symmetry. In this section, we extend the analysis in section 2 to incorporate gauge symmetry. We will formulate the $\Omega$-deformation of the gauged B-model, and establish the equivalences between the $\Omega$-deformed gauged B-model on a disk, supersymmetric gauged quantum mechanics on an interval, and a zerodimensional gauged sigma model with complexified gauge group.

\section{$4.1 \Omega$-deformed gauged B-model}

The $\Omega$-deformation of B-twisted gauge theories with linear targets was formulated in [18]. Here we generalize this formulation so that the target space is allowed to be curved. We will focus on the Kähler case to avoid being overly technical, though the discussion that follows can be modified to accommodate non-Kähler target spaces. In any event, we will not encounter such target spaces in the gauge theory applications we will study.

Let $G$ be a compact Lie group (or the space of maps from $\mathbb{M}_{d}$ to a compact Lie group $H$ in higher-dimensional applications), and $G_{\mathbb{C}}$ its complexification. We choose a basis $\left\{T_{a}\right\}$ of the Lie algebra $\mathfrak{g}$ of $G$. Our convention is such that $T_{a}$ are represented by antihermitian matrices in a unitary representation of $G$.

The target space of the gauged B-model [42] is a Kähler manifold $X$ that admits a

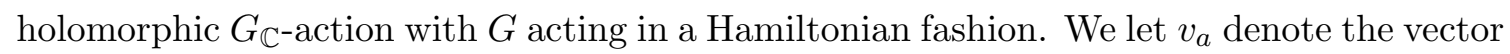

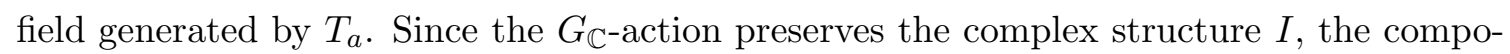
nents $v_{a}^{i}$ of $v_{a}$ are holomorphic functions, while $v_{a}^{\bar{i}}=\overline{v_{a}^{i}}$ are antiholomorphic functions. If $\left\{T_{a}\right\}$ satisfy the commutation relations $\left[T_{a}, T_{b}\right]=f_{a b} T_{c}$, then $\left[v_{a}, v_{b}\right]=-f_{a b} v_{c}$.

The $G$-action being Hamiltonian means that there are real functions $\left\{\mu_{a}\right\}$ such that $\mathrm{d} \mu_{a}=\iota_{v_{a}} \omega$, where $\omega$ is the Kähler form. As a consequence, the $G$-action preserves the Kähler form: $\mathcal{L}_{v_{a}} \omega=\left(\mathrm{d} \iota_{v_{a}}+\iota_{v_{a}} \mathrm{~d}\right) \omega=\mathrm{d}^{2} \mu_{a}=0$. Since $\mathcal{L}_{v_{a}} I=0$ and the Kähler metric $g$ is given by $g=-\omega I$, the $G$-action is an isometry. The functions $\left\{\mu_{a}\right\}$ define the moment map $\mu: X \rightarrow \mathfrak{g}^{*}$ by $\mu_{a}=\left\langle\mu, T_{a}\right\rangle$. We assume that $\mu$ is $G$-equivariant, that is, $\langle\mu(g \cdot x), \xi\rangle=$ $\left\langle\mu(x), \operatorname{Ad}_{g^{-1}} \xi\right\rangle$ for any $g \in G, \xi \in \mathfrak{g}$.

In addition to the chiral multiplet fields (2.1)-(2.6), the gauged B-model has a gauge field which is locally a one-form valued in $\mathfrak{g}$,

$$
A \in \Omega^{1}(\Sigma, \mathfrak{g}),
$$


as well as bosonic fields

$$
\begin{aligned}
& \sigma \in \Omega^{1}(\Sigma, \mathfrak{g}), \\
& \mathrm{D} \in \Omega^{0}(\Sigma, \mathfrak{g})
\end{aligned}
$$

and fermionic fields

$$
\begin{aligned}
& \alpha \in \Pi \Omega^{0}(\Sigma, \mathfrak{g}), \\
& \lambda \in \Pi \Omega^{1}(\Sigma, \mathfrak{g}), \\
& \zeta \in \Pi \Omega^{2}(\Sigma, \mathfrak{g}),
\end{aligned}
$$

all in the adjoint representation. These fields form a vector multiplet.

It is convenient to define complex gauge fields

$$
\begin{aligned}
& \mathcal{A}=A+\mathrm{i} \sigma, \\
& \overline{\mathcal{A}}=A-\mathrm{i} \sigma .
\end{aligned}
$$

The field strengths of $A$ is $F=\mathrm{d} A+A \wedge A$. In a like manner, we define $\mathcal{F}=\mathrm{d} \mathcal{A}+\mathcal{A} \wedge \mathcal{A}$ and $\overline{\mathcal{F}}=\mathrm{d} \overline{\mathcal{A}}+\overline{\mathcal{A}} \wedge \overline{\mathcal{A}}$.

The $\Omega$-deformed supercharge $Q_{V}$ acts on the vector multiplet by

$$
\begin{aligned}
\delta \mathcal{A} & =\iota_{V} \zeta, \\
\delta \overline{\mathcal{A}} & =2 \lambda-\iota_{V} \zeta, \\
\delta \lambda & =\iota_{V} F-\mathrm{id}_{A} \iota_{V} \sigma, \\
\delta \zeta & =\mathcal{F}, \\
\delta \alpha & =\mathrm{D}, \\
\delta \mathrm{D} & =\iota_{V} \mathrm{~d}_{\mathcal{A}} \alpha .
\end{aligned}
$$

The exterior derivatives coupled to the gauge fields act on the vector multiplet in the usual manner. For instance, $\mathrm{d}_{\mathcal{A}} \alpha=\mathrm{d} \alpha+[\mathcal{A}, \alpha]$.

On the chiral multiplet, $Q_{V}$ acts by

$$
\begin{aligned}
\delta \varphi^{i} & =\iota_{V} \rho^{i}, \\
\delta \rho^{i} & =\mathrm{d}_{\mathcal{A}} \varphi^{i}+\iota_{V} \mathrm{G}^{i}, \\
\delta \mathrm{G}^{i} & =\mathrm{d}_{\mathcal{A}} \rho^{i}-\zeta^{a} v_{a}^{i}, \\
\delta \bar{\varphi}^{\bar{\imath}} & =\eta^{\bar{\imath}} \\
\delta \eta^{\bar{\imath}} & =\iota_{V} \mathrm{~d}_{\mathcal{A}} \bar{\varphi}^{\bar{\imath}}, \\
\delta \mu^{\bar{\imath}} & =\overline{\mathrm{G}}^{\bar{\imath}}, \\
\delta \overline{\mathrm{G}}^{\bar{\imath}} & =\mathrm{d}_{\mathcal{A}} \iota_{V} \mu^{\bar{\imath}} .
\end{aligned}
$$

The action of $\mathrm{d}_{\mathcal{A}}$ on the chiral multiplet fields is determined by $v_{a}$. We have

$$
\begin{aligned}
& \mathrm{d}_{\mathcal{A}} \varphi^{i}=\mathrm{d} \varphi^{i}+\mathcal{A}^{a} v_{a}^{i}, \\
& \mathrm{~d}_{\mathcal{A}} \rho^{i}=\mathrm{d} \rho^{i}+\mathcal{A}^{a} \partial_{j} v_{a}^{i} \wedge \rho^{j},
\end{aligned}
$$


and similarly,

$$
\begin{aligned}
& \mathrm{d}_{\mathcal{A}} \bar{\varphi}^{\bar{\imath}}=\mathrm{d} \bar{\varphi}^{\bar{\imath}}+\mathcal{A}^{a} v_{a}^{\bar{\imath}}, \\
& \mathrm{d}_{\mathcal{A}} \mu^{\bar{\imath}}=\mathrm{d} \mu^{\bar{\imath}}+\mathcal{A}^{a} \partial_{\bar{\jmath}} v_{a}^{\bar{\imath}} \wedge \mu^{\bar{\jmath}} .
\end{aligned}
$$

In the $\Omega$-deformed gauged B-model, the $G$-action is gauged. If $\varepsilon$ is a $\mathfrak{g}$-valued function on $\Sigma$, the infinitesimal gauge transformation $\delta_{\varepsilon}$ with parameter $\varepsilon$ acts by

$$
\begin{aligned}
\delta_{\varepsilon} \varphi^{i} & =\varepsilon^{a} v_{a}^{i}, \\
\delta_{\varepsilon} \rho^{i} & =\varepsilon^{a} \partial_{j} v_{a}^{i} \rho^{j}
\end{aligned}
$$

and so on. The operator $\mathrm{d}_{\mathcal{A}}$ does not commute with gauge transformations: although $\mathrm{d}_{\mathcal{A}} \varphi^{i}$ transforms covariantly,

$$
\delta_{\varepsilon}\left(\mathrm{d}_{\mathcal{A}} \varphi^{i}\right)=\varepsilon^{a} \partial_{j} v_{a}^{i} \mathrm{~d}_{\mathcal{A}} \varphi^{j},
$$

the gauge transformation of $\mathrm{d}_{\mathcal{A}} \rho^{i}$ contains a nonhomogeneous term:

$$
\delta_{\varepsilon}\left(\mathrm{d}_{\mathcal{A}} \rho^{i}\right)=\varepsilon^{a} \partial_{j} v_{a}^{i} \mathrm{~d}_{\mathcal{A}} \rho^{i}+\varepsilon^{a} \partial_{k} \partial_{j} v_{a}^{i} \mathrm{~d}_{\mathcal{A}} \varphi^{k} \wedge \rho^{j} .
$$

To construct a gauge covariant derivative, we need to pick a connection $\nabla$. Recall that $\nabla$ should be torsion-free and preserve the complex structure. Moreover, we require it to be $G$-invariant:

$$
\left(\mathcal{L}_{v_{a}} \Gamma\right)_{\nu \rho}^{\mu}=\nabla_{\nu} \nabla_{\rho} v_{a}^{\mu}-\left(R_{\nabla}\right)^{\mu}{ }_{\rho \nu \sigma} v_{a}^{\sigma}=0 .
$$

This equation can be rewritten as

$$
v_{a}\left(\Gamma_{\nu \rho}^{\mu}\right)=\partial_{\sigma} v_{a}^{\mu} \Gamma_{\nu \rho}^{\sigma}-\partial_{\nu} v_{a}^{\sigma} \Gamma_{\sigma \rho}^{\mu}-\partial_{\rho} v_{a}^{\sigma} \Gamma_{\nu \sigma}^{\mu}-\partial_{\nu} \partial_{\rho} v_{a}^{\mu}
$$

The left-hand side is the gauge transformation of $\Gamma$ with parameter $\varepsilon=T_{a}$, whereas the right-hand side is the action of the diffeomorphism on $\Gamma$ generated by $T_{a}$. In other words, this condition ensures that a gauge transformation acts on $\Gamma$ as the corresponding diffeomorphism.

Since the $G$-action is an isometry and $X$ is Kähler, one choice of $\nabla$ is the LeviCivita connection. The above condition can be readily checked: from the Killing equation $\nabla_{\mu}\left(v_{a}\right)_{\nu}+\nabla_{\nu}\left(v_{a}\right)_{\mu}=0$, one gets $\nabla_{k} v_{a}^{i}=-g^{i \bar{l}} g_{\bar{m} k} \nabla_{\bar{l}} v_{a}^{k}$ and

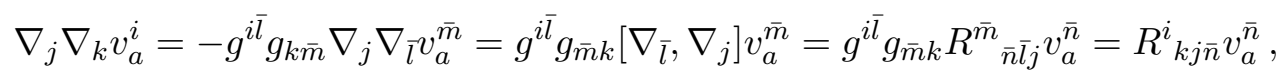

where the equations $\nabla_{j} v_{a}^{\bar{m}}=\partial_{j} v_{a}^{\bar{m}}=0$ is used in the second equality.

Using $\nabla$, we define the gauge covariant derivative $\mathrm{d}_{\mathcal{A}, \nabla}$ by coupling $\mathrm{d}_{\mathcal{A}}$ to the pullback of $\nabla$. For example,

$$
\mathrm{d}_{\mathcal{A}, \nabla} \rho^{i}=\mathrm{d}_{\mathcal{A}} \rho^{i}+\mathrm{d}_{\mathcal{A}} \varphi^{j} \Gamma_{j k}^{i} \wedge \rho^{k} .
$$

In the gauge transformation of $\mathrm{d}_{\mathcal{A}, \nabla}$, the nonhomogeneous terms coming from $\mathrm{d}_{\mathcal{A}}$ and $\mathrm{d}_{\mathcal{A}} \varphi^{j} \Gamma_{j k}^{i}$ cancel. Thus, $\mathrm{d}_{\mathcal{A}, \nabla}$ commutes with the gauge symmetry. 
The supersymmetry transformations satisfy

$$
\delta_{Q_{V}}^{2}=\mathrm{d}_{\mathcal{A}} \iota_{V}+\iota_{V} \mathrm{~d}_{\mathcal{A}},
$$

except when $\delta_{Q_{V}}^{2}$ acts on $G, \bar{G}$, which yields extra terms involving second derivatives of $v_{a}$. These terms are present because $\mathrm{G}, \overline{\mathrm{G}}$ are not tensors. Rather, the auxiliary fields $\mathrm{F}, \overline{\mathrm{F}}$ are tensors, and $G, \bar{G}$ differ from them by terms depending on $\Gamma$; see the definitions (2.23) and (2.24). The above relation holds on $F, \bar{F}$.

The diffeomorphism covariant version of the supersymmetry transformations is

$$
\begin{aligned}
& \delta \varphi^{i}=\iota_{V} \rho^{i}, \\
& \delta_{\nabla} \rho^{i}=\mathrm{d}_{\mathcal{A}} \varphi^{i}+\iota_{V} \mathrm{~F}^{i}, \\
& \delta_{\nabla} \mathrm{F}^{i}=\mathrm{d}_{\mathcal{A}, \nabla} \rho^{i}-\zeta^{a} v_{a}^{i}-\left(\frac{1}{3}\left(R_{\nabla}\right)^{i}{ }_{j k l} \iota_{V} \rho^{l}+\frac{1}{2}\left(R_{\nabla}\right)^{i}{ }_{j k l}{ }^{\bar{l}} \eta^{\bar{l}}\right) \rho^{j} \wedge \rho^{k}, \\
& \delta \bar{\varphi}^{\bar{\imath}}=\eta^{\bar{\imath}}, \\
& \delta_{\nabla} \eta^{\bar{\imath}}=\iota_{V} \mathrm{~d}_{\mathcal{A}} \bar{\varphi}^{\bar{\imath}} \\
& \delta_{\nabla} \mu^{\bar{\imath}}=\overline{\mathrm{F}}^{\bar{\imath}}
\end{aligned}
$$

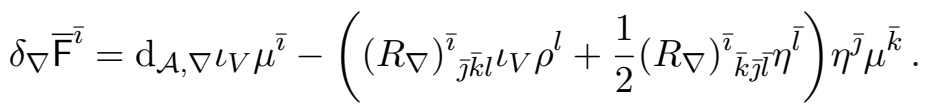

The worldsheet derivatives that appear on the right-hand sides are all gauge covariant, though $\delta_{\nabla}$ itself does not commute with gauge transformation.

The action of the $\Omega$-deformed gauged B-model consists of three pieces:

$$
S_{\Omega \mathrm{GB}}=S_{\Omega \mathrm{GB}, \mathrm{V}}+S_{\Omega \mathrm{GB}, \mathrm{C}}+S_{\Omega \mathrm{GB}, W} .
$$

The first piece is the action for the vector multiplet and given by

$$
S_{\Omega \mathrm{GB}, \mathrm{V}}=\delta_{Q_{V}} \int_{\Sigma} \operatorname{Tr}\left(\left(-\star \mathrm{D}+\frac{2 \mathrm{i}}{1+\|V\|^{2}}\left(\mathrm{~d}_{A} \star \sigma+\star \iota_{\bar{V}} \mathrm{~d}_{A} \iota_{V} \sigma\right)\right) \alpha-\frac{1}{1+\|V\|^{2}} \zeta \star \overline{\mathcal{F}}\right),
$$

where $\operatorname{Tr}$ is a negative semidefinite bilinear form on $\mathfrak{g}$. We have chosen this particular integrand so that after the auxiliary fields are integrated out, the bosonic part of the action takes a nice form, free of cross terms. The second one is the chiral multiplet action:

$$
S_{\Omega \mathrm{GB}, \mathrm{C}}=\delta_{Q_{V}} \int_{\Sigma}\left(g_{\bar{\imath} j}\left(\mathrm{~d}_{\overline{\mathcal{A}}} \bar{\varphi}^{\bar{\imath}}+\iota \bar{V}_{\overline{\mathrm{F}}}^{\bar{\imath}}\right) \wedge \star \rho^{j}+g_{\bar{\imath} j} \mu^{\bar{\imath}} \star \mathrm{F}^{j}+\star \mathrm{i} \mu_{a} \alpha^{a}\right) .
$$

The third is the superpotential action and takes the same form as in the nongauged case:

$$
S_{\Omega \mathrm{GB}, W}=\int_{\Sigma}\left(\mathrm{F}^{i} \partial_{i} W+\frac{1}{2} \rho^{i} \wedge \rho^{j} \nabla_{i} \partial_{j} W-\delta_{Q_{V}}\left(\mu^{\bar{\imath}} \partial_{\bar{\imath}} \bar{W}\right)\right)-\int_{\partial \Sigma} W \frac{\mathrm{d} \theta}{V^{\theta}} .
$$

The superpotential $W$ must be gauge invariant:

$$
v_{a}(W)=0 .
$$

This condition implies that $\partial_{i} W$ and $\nabla_{i} \partial_{j} W$ transform under the gauge symmetry as tensors, and the superpotential action is gauge invariant. 


\subsection{Reduction to supersymmetric gauged quantum mechanics}

Taking $\Sigma=\mathbb{R} \times \mathbb{S}^{1}$ and keeping only the zero modes along $\mathbb{S}^{1}$ in the $\Omega$-deformed gauged B-model, we get a topologically twisted supersymmetric gauged quantum mechanics on $\mathbb{R}$. We recall that the metric on $\mathbb{R} \times \mathbb{S}^{1}$ is $\mathrm{d} \hat{s}^{2}+|\epsilon|^{-2} \mathrm{~d} \theta^{2}$ and $V=\epsilon \partial_{\theta}=\partial_{\hat{\theta}}$.

Supersymmetric gauged quantum mechanics that we obtain is constructed from a vector multiplet consisting of fields

$$
\begin{aligned}
A & \in \Omega^{1}(\mathbb{R}, \mathfrak{g}), \\
\sigma & \in \Omega^{1}(\mathbb{R}, \mathfrak{g}), \\
\tau & \in \Omega^{0}(\mathbb{R}, \mathfrak{g} \otimes \mathbb{C}), \\
\mathrm{D} & \in \Omega^{0}(\mathbb{R}, \mathfrak{g}) \\
\alpha & \in \Pi \Omega^{0}(\mathbb{R}, \mathfrak{g}), \\
\lambda & \in \Pi \Omega^{1}(\mathbb{R}, \mathfrak{g}), \\
\kappa & \in \Pi \Omega^{0}(\mathbb{R}, \mathfrak{g}), \\
\xi & \in \Pi \Omega^{1}(\mathbb{R}, \mathfrak{g}),
\end{aligned}
$$

and a chiral multiplet with fields (2.39)-(2.42).

Supersymmetry transforms the vector multiplet fields as

$$
\begin{aligned}
\delta A & =\lambda, \\
\delta \sigma & =\xi, \\
\delta \tau & =0, \\
\delta \bar{\tau} & =\kappa, \\
\delta \lambda & =-\mathrm{d}_{A} \tau, \\
\delta \xi & =[\tau, \sigma], \\
\delta \kappa & =[\tau, \bar{\tau}], \\
\delta \alpha & =\mathrm{D}, \\
\delta \mathrm{D} & =[\tau, \alpha],
\end{aligned}
$$

and the chiral multiplet fields as

$$
\begin{aligned}
\delta \phi^{\mu}= & \psi^{\mu} \\
\delta_{\nabla^{\prime}} \psi^{\mu}= & \tau^{a} v_{a}^{\mu} \\
\delta_{\nabla^{\prime}} \chi^{\mu}= & \mathrm{d}_{A} \phi^{\mu}+\sigma^{a} I^{\mu}{ }_{\nu} v_{a}^{\nu}+\mathrm{iH}^{\mu} \\
\delta_{\nabla^{\prime}} \mathrm{H}^{\mu}= & \mathrm{id}{ }_{A, \nabla^{\prime}} \psi^{\mu}+\mathrm{i} \lambda^{a} v_{a}^{\mu}-\xi^{a} I^{\mu}{ }_{\nu} v_{a}^{\nu} \\
& \quad-\sigma^{a} I^{\mu}{ }_{\nu} \nabla_{\rho}^{\prime} v_{a}^{\nu} \psi^{\rho}-\mathrm{i} \tau^{a} \nabla_{\nu}^{\prime} v_{a}^{\mu} \chi^{\nu}-\frac{\mathrm{i}}{2}\left(R_{\nabla^{\prime}}\right)^{\mu}{ }_{\nu \rho \sigma} \chi^{\nu} \psi^{\rho} \psi^{\sigma} .
\end{aligned}
$$

We have the relation $\delta_{Q}^{2}=\delta_{\tau}$, where the right-hand side is the infinitesimal gauge transformation with parameter $\tau$. 
The action for supersymmetric gauged quantum mechanics is

$$
\begin{array}{r}
S_{\mathrm{SGQM}}=\frac{2}{\hbar} \delta_{Q} \int_{\mathbb{R}} \operatorname{Tr}\left(\star\left(-\mathrm{D}+\mathrm{i} \star \mathrm{d}_{A} \star \sigma-[\tau, \bar{\tau}]\right) \alpha+\frac{1}{2}(\lambda+\mathrm{i} \xi) \star \mathrm{d}_{\overline{\mathcal{A}}} \bar{\tau}\right) \\
+\frac{1}{\hbar} \delta_{Q} \int_{\mathbb{R}}\left(\frac{1}{2} g_{\mu \nu} \chi^{\mu} \star\left(\mathrm{d}_{A} \phi^{\nu}-\mathrm{i} \mathrm{H}^{\nu}\right)-\star \sigma^{a} \chi^{\mu} \partial_{\mu} \mu_{a}\right. \\
\left.+\star\left(g_{\mu \nu}+\mathrm{i} \omega_{\mu \nu}\right) \bar{\tau}^{a} v_{a}^{\mu} \psi^{\nu}+\star 2 \mathrm{i} \alpha^{a} \mu_{a}\right) \\
+\frac{1}{\hbar} \int_{\mathbb{R}}\left(-\delta_{Q}\left(\chi^{\mu} \partial_{\mu} h\right)+\mathrm{d} h+\mathrm{i} \phi^{*} a\right) .
\end{array}
$$

The potential $h$ is assumed to be invariant under the action of $G_{\mathbb{C}}$, not only $G$. This condition is satisfied if $h$ is the real part of a gauge invariant holomorphic function, which is the case when the theory is the reduction of the $\Omega$-deformed gauged B-model. Note that due to this assumption, supersymmetric gauged quantum mechanics knows about the complex structure of the Kähler target space $X$.

Integrating out the auxiliary fields, one finds that the bosonic part of the action is

$$
\begin{aligned}
\frac{1}{\hbar} \int_{\mathbb{R}}\left(\frac{1}{2}\left\|\mathrm{~d}_{A} \star \sigma\right\|^{2}\right. & +\left\|\mathrm{d}_{A} \tau\right\|^{2}+\frac{1}{2}\|[\tau, \bar{\tau}]\|^{2}+\|[\sigma, \tau]\|^{2} \\
& +\frac{1}{2}\left\|\mathrm{~d}_{A} \phi\right\|^{2}+\frac{1}{2}\|\mathrm{~d} h\|^{2}+\frac{1}{2}\|\mu\|^{2}+\left\|\sigma^{a} v_{a}\right\|^{2}+\left\|\tau^{a} v_{a}\right\|^{2} \\
& \left.-\star \mathrm{i}[\tau, \bar{\tau}]^{a} \mu_{a}-\star \mathrm{i} \tau^{a} \bar{\tau}^{b} v_{a}^{\mu} \partial_{\mu} \mu_{b}-\mathrm{d}\left(\mathrm{i} \operatorname{Tr}(\star \sigma[\tau, \bar{\tau}])+\star \sigma^{a} \mu_{a}\right)+2 \sigma^{a} I v_{a}(h)\right),
\end{aligned}
$$

where the norms of various tensors are defined with the metrics on $\mathbb{R}$ and $X$ as well as the positive bilinear form - Tr on $\mathfrak{g}$. The first two terms in the last line cancel by the equivariance of the moment map, which implies

$$
v_{a}\left(\mu_{b}\right)=-f_{a b}{ }^{c} \mu_{c}
$$

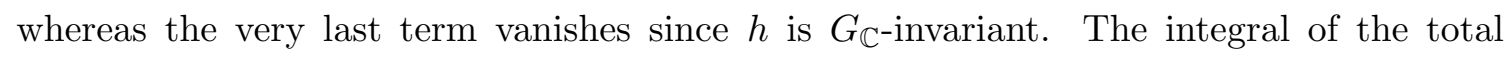
derivative terms vanishes by appropriate boundary conditions.

The on-shell supersymmetry transformations are given by

$$
\begin{aligned}
\delta \alpha & =\frac{\mathrm{i}}{2} \star \mathrm{d}_{A} \star \sigma-\frac{1}{2}[\tau, \bar{\tau}]-\frac{\mathrm{i}}{2} \mu^{\vee}, \\
\delta_{\widetilde{\nabla}} \chi & =\mathrm{d}_{A} \phi+\sigma^{a} I v_{a}-g^{-1} \mathrm{~d} h
\end{aligned}
$$

where $\mu^{\vee}$ is the dual of $\mu$ with respect to the metric $-\operatorname{Tr}$ on $\mathfrak{g}$.

As asserted already, upon reduction on $\mathbb{S}^{1}$, the $\Omega$-deformed gauged B-model becomes supersymmetric gauged quantum mechanics. The vector multiplet fields of the latter are 
expressed in terms of the zero modes of the vector multiplet fields of the former as follows:

$$
\begin{aligned}
\star A & =e^{-\mathrm{i} \alpha} \iota_{V} \star A_{0}, \\
\star \sigma & =e^{-\mathrm{i} \alpha} \iota_{V} \star \sigma_{0}, \\
\tau & =\iota_{V} \mathcal{A}_{0}, \\
\star \lambda & =e^{-\mathrm{i} \alpha} \iota_{V} \star \lambda_{0}, \\
\kappa & =2 \iota_{\bar{V}} \lambda_{0}, \\
\star \xi & =\mathrm{i} e^{-\mathrm{i} \alpha} \iota_{V} \star \lambda_{0}+\mathrm{i} e^{\mathrm{i} \alpha} \star \zeta_{0}, \\
\alpha & =\alpha_{0}, \\
\mathrm{D} & =\mathrm{D}_{0} .
\end{aligned}
$$

The left-hand sides refer to fields in supersymmetric quantum mechanics, the right-hand sides the $\Omega$-deformed gauged B-model. The chiral multiplet fields are identified as in the relations $(2.66)-(2.70)$ and

$$
\begin{aligned}
& \mathrm{H}^{i}=-\mathrm{i}\left(\mathrm{d}_{A_{0}} \varphi_{0}^{i}+\mathrm{i} \sigma_{0}^{a} v_{a}^{i}+2 \iota_{V} \mathrm{~F}_{0}^{i}\right), \\
& \mathrm{H}^{\bar{\imath}}=+\mathrm{i}\left(\mathrm{d}_{A_{0}} \bar{\varphi}_{0}^{\bar{\imath}}-\mathrm{i} \sigma_{0}^{a} v_{a}^{i}+2 \iota \bar{V}_{\overline{\mathrm{F}}}^{\overline{\overline{\mathrm{F}}}}\right) .
\end{aligned}
$$

Under the above identification of fields, the supersymmetry transformations of the $\Omega$ deformed gauged B-model reproduce those of supersymmetric gauged quantum mechanics. Likewise, the action for the zero modes of the $\Omega$-deformed B-model coincides with the action for supersymmetric gauged quantum mechanics.

To be precise, if supersymmetric gauged quantum mechanics is obtained by circle reduction, the real part of $\tau$ is periodic due to the gauge symmetry. A better characterization of $\tau$ is in terms of the holonomy of $\mathcal{A}$ around $\mathbb{S}^{1}$, which is valued in $G$ rather than $\mathfrak{g}$. That said, the topology of the target space does not really matter in the applications that we will consider, for in the end the path integral localizes to a locus where $\tau=0$.

\subsection{Boundary conditions}

Let us take $\Sigma=\mathbb{D}$ and discuss the boundary conditions for supersymmetric gauged quantum mechanics on $\mathbb{I}$, obtained by cigar reduction of the $\Omega$-deformed gauged B-model.

In the $\Omega$-deformed gauged B-model, we impose boundary conditions on $\partial \mathbb{D}$ such that the gauge symmetry is unbroken. Then, the gauge field should satisfy the Neumann condition $F=0$. We choose a gauge such that

$$
A_{s}=0
$$

on the boundary. Then, this condition reads

$$
\partial_{s} A_{\theta}=0 .
$$

We also require the boundary conditions to be $Q_{V}$-invariant. Taking the supersymmetry variations of the above conditions, we get

$$
\lambda_{s}=\partial_{s} \lambda_{\theta}=0,
$$


and taking further supersymmetry variations gives

$$
\partial_{s} \sigma_{\theta}=0
$$

Since the boundary values of $A_{\theta}$ and $\sigma_{\theta}$ are not fixed, to be compatible with the equation of motion for $\mathrm{D}$, the boundary value of $\mathrm{D}$ should not be fixed either. Since $\alpha$ and D are paired by supersymmetry, both $\alpha$ and D obey the Neumann condition:

$$
\partial_{s} \alpha=\partial_{s} \mathrm{D}=0
$$

Since half of the fermions obey the Neumann condition, the other half should obey the Dirichlet condition, and we get

$$
\zeta=0
$$

Supersymmetry then tells that $\sigma_{s}$ should also obey the Dirichlet condition:

$$
\sigma_{s}=0
$$

The boundary conditions for the chiral multiplet are not affected by the coupling to the vector multiplet, and specified by a submanifold $L \subset X$. The only additional condition compared to the nongauged case is that $L$ must be $G_{\mathbb{C}}$-invariant, which implies that $v_{a}$ is tangent to $L$ on the boundary.

Upon reduction to supersymmetric gauged quantum mechanics, the above boundary conditions for the vector multiplet become

$$
A=\sigma=\lambda=\xi=\mathrm{d} \tau=\mathrm{dD}=\mathrm{d} \kappa=\mathrm{d} \alpha=0 .
$$

In other words, all one-form fields and the derivatives of all zero-form fields vanish at $s=\ell$.

The boundary at $s=0$ comes from the tip of the cigar. Looking at the identifications (4.73)-(4.80), we deduce

$$
A=\sigma=\tau=\lambda=\kappa=0
$$

at $s=0$. The remaining fermions should obey the Neumann condition:

$$
\mathrm{d} \alpha=\mathrm{d} \star \xi=0 .
$$

The boundary conditions for the chiral multiplet is the same as in the nongauged case.

Note that the condition $A=0$ is really a gauge fixing condition, rather than a boundary condition, because a gauge transformation can shift the boundary value of $A$. However, the corresponding gauge transformation in the $\Omega$-deformed gauged B-model is singular at the tip of the cigar. Thus, the cigar geometry picks the gauge $A=0$ at $s=0$.

\subsection{Reduction to zero-dimensional gauged sigma model}

Shrinking the interval II to a point, we can reduce supersymmetric gauged quantum mechanics to a zero-dimensional gauge theory. Let us determine this theory. 
To simplify the analysis we choose the gauge $A=0$. We integrate the auxiliary fields out, rescale the metric of $\mathbb{I}$ by $u^{-2}$, and take the limit $u \rightarrow \infty$. Then, all bosonic fields get frozen to constants. By the boundary conditions at $s=0$, the bosonic fields in the vector multiplet are set to zero. By the boundary conditions at $s=\ell$, the bosonic field $\phi$ of the chiral multiplet must be valued in $L$.

When the gauge symmetry is absent, we have chosen the support $L$ of the brane at $s=\ell$ to be a Lagrangian submanifold of $X$. To understand what sort of submanifold $L$ should be in the presence of gauge symmetry, let us rescale the target metric $g$ by $u$ at the same time as the rescaling of the metric on $\mathbb{I}$. This entails rescaling of the moment map $\mu$ by $u$, and further constrains $\phi$ to be a zero of $\mu$ :

$$
\mu=0 \text {. }
$$

If we gauge the global gauge symmetry (which preserves the gauge $A=0$ and, due to the equivariance of $\mu$, the above equation), then the theory is effectively described by one without gauge symmetry, whose target space is

$$
X=\mu^{-1}(0) / G .
$$

The space $X$ is the symplectic quotient $X / / G$ of $X$, and is Kähler. Hence, we should take $L$ to be a submanifold whose image in $X$ is a Lagrangian submanifold $\mathcal{L}$.

The submanifold $L$ must also be $G_{\mathbb{C}}$-invariant, and this condition can be naturally satisfied. A key fact is that by a complex analytic version of the Kempf-Ness theorem, $X / / G$ is isomorphic to the quotient $X^{\mathrm{ss}} / G_{\mathbb{C}}$ of the set $X^{\mathrm{ss}}$ of semistable points of $X$ by $G_{\mathbb{C}}$. (A point in $X$ is said to be semistable if the closure of its $G_{\mathbb{C} \text {-orbit intersects }}$ $\mu^{-1}(0)$.) This fact shows that we may take $L$ to be the preimage of $\mathcal{L}$ under the projection $\pi: X^{\mathrm{ss}} \rightarrow X^{\mathrm{ss}} / G_{\mathbb{C}}:$

$$
L=\pi^{-1}(\mathcal{L})
$$

This is the support of a Lagrangian brane in the presence of gauged symmetry.

With this choice of $L$, the same argument as before immediately tells us that the fermion zero modes from the chiral multiplet that survive the boundary conditions are the constant modes of $\psi^{\bar{\imath}} \partial_{\bar{\imath}}$ and $\bar{\chi}^{\bar{\imath}} \partial_{\bar{\imath}}$ that descend to zero vectors in $X$. In other words, they must be in the kernel of $\pi_{*}$. The kernel is spanned by vector fields generated by $G_{\mathbb{C}}$ and, on $L$, sits inside $T L \otimes \mathbb{C}$. Since $\star \chi$ must be normal to $L$ at $s=\ell$, the zero mode of $\chi$ must vanish. However, the zero mode of $\psi$ may take the form

$$
\psi_{0}=\beta_{0}^{a} v_{a}^{\bar{\imath}} \partial_{\bar{\imath}}
$$

with $\beta_{0}$ being an arbitrary constant valued in $\Pi \mathfrak{g}$.

There is another fermion zero mode that pairs up with $\beta_{0}$. By looking at the boundary conditions, we see that the zero mode of $\alpha$ can be an arbitrary constant $\alpha_{0} \in \Pi \mathfrak{g}$. The only nonvanishing term in the action that contains these fermion zero modes is

$$
\frac{1}{\hbar} \int_{\mathbb{R}} 2 \mathrm{i} \star \beta^{b} v_{b}^{\bar{\imath}} \partial_{\bar{\imath}} \mu_{a} \alpha_{0}^{a}
$$


We can rewrite this term as

$$
\frac{1}{\hbar} \int_{\mathbb{R}}\left(-\star \beta_{0}^{b}\left(I v_{b}\right)\left(\mu_{a}\right) \alpha_{0}^{a}+\mathrm{i} \beta_{0}^{b} v_{b}\left(\mu_{a}\right) \alpha_{0}^{a}\right) .
$$

By the relation (4.70) the second term in the integrand vanishes on $\mu^{-1}(0)$, to which the path integral localizes. The first term has a nice interpretation.

The bosonic terms in the action for the zero-dimensional theory are those that appear

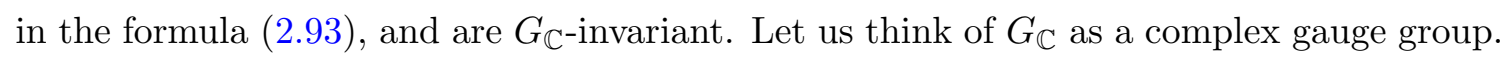
The isomorphism $\mu^{-1}(0) / G \cong X^{\text {ss }} / G_{\mathbb{C}}$ essentially says that the localization equation (4.93) is a partial gauge fixing condition: it breaks the gauge group $G_{\mathbb{C}}$ down to $G$. The first term in the above integral produces the Faddeev-Popov determinant associated with this partial gauge fixing, with $\alpha_{0}, \beta_{0}$ playing the roles of ghost fields.

Therefore, the $\Omega$-deformed gauged B-model on $\partial \mathbb{D}$, and the corresponding supersymmetric gauged quantum mechanics on $\mathbb{I}$, are equivalent to a zero-dimensional gauged sigma model with target $L$ and gauge group $G_{\mathbb{C}}$. The path integral for this zero-dimensional theory takes the same form as the formula (2.93), but with $L$ replaced by $\mathcal{L}$ and $W-W_{0}$ understood as a function on $\mathcal{L}$.

\section{$5 \quad$ Gauge theory applications}

In the final section, we discuss examples of A-type and B-type constructions which realize gauge theories of dimension $d=1$ to 6 . The theories constructed here are gauged quantum mechanics, gauged symplectic bosons, and Chern-Simons theory and its higherdimensional variants.

\subsection{Gauged quantum mechanics}

Let us begin with $d=1$. This example is a generalization of the example studied in section 3, and relates the $\Omega$-deformed gauged Rozansky-Witten theory, the gauged Amodel and gauged quantum mechanics. We will restrict ourselves to the case when the target spaces are hyperkähler.

The target space of gauged Rozansky-Witten theory [53] with gauge group $H$ is a

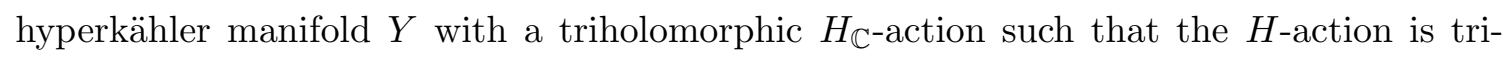
Hamiltonian. Corresponding to the three complex structures $I, J, K$ of $Y$, there are three symplectic structures $\omega_{I}, \omega_{J}, \omega_{K}$ and three moment maps $\mu_{I}, \mu_{J}, \mu_{K}$.

Placed on $\mathbb{D} \times \mathbb{M}_{1}$, gauged Rozansky-Witten theory can be formulated as a gauged B-model on $\mathbb{D}$. The B-model description singles out one of the complex structures of $Y$, which we take to be $I$.

In the B-model description, we have a chiral multiplet whose scalar field takes values in $Y$, as well as a chiral multiplet whose scalar field is the component $\mathcal{A}_{t}$ of a complex linear combination of a three-dimensional gauge field and a $\mathfrak{h}$-valued one-form field. Hence, the target space of the gauged B-model is

$$
X=\operatorname{Map}\left(\mathbb{M}_{1}, Y \times \mathfrak{h}_{\mathbb{C}}\right)
$$


The gauge group is

$$
G=\operatorname{Map}\left(\mathbb{M}_{1}, H\right),
$$

acting on $X$ by the pointwise $H$-action on $Y$ and $\mathfrak{h}_{\mathbb{C}}$. This is the group of gauge transformations for a gauge theory on $\mathbb{M}_{1}$ with gauge group $H$. (More precisely, these are local descriptions of $X$ and $G$ if the gauge bundle for gauged Rozansky-Witten theory is nontrivial.)

The superpotential is the gauge invariant version of the functional (3.19). For simplicity, let us assume that the holomorphic symplectic form $\Omega=\omega_{J}+\mathrm{i} \omega_{K}$ for $I$ can be written as $\Omega=\mathrm{d} \Lambda$ for some $H$-invariant holomorphic one-form $\Lambda$. Using $\Lambda$, we can define $\mu_{J}$ and $\mu_{K}$ by $\left(\mu_{J}+\mathrm{i} \mu_{K}\right)_{a}=-\iota_{v_{a}} \Lambda$ since $\mathrm{d}\left(\mu_{J}+\mathrm{i} \mu_{K}\right)_{a}=-\left(\mathcal{L}_{v_{a}}-\iota_{v_{a}} \mathrm{~d}\right) \Lambda=\iota_{v_{a}} \Omega$. Then,

$$
W=\frac{\mathrm{i}}{2} \int_{\mathbb{M}_{1}}\left(\varphi^{*} \Lambda-\mathcal{A}^{a}\left(\mu_{J}+\mathrm{i} \mu_{K}\right)_{a}\right)
$$

is the gauge invariant superpotential.

We apply the $\Omega$-deformation to this gauged B-model and perform cigar reduction. Then, we obtain supersymmetric gauged quantum mechanics on $\mathbb{I}$ with target $X$, and this is equivalent to the gauged A-model [42] on $\mathbb{I} \times \mathbb{M}_{1}$ with target $Y$ and symplectic form $\omega_{K_{\alpha}}=\omega_{K} \cos \alpha-\omega_{J} \sin \alpha$. A generalization of the canonical coisotropic brane appears at

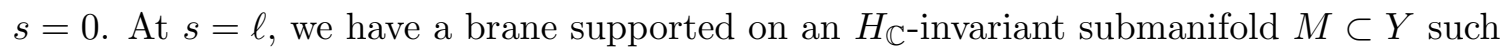
that its image in $Y / / H$ is Lagrangian. This brane comes from a brane in the $\Omega$-deformed gauged B-model whose support is $L=\operatorname{Map}\left(\mathbb{M}_{1}, M\right)$.

Finally, shrinking $\mathbb{I}$ to a point, we get a zero-dimensional gauged sigma model with target $L$ and gauge group $G_{\mathbb{C}}$. The action of the model is given by $W$. This is gauged quantum mechanics on $\mathbb{M}_{1}$ with target $M$ and gauge group $H_{\mathbb{C}}$.

Integrating over $\mathcal{A}$ sets

$$
\mu_{J}+i \mu_{K}=0
$$

so this gauged quantum mechanics may also be regarded as nongauged quantum mechanics with target space

$$
\left(M \cap\left(\mu_{J}+\mathrm{i} \mu_{K}\right)^{-1}(0)\right) / H_{\mathbb{C}},
$$

which is a submanifold of the hyperkähler quotient

$$
Y / / H=\left(\mu_{I}^{-1}(0) \cap \mu_{J}^{-1}(0) \cap \mu_{K}^{-1}(0)\right) / H \cong\left(\mu_{J}+\mathrm{i} \mu_{K}\right)^{-1}(0) / H_{\mathbb{C}} .
$$

As we have seen in section 3 , for the whole construction to work we must choose $M$ in such a way that this submanifold of $Y / / / H$ is Lagrangian with respect to $\omega_{I}$ and $\omega_{K_{\alpha}}$, and symplectic with respect to $\omega_{J_{\alpha}}$.

\subsection{Gauged symplectic bosons}

Next, we consider an example with $d=2$. In this example, a chiral CFT on a Riemann surface $\mathbb{M}_{2}$ is realized by an $\mathcal{N}=2$ supersymmetric gauge theory on $\mathbb{D} \times \mathbb{M}_{2}[6,7]$ and by an $\mathcal{N}=4$ supersymmetric gauge theory on $\mathbb{I} \times \mathbb{M}_{2}[3,4]$. 
Let us take a four-dimensional $\mathcal{N}=2$ supersymmetric gauge theory, constructed from a vector multiplet for gauge group $H$ and a hypermultiplet in a complex symplectic representation $Y$ of $H$. The theory has eight supercharges $Q_{\alpha}^{A}, \bar{Q}_{A \dot{\alpha}}$, where $\alpha= \pm, \dot{\alpha}= \pm$ are spinor indices, and $A= \pm$ is an index for an R-symmetry group $\mathrm{SU}(2)_{R}$ under which $Q_{\alpha}^{A}$ and $\bar{Q}_{A \alpha}$ transform in the fundamental and the antifundamental representations, respectively. An R-symmetry group $\mathrm{U}(1)_{r}$ rotates $Q_{\alpha}^{A}$ with charge $r=+\frac{1}{2}$ and $\bar{Q}_{\dot{\alpha}}^{A}$ with $r=-\frac{1}{2}$.

We place the theory on $\mathbb{D} \times \mathbb{M}_{2}$ and apply Kapustin's twist [40]. Under the rotation group $\mathrm{U}(1)_{\mathbb{D}}$ of $\mathbb{D}$, the supercharges $Q_{+}^{A}, \bar{Q}_{-}^{A}$ have spin $M_{\mathbb{D}}=+\frac{1}{2}$ and $Q_{-}^{A}, \bar{Q}_{\dot{+}}^{A}$ have $M_{\mathbb{D}}=-\frac{1}{2}$. Under the rotation group $\mathrm{U}(1)_{\mathbb{M}_{2}}$ of $\mathbb{M}_{2}, Q_{+}^{A}, \bar{Q}_{\dot{+}}^{A}$ have $M_{\mathbb{M}_{2}}=+\frac{1}{2}$ and $Q_{-}^{A}, \bar{Q}_{\dot{-}}^{A}$ have $M_{\mathbb{M}_{2}}=-\frac{1}{2}$. The Kapustin twist replaces the rotation generators $M_{\mathbb{D}}, M_{\mathbb{M}_{2}}$ with the twisted rotation generators ${ }^{6}$

$$
\begin{aligned}
M_{\mathbb{D}}^{\prime} & =M_{\mathbb{D}}+r, \\
M_{\mathbb{M}_{2}}^{\prime} & =M_{\mathbb{M}_{2}}+R,
\end{aligned}
$$

where $R$ is the generator of a subgroup $\mathrm{U}(1)_{R} \subset \mathrm{SU}(2)_{R}$ such that $Q_{\alpha}^{ \pm}, \bar{Q}_{\dot{\alpha}}^{ \pm}$have $R= \pm \frac{1}{2}$.

Since $Q_{-}^{+}, Q_{+}^{-}, \bar{Q}_{-}^{+}$and $\bar{Q}_{\dot{+}}^{-}$have $M_{\mathbb{M}_{2}}^{\prime}=0$, these supercharges become scalars on $\mathbb{M}_{2}$ after the twist, and as such are preserved even when $\mathbb{M}_{2}$ is curved. Two of them have $M_{\mathbb{D}}=+\frac{1}{2}$ and the other two have $M_{\mathbb{D}}=-\frac{1}{2}$, so they generate $\mathcal{N}=(2,2)$ supersymmetry on $\mathbb{D}$. In the language of $\mathcal{N}=(2,2)$ supersymmetry, $\mathrm{U}(1)_{R}$ is called the vector R-symmetry, while $\mathrm{U}(1)_{r}$ is the axial R-symmetry, as can be seen from the fact that $\mathrm{U}(1)_{r}$ rotates the scalars in the vector multiplet but $\mathrm{U}(1)_{R}$ does not.

The replacement of $M_{\mathbb{D}}$ with $M_{\mathbb{D}}^{\prime}$ is the B-twist of $\mathcal{N}=(2,2)$ supersymmetry, which by definition twists the rotation group with the axial R-symmetry. Among the four supercharges, $Q_{-}^{+}$and $\bar{Q}_{-}^{+}$are scalars with respect to $M_{\mathbb{D}}^{\prime}$. The linear combination

$$
Q_{0}=Q_{-}^{+}+\bar{Q}_{-}^{+}
$$

squares to zero, and by taking the $Q_{0}$-cohomology we get a topological theory on $\mathbb{D}$ since the components of the stress tensor along $\mathbb{D}$ are $Q_{0}$-exact. It turns out that the generator of antiholomorphic translations on $\mathbb{M}_{2}$ is also $Q_{0}$-exact, so the $Q_{0}$-cohomology defines a holomorphic-topological theory on $\mathbb{D} \times \mathbb{M}_{2}$, which we will refer to as Kapustin theory.

As a gauged B-model on $\mathbb{D}$, Kapustin theory has a chiral multiplet whose scalar field is valued in $Y$ and another chiral multiplet whose scalar is the component $A_{\bar{z}}$ of the gauge field along the antiholomorphic direction of $\mathbb{M}_{2}$. The target space of the gauged B-model is therefore

$$
X=\operatorname{Map}\left(\mathbb{M}_{2}, Y \times \mathfrak{h}_{\mathbb{C}}\right),
$$

\footnotetext{
${ }^{6}$ The $\mathrm{U}(1)_{r}$ R-symmetry is anomalous if the one-loop beta function is nonzero. Nevertheless, one can make sense of the following construction by introducing to the action a term that breaks the rotation invariance on $\mathbb{D}[6]$. This term is chosen in such a way that the anomaly in $\mathrm{U}(1)_{r}$ cancels the explicit violation of $\mathrm{U}(1)_{\mathbb{D}}$. This is done at the cost of losing gauge invariance, but gauge invariance is restored if an appropriate surface defect is inserted at the center of $\mathbb{D}$. After the reduction to a theory on $\mathbb{I} \times \mathbb{M}_{2}$, the surface defect becomes a boundary theory localized at $s=0$. Such boundary theories are important ingredients in the construction of [4].
} 
and the gauge group is

$$
G=\operatorname{Map}\left(\mathbb{M}_{2}, H\right)
$$

The hypermultiplet consists of a pair of chiral multiplets in conjugate representations. In terms of the scalar fields $q$ and $\tilde{q}$ of these chiral multiplets, the superpotential is given by $[6]$

$$
W=\int_{\mathbb{M}_{2}} \tilde{q} \bar{\partial}_{A} q
$$

up to an overall factor which can be absorbed by a rescaling of fields. Note that taking $\mathbb{M}_{2}=$ $\mathbb{M}_{1} \times \mathbb{S}^{1}$ and performing dimensional reduction on $\mathbb{S}^{1}$ reduces $W$ to the superpotential (5.3) for gauged Rozansky-Witten theory.

From this B-model description, we see that if we turn on the $\Omega$-deformation using the rotation symmetry of $\mathbb{D}$, Kapustin theory on $\mathbb{D} \times \mathbb{M}_{2}$ becomes equivalent to a chiral CFT on $\mathbb{M}_{2}$, described by the action $W$. This CFT is known as the system of gauged symplectic bosons with values in $Y$. The integration cycle for the path integral is specified by a Lagrangian brane placed on $\partial \mathbb{D}$.

Reducing the $\Omega$-deformed Kapustin theory on the circle fibers of the cigar $\mathbb{D}$, we arrive at the corresponding A-type theory on $\mathbb{I} \times \mathbb{M}_{2}$. By construction, this is a holomorphictopological theory on $\mathbb{I} \times \mathbb{M}_{2}$. As we will argue shortly, it is a certain twist of the circle reduction of the parent four-dimensional $\mathcal{N}=2$ supersymmetric gauge theory, which is a twist of a three-dimensional $\mathcal{N}=4$ supersymmetric gauge theory. This is a nontrivial statement as one might have expected that the $\Omega$-deformation would affect the reduced theory. For the moment let us accept this statement as a fact, and identify the relevant twist.

A three-dimensional $\mathcal{N}=4$ supersymmetric gauge theory has an R-symmetry group $\mathrm{SU}(2)_{H} \times \mathrm{SU}(2)_{C}$ and eight supercharges $Q_{\alpha}^{A \dot{A}}$, where $\alpha= \pm$ is a spinor index, $A= \pm$ is an $\mathrm{SU}(2)_{H}$ index and $\dot{A}=\dot{ \pm}$ is an $\mathrm{SU}(2)_{C}$ index. If the theory is constructed by reduction from four dimensions, $\mathrm{SU}(2)_{H}$ comes from $\mathrm{SU}(2)_{R}$, whereas $\mathrm{U}(1)_{r}$ becomes a subgroup $\mathrm{U}(1)_{C} \subset \mathrm{SU}(2)_{C}$. In the case at hand, the supercharges are identified as $Q_{\alpha}^{A \dot{+}}=Q_{\alpha}^{A}$ and $Q_{\alpha}^{A \dot{-}}=\bar{Q}_{\dot{\alpha}}^{A}$.

Formulated on $\mathbb{I} \times \mathbb{M}_{2}$, the theory admits two distinct twists [54]. One may twist it by replacing the rotation group $U(1)_{\mathbb{M}_{2}}$ with the diagonal subgroup of $U(1)_{\mathbb{M}_{2}} \times U(1)_{C}$. This is called the C-twist. According to the definition (5.8) of the twisted rotation generator $M_{\mathbb{M}_{2}}^{\prime}$, relevant for the A-type construction is the H-twist, which twists $\mathrm{U}(1)_{\mathbb{M}_{2}}$ with a subgroup $\mathrm{U}(1)_{H} \subset \mathrm{SU}(2)_{H}$.

The H-twist makes four supercharges scalars on $\mathbb{M}_{2}$. To see what linear combination of these should be used for the construction of the holomorphic-topological theory, let us take $\mathbb{M}_{2}=\mathbb{C}$ and consider placing Kapustin theory on $\mathbb{I} \times \mathbb{S}^{1} \times \mathbb{C}$. We think of $\mathbb{I} \times \mathbb{S}^{1}$ as modeling the flat cylinder part of $\mathbb{D}$.

On this spacetime all supercharges are unbroken, and the Lie derivative by $V=e^{\mathrm{i} \alpha} \partial_{\hat{\theta}}$ acts on differential forms simply by acting on their coefficient functions with $V$. Then, the 
$\Omega$-deformed supercharge $Q_{V}$ can be written, up to an overall factor, as ${ }^{7}$

$$
Q_{V}=Q_{-}^{+}+\bar{Q}_{-}^{+}+e^{\mathrm{i} \alpha}\left(Q_{+}^{-}-\bar{Q}_{\dot{+}}^{-}\right),
$$

because this linear combination of supercharges satisfies $Q_{V}^{2} \propto \mathcal{L}_{V}$. Upon dimensional reduction in the $\theta$-direction, $Q_{V}$ descends to the supercharge

$$
Q=Q_{-}^{+\dot{+}}+Q_{-}^{+\dot{-}}+e^{\mathrm{i} \alpha}\left(Q_{+}^{-\dot{+}}-Q_{+}^{-\dot{-}}\right)
$$

in an $\mathcal{N}=4$ supersymmetric gauge theory on $\mathbb{I} \times \mathbb{C}$. This is the supercharge whose cohomology defines the holomorphic-topological theory.

The above argument explains why the $\Omega$-deformation "disappears" when a B-twisted supersymmetric field theory is reduced on a circle, that is, the circle reduction of the $\Omega$ deformed B-twisted theory is a twist of the reduction of the undeformed theory, not a deformation thereof. The reason is that the $\Omega$-deformed supercharge $Q_{V}$ already exists in the undeformed theory, and becomes the supercharge $Q$ for the twisted theory obtained by the reduction. The only thing the $\Omega$-deformation can do is to change the action by $Q_{V}$-invariant terms, which merely corresponds to a $Q$-invariant deformation of the reduced theory. We have fixed the action for the reduced theory by asking it to have the standard bosonic terms (4.69).

In fact, we can characterize the $Q$-cohomology using only a half of $Q$, and it is illuminating to do so. This characterization is based on the fact that the $Q_{V}$-cohomology of operators is isomorphic to the $Q_{V}$-cohomology of states by conformal invariance (which the $Q_{V}$-cohomology possesses), and by unitarity the latter is isomorphic to the space of $Q_{V}$-harmonic states. If we define

$$
\begin{aligned}
& \mathscr{Q}_{V}=Q_{-}^{+}+e^{\mathrm{i} \alpha} Q_{+}^{-}, \\
& \bar{Q}_{V}=\bar{Q}_{-}^{+}-e^{\mathrm{i} \alpha} \bar{Q}_{\dot{+}}^{-},
\end{aligned}
$$

then $Q_{V}=\phi_{V}+\bar{\phi}_{V}$ and

$$
\left\{Q_{V}, Q_{V}^{\dagger}\right\}=2\left\{\phi_{V}, \phi_{V}^{\dagger}\right\}=2\left\{\bar{\phi}_{V}, \bar{\phi}_{V}^{\dagger}\right\}
$$

where the hermiticity condition is given by $\left(Q_{\alpha}^{+}\right)^{\dagger}=-\bar{Q}_{\dot{\alpha}}^{-}$and $\left(Q_{\alpha}^{-}\right)^{\dagger}=\bar{Q}_{\dot{\alpha}}^{+}$. Therefore, the $Q_{V}$-cohomology is isomorphic to both $\phi_{V}$-cohomology and $\bar{\phi}_{V^{-}}$-cohomology.

Let us choose $\phi_{V}$. Under the dimensional reduction this supercharge becomes

$$
\phi=Q_{-}^{+\dot{+}}+e^{\mathrm{i} \alpha} Q_{+}^{-\dot{+}}
$$

and the $Q$-cohomology is isomorphic to the $Q$-cohomology. The supercharge $\not$ is used in the discussions of holomorphic boundary conditions in $[3,4,54]$ (up to a flip of the spinor index).

\footnotetext{
${ }^{7}$ The supersymmetry transformation laws for the $\Omega$-deformed gauged B-model may be obtained from those in [55] by substitution $\partial_{0}=\mathrm{i} \partial_{\hat{\theta}}, \partial_{1}=\partial_{\hat{s}}$ and $\bar{\epsilon}_{ \pm}= \pm \mathrm{i} / 2, \epsilon_{ \pm}=-\left(V^{\hat{s}} \pm \mathrm{i} V^{\hat{\theta}}\right) / 2$. This gives $Q_{V} \propto$ $\bar{Q}_{+}+\bar{Q}_{-}+\left(V^{\hat{\theta}}-\mathrm{i} V^{\hat{s}}\right) Q_{-}+\left(V^{\hat{\theta}}+\mathrm{i} V^{\hat{s}}\right) Q_{+}$. According to [6], this supercharge is identified with $Q_{-}^{+}+\bar{Q}_{-}^{+}+$ $\left(V^{\hat{\theta}}-\mathrm{i} V^{\hat{s}}\right) Q_{+}^{-}-\left(V^{\hat{\theta}}+\mathrm{i} V^{\hat{s}}\right) \bar{Q}_{\dot{+}}^{-}$in the Kapustin twist.
} 
There is something remarkable about $\varnothing$ : it is proportional to a $\mathrm{U}(1)_{H}$ rotation of the supercharge $Q_{-}^{+\dot{+}}+Q_{+}^{-\dot{+}}$ for the A-twist [33], in which the rotation group $\mathrm{SU}(2)$ is replaced by the (anti)diagonal subgroup of $\mathrm{SU}(2) \times \mathrm{SU}(2)_{H}$. Consequently, the $Q$-cohomology of the twisted theory on $\mathbb{I} \times \mathbb{M}_{2}$ is actually fully topological in the bulk, not topological on $\mathbb{I}$ and holomorphic on $\mathbb{M}_{2}$ as one might have expected.

This is consistent with the relation between gauged Rozansky-Witten theory and the gauged A-model, discussed in section 5.1. If we take $\mathbb{M}_{2}=\mathbb{M}_{1} \times \mathbb{S}^{1}$ and perform reduction on $\mathbb{S}^{1}$, the supercharge $Q_{-}^{+}+\bar{Q}_{-}^{+}$for Kapustin theory on $\mathbb{D} \times \mathbb{M}_{2}$ reduces to the supercharge $Q_{-}^{+\dot{+}}+Q_{+}^{+\dot{-}}$ for an $\mathcal{N}=4$ supersymmetric gauge theory on $\mathbb{D} \times \mathbb{M}_{1}$. This is the supercharge for the B-twist, so Kapustin theory reduces to gauged Rozansky-Witten theory. The cigar reduction of the $\Omega$-deformed gauged Rozansky-Witten theory on $\mathbb{D} \times \mathbb{M}_{1}$ is the gauged A-model on $\mathbb{I} \times \mathbb{M}_{1}$, which has two-dimensional topological invariance, not just topological on $\mathbb{I}$ and on $\mathbb{M}_{1}$ separately.

At first sight, it might be puzzling that one obtains something holomorphic like a chiral CFT out of a fully topological theory. The resolution to this puzzle is that the threedimensional topological invariance is broken at the center of $\mathbb{D}$ where $Q_{V}$ reduces to $Q_{-}^{+}$. On the boundary at $s=0$, boundary conditions allow for additional holomorphic observables.

Thus, we have found that the A-type theory on $\mathbb{I} \times \mathbb{M}_{2}$ that realizes the gauged symplectic bosons with values in $Y$ is the A-twist of an $\mathcal{N}=4$ supersymmetric gauge theory. This theory has a vector multiplet for gauge group $H$ and a hypermultiplet valued in $Y$, originating from their counterparts in four dimensions. The vector multiplet has three scalar fields transforming as a triplet under $\mathrm{SU}(2)_{C}$. They come from the complex scalar in the four-dimensional $\mathcal{N}=2$ vector multiplet and the component $A_{\theta}$ of the four-dimensional gauge field. The hypermultiplet contains a pair of scalar fields $q^{A}$, transforming as a double under $\mathrm{SU}(2)_{H}$. In terms of the four-dimensional fields, $q^{+}=q$ and $q^{-}=\tilde{q}^{\dagger}$.

Let us determine the boundary conditions on these fields at $s=0$.

If we describe the theory as supersymmetric gauged quantum mechanics on $\mathbb{I}$ with target $X$ and gauge group $G$, from the three-dimensional vector multiplet we get a onedimensional vector multiplet and a one-dimensional $\mathfrak{g}$-valued chiral multiplet whose scalar field is $A_{\bar{z}}$. At $s=0$, all bosonic fields of the one-dimensional vector multiplet are set to zero, while $A_{\bar{z}}$ satisfies the gradient flow equation (2.87). In the three-dimensional terms, these conditions amount to the Dirichlet condition on the vector multiplet scalars and a deformed Neumann condition

$$
F_{\hat{s} \bar{z}} \propto e^{\mathrm{i} \alpha}\left(\left(q^{+}\right)^{\dagger} T^{a} q^{-}\right) T_{a}
$$

on the gauge field.

The three-dimensional hypermultiplet gives rise to a pair of chiral multiplets in supersymmetric gauged quantum mechanics. At $s=0$, the boundary condition for the scalar fields of these multiplets demands

$$
\partial_{\hat{s}} q^{-} \propto e^{-\mathrm{i} \alpha} \bar{\partial}_{A} q^{+} .
$$


The boundary conditions just described are consistent with those discussed in $[3,4]$, where it was found that symplectic bosons arise from boundaries in this three-dimensional $\mathcal{N}=4$ supersymmetric gauge theory.

\subsection{Chern-Simons theory}

Now we discuss an example with $d=3$. In this example, Chern-Simons theory on a threemanifold $\mathbb{M}_{3}$ is realized by maximally supersymmetric Yang-Mills theories on $\mathbb{D} \times \mathbb{M}_{3}[18]$ and $\mathbb{I} \times \mathbb{M}_{3}[8,9]$.

Five-dimensional $\mathcal{N}=2$ super Yang-Mills theory, like any other maximally supersymmetric Yang-Mills theories, can be constructed from ten-dimensional super Yang-Mills theory by dimensional reduction. Let $\left(x^{1}, \ldots, x^{10}\right)$ be coordinates on $\mathbb{R}^{10}$, and reduce super Yang-Mills theory on $\mathbb{R}^{10}$ in the directions of $x^{6}, \ldots, x^{10}$. Then, the components $A_{6}, \ldots$, $A_{10}$ of the ten-dimensional gauge field are turned into five scalar fields $\phi_{6}, \ldots, \phi_{10}$, valued in the Lie algebra $\mathfrak{h}$ of the gauge group $H$. They transform in the vector representation of the R-symmetry group $\operatorname{Spin}(5)_{R}$, which originates from the rotation symmetry of $\mathbb{R}^{5}$ on which the reduction is performed. The sixteen supercharges of the theory transform in the spinor representation of $\operatorname{Spin}(5)_{R}$.

We place this theory on $\mathbb{D} \times \mathbb{M}_{3}$, and twist the rotation group $\mathrm{SU}(2)_{\mathbb{M}_{3}}$ on $\mathbb{M}_{3}$ by the subgroup $\mathrm{SU}(2)_{R}$ of $\operatorname{Spin}(5)_{R}$ rotating $\phi_{8}, \phi_{9}$ and $\phi_{10}$. The supercharges transform as doublets under $\mathrm{SU}(2)_{\mathbb{M}_{3}}$ and $\mathrm{SU}(2)_{R}$, so a quarter of the sixteen supercharges become singlets under the diagonal subgroup of $\mathrm{SU}(2)_{\mathbb{M}_{3}} \times \mathrm{SU}(2)_{R}$. These four supercharges are scalars under the twisted rotation group on $\mathbb{M}_{3}$, and can be preserved even when $\mathbb{M}_{3}$ is curved. They have spins $\pm \frac{1}{2}$ under $\mathrm{U}(1)_{\mathbb{D}}$, so generate $\mathcal{N}=(2,2)$ supersymmetry on $\mathbb{D}$.

Let $\mathrm{U}(1)_{r}$ be the subgroup of $\operatorname{Spin}(5)_{R}$ rotating $\phi_{6}$ and $\phi_{7}$. From the point of view of $\mathcal{N}=(2,2)$ supersymmetry, $\mathrm{U}(1)_{r}$ is the axial R-symmetry rotating the two scalars in the vector multiplet. Further twisting the theory on $\mathbb{D}$ with $\mathrm{U}(1)_{r}$, we get a gauged B-model.

In the twisted theory, $\phi_{8}, \phi_{9}, \phi_{10}$ are the components of a one-form on $\mathbb{M}_{3}$. They combine with $A_{3}, A_{4}, A_{5}$ to form a $Q$-invariant complex gauge field $\mathcal{A}$ on $\mathbb{M}_{3}$ :

$$
\mathcal{A}=\left(A_{3}+\mathrm{i} \phi_{8}\right) \mathrm{d} x^{3}+\left(A_{4}+\mathrm{i} \phi_{9}\right) \mathrm{d} x^{4}+\left(A_{5}+\mathrm{i} \phi_{10}\right) \mathrm{d} x^{5} .
$$

The components of $\mathcal{A}$ are the scalar fields of three chiral multiplets. Hence, the target space $X$ of the gauged B-model is the space of complex gauge fields on $\mathbb{M}_{3}$. The gauge group $G$ is the group of gauge transformations on $\mathbb{M}_{3}$, and $G_{\mathbb{C}}$ acts naturally on $X$ by complex gauge transformations. If the gauge bundle is trivial, we have

$$
X=\Omega^{1}\left(\mathbb{M}_{3}, \mathfrak{h}_{\mathbb{C}}\right)
$$

and

$$
G=\operatorname{Map}\left(\mathbb{M}_{3}, H\right) .
$$

The $G$-invariant Kähler metric on $X$ is given by

$$
g(v, w)=-\frac{1}{2 g_{5 \mathrm{~d}}^{2}} \int_{\mathbb{M}_{3}} \operatorname{Tr}(v \wedge \star \bar{w}+\bar{v} \wedge \star w),
$$




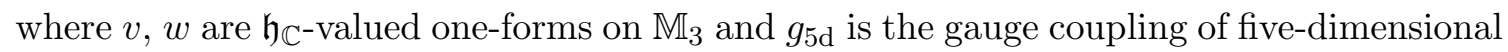
$\mathcal{N}=2$ super Yang-Mills theory.

The superpotential $W$ is a gauge invariant functional, given by the integral of a holomorphic function of $\mathcal{A}$ over $\mathbb{M}_{3}$. For the total action to be second order in derivatives after the auxiliary fields are integrated out, $W$ must be first order. In view of these requirements, the only candidate for $W$ is the integral of the Chern-Simons form

$$
\operatorname{CS}(\mathcal{A})=\operatorname{Tr}\left(\mathcal{A} \wedge \mathrm{d} \mathcal{A}+\frac{2}{3} \mathcal{A} \wedge \mathcal{A} \wedge \mathcal{A}\right)
$$

over $\mathbb{M}_{3}$, up to an overall normalization factor. The overall factor is meaningful in this example since $W$ is not homogeneous and cannot be absorbed by a rescaling of $\mathcal{A}$. The inhomogeneity of $W$ also explains why the vector R-symmetry is absent in the B-model description.

The absolute value of the normalization of $W$ is fixed by the requirement that integrating out the auxiliary fields reproduces the kinetic term

$$
-\frac{1}{4 g_{5 \mathrm{~d}}^{2}} \int_{\mathbb{D} \times \mathbb{M}_{3}} \operatorname{Tr}(F \wedge \star F)
$$

for the five-dimensional gauge field. This is satisfied if we take

$$
W=\frac{1}{4 g_{5 \mathrm{~d}}^{2}} \int_{\mathbb{M}_{3}} \operatorname{CS}(\mathcal{A}) .
$$

The phase of $W$ is not so important because shifting the phase has the same effect as the action of the broken vector R-rotation that leaves $\mathcal{A}$ intact. Different choices of the phase correspond to different ways to identify the supercharge of the twisted five-dimensional theory and that of the gauged B-model.

From this superpotential, we learn that the $\Omega$-deformation of the twisted $\mathcal{N}=2$ super Yang-Mills theory on $\mathbb{D} \times \mathbb{M}_{3}$ with gauge group $H$ is Chern-Simons theory with gauge group $H_{\mathbb{C}}$, described by the action

$$
-\frac{\pi \mathrm{i}}{2 \epsilon g_{5 \mathrm{~d}}^{2}} \int_{\mathbb{M}_{3}} \operatorname{CS}(\mathcal{A})
$$

Let us turn to the corresponding A-type construction. The A-type theory is the reduction of the above theory in the direction of $x^{2}=\theta$. This is a topological twist of $\mathcal{N}=4$ super Yang-Mills theory on $\mathbb{I} \times \mathbb{M}_{3}$, with gauge coupling

$$
g_{4 \mathrm{~d}}=\sqrt{\frac{|\epsilon|}{2 \pi}} g_{5 \mathrm{~d}} .
$$

As we now argue, the relevant twist is the GL-twist [34-36], with its $\mathbb{C P}^{1}$-valued parameter equal to

$$
t=\frac{1-\cos \alpha}{\sin \alpha} .
$$

The GL-twist of $\mathcal{N}=4$ super Yang-Mills theory on a four-manifold replaces the rotation group $\operatorname{Spin}(4)$ with the diagonal subgroup of $\operatorname{Spin}(4) \times \operatorname{Spin}(4)_{R}$, where the second 
factor is a subgroup of the R-symmetry group $\operatorname{Spin}(6)_{R}$. In the present notation, $\operatorname{Spin}(6)_{R}$ rotates $\phi_{6}, \ldots, \phi_{10}$ and $\phi_{2}$ which comes from $A_{2}$, and $\operatorname{Spin}(4)_{R}$ rotates $\phi_{6}, \phi_{8}, \phi_{9}, \phi_{10}$. Thea twist turns the latter four scalars into the components of the one-form

$$
\phi=\phi_{6} \mathrm{~d} x^{1}+\phi_{8} \mathrm{~d} x^{3}+\phi_{9} \mathrm{~d} x^{4}+\phi_{10} \mathrm{~d} x^{5} .
$$

(The coordinate on $\mathbb{I}$ is $x^{1}=s$ and those on $\mathbb{M}_{3}$ are $\left(x^{3}, x^{4}, x^{5}\right)$.)

The GL-twisted theory has two scalar supercharges $Q_{\ell}$ and $Q_{r}$, and one picks a linear combination

$$
Q=Q_{\ell}+t Q_{r}
$$

to define the topological theory, where $t$ takes values in $\mathbb{C P}^{1}$. The supersymmetry transformations generated by $Q$ may be found in [36]. What is important to us is that there is a two-form fermion $\chi$ whose supersymmetry variation is given by

$$
\begin{aligned}
& \delta \chi^{+}=(F-\phi \wedge \phi)^{+}+t\left(\mathrm{~d}_{A} \phi\right)^{+}, \\
& \delta \chi^{-}=t(F-\phi \wedge \phi)^{-}-\left(\mathrm{d}_{A} \phi\right)^{-},
\end{aligned}
$$

where the superscripts + and - denote the self-dual and anti-self-dual parts, respectively.

We claim that when $t$ is real, the GL-twisted $\mathcal{N}=4$ super Yang-Mills theory on $\mathbb{I} \times \mathbb{M}_{3}$ can be reformulated as supersymmetric gauged quantum mechanics on $\mathbb{I}$ with target $X$ and gauge group $G$, with the identification

$$
\sigma_{s}=\phi_{6}
$$

Furthermore, the potential $h$ is determined by the superpotential (5.27) via the relation (2.64), which shows that the theory is the cigar reduction of the $\Omega$-deformed B-model described above.

One way to see this is to compare the supersymmetry variations of fermions that give rise to flow equations. If we make the complex gauge field $\mathcal{A}=\left(A_{s}+\mathrm{i} \sigma_{s}\right) \mathrm{d} s+\mathcal{A}_{3} \mathrm{~d} x^{3}+$ $\mathcal{A}_{4} \mathrm{~d} x^{4}+\mathcal{A}_{5} \mathrm{~d} x^{5}$ and let $\mathcal{F}$ be the curvature of $\mathcal{A}$, then we can write the supersymmetry variation (4.72) as

$$
\delta \chi_{\hat{s}}=\iota_{\partial_{\hat{s}}} \mathcal{F}+e^{\mathrm{i} \alpha} \star_{\mathbb{M}_{3}} \overline{\mathcal{F}},
$$

where we consider $\chi_{\hat{s}}$ as a one-form $\chi_{\hat{s}}^{3} \mathrm{~d} x^{3}+\chi_{\hat{s}}^{4} \mathrm{~d} x^{4}+\chi_{\hat{s}}^{5} \mathrm{~d} x^{5}$. This is equivalent to the supersymmetry variations (5.33) and (5.34), with $t$ given by the formula (5.30).

Another way to argue for this claim is to consider compactification to two dimensions.

Five-dimensional $\mathcal{N}=2$ super Yang-Mills theory has a unique full twist, which replaces the rotation group $\operatorname{Spin}(5)$ with the diagonal subgroup of $\operatorname{Spin}(5) \times \operatorname{Spin}(5)_{R}$. The topological twist we have performed is compatible with this twist. If we take $\mathbb{M}_{3}=\mathbb{R} \times \mathbb{M}_{2}$ for some compact Riemann surface $\mathbb{M}_{2}$, then in the infrared, we get a fully twisted $\mathcal{N}=4$ supersymmetric sigma model on $\mathbb{D} \times \mathbb{R}$ with a hyperkähler target space. (Twisting along $\mathbb{M}_{2}$ breaks half of supersymmetry.) This is Rozansky-Witten theory, and its target space is the moduli space $\mathcal{M}_{H}$ of the Hitchin equations on $\mathbb{M}_{2}$ [56]. The $\Omega$-deformation and cigar reduction then produce the A-model on $\mathbb{I} \times \mathbb{R}$ with target $\mathcal{M}_{H}$. 
The GL-twisted $\mathcal{N}=4$ super Yang-Mills theory for real $t$, upon compactification on $\mathbb{M}_{2}$, indeed reduces to the A-model with target $\mathcal{M}_{H}$ [36]. In $\Omega$-deforming Rozansky-Witten theory with target $\mathcal{M}_{H}$, we have picked a complex structure in which the complex gauge field $A+\mathrm{i} \phi$ on $\mathbb{M}_{2}$ defines holomorphic coordinates. If we call it $I$, then according to [36], the complex structure of the A-model thus obtained can be written in terms of the other two complex structures $J, K$ as

$$
\frac{1-t^{2}}{1+t^{2}} K-\frac{2 t}{1+t^{2}} J=K \cos \alpha-J \sin \alpha
$$

This agrees with the relation between the $\Omega$-deformed Rozansky-Witten theory and the A-model which we found in section 3 .

Thus, we conclude that the GL-twisted $\mathcal{N}=4$ super Yang-Mills theory on $\mathbb{I} \times \mathbb{M}_{3}$ with gauge group $H$ is equivalent to Chern-Simons theory on $\mathbb{M}_{3}$ with gauge group $H_{\mathbb{C}}$, assuming that appropriate boundary conditions are imposed. The boundary conditions at $s=0$ are such that when $\mathbb{M}_{3}=\mathbb{R} \times \mathbb{M}_{2}$, they correspond to the canonical coisotropic brane in the A-model with target $\mathcal{M}_{H}$. This conclusion is in accordance with the results obtained in $[1,8,9]$.

\subsection{Higher-dimensional Chern-Simons theories}

There are higher-dimensional analogs of the above A-type and B-type constructions, in which higher-dimensional variants of Chern-Simons theories are realized by maximally supersymmetric Yang-Mills theories in dimensions five and up.

These Chern-Simons theories are all related by dimensional reduction. The one of highest dimension is six-dimensional Chern-Simons theory, also known as holomorphic Chern-Simons theory [47]. This is a holomorphic theory that can be formulated on a Calabi-Yau threefold $\mathbb{M}_{6}$.

Suppose that we have $\mathbb{M}_{6}=\mathbb{M}_{4} \times \mathbb{R} \times \mathbb{S}^{1}$ for some Calabi-Yau twofold $\mathbb{M}_{4}$, and reduce the theory on $\mathbb{S}^{1}$. The holomorphy on the cylinder $\mathbb{R} \times \mathbb{S}^{1}$ implies that the reduced theory is topological on $\mathbb{R}$. What we get is a holomorphic-topological theory, called five-dimensional Chern-Simons theory [48], placed on $\mathbb{M}_{5}=\mathbb{M}_{4} \times \mathbb{M}_{1}$ with $\mathbb{M}_{1}=\mathbb{R}$.

If we further take $\mathbb{M}_{4}=\mathbb{M}_{2} \times \mathbb{R} \times \mathbb{S}^{1}$ and perform reduction on $\mathbb{S}^{1}$, we get fourdimensional Chern-Simons theory [26-28] on $\mathbb{M}_{4}=\mathbb{M}_{2} \times \mathbb{M}_{2}^{\prime}$ with $\mathbb{M}_{2}^{\prime}=\mathbb{R} \times \mathbb{M}_{1}$, which is holomorphic on $\mathbb{M}_{2}$ and topological on $\mathbb{M}_{2}^{\prime}$.

Finally, taking $\mathbb{M}_{2}=\mathbb{R} \times \mathbb{S}^{1}$ and reducing on $\mathbb{S}^{1}$, we reach the ordinary, fully topological, Chern-Simons theory on $\mathbb{M}_{3}=\mathbb{R} \times \mathbb{M}_{2}^{\prime}$.

Since six-dimensional Chern-Simons theory sits at the top of the hierarchy, let us begin by discussing the constructions of this theory. We will be concise here; some of the assertions made below are explained in more detail in appendix A.

The B-type construction uses eight-dimensional super Yang-Mills theory on $\mathbb{D} \times \mathbb{M}_{6}$. This is made topological on $\mathbb{D}$ by twisting of the rotation group $\mathrm{U}(1)_{\mathbb{D}}$ with the R-symmetry $\mathrm{U}(1)_{R}$. The need for a $\mathrm{U}(1)$ R-symmetry with which the theory is twisted along $\mathbb{D}$ explains why one cannot go higher than eight dimensions in the B-type constructions. 
Covariantly constant spinors on $\mathbb{D} \times \mathbb{M}_{6}$ are linear combinations of the products of covariantly constant spinors on $\mathbb{D}$ and those on $\mathbb{M}_{6}$. The Calabi-Yau threefold $\mathbb{M}_{6}$ has two covariantly constant spinors, one for each chirality. If $\mathbb{D}$ is flat, there are also two on $\mathbb{D}$. Accordingly, the theory has four supercharges when $\mathbb{D}$ is flat. Under $\mathrm{U}(1)_{\mathbb{D}} \times \mathrm{U}(1)_{R}$, two of these supercharges have positive chirality on $\mathbb{M}_{6}$ and weights $\left( \pm \frac{1}{2}, \pm \frac{1}{2}\right)$. The other two have negative chirality on $\mathbb{M}_{6}$ and weights $\left( \pm \frac{1}{2}, \mp \frac{1}{2}\right)$. (The supercharges of ten-dimensional super Yang-Mills theory are spinors of positive chirality.) These supercharges generate $\mathcal{N}=(2,2)$ supersymmetry on $\mathbb{D}$. As $\mathrm{U}(1)_{R}$ rotates the scalars of the $\mathcal{N}=(2,2)$ vector multiplet, $\mathrm{U}(1)_{R}$ is the axial R-symmetry and the twist on $\mathbb{D}$ is the B-twist.

As a gauged B-model, the theory has three chiral multiplets whose scalar fields are the antiholomorphic components $A_{\overline{3}}, A_{\overline{4}}, A_{\overline{5}}$ of the gauge field with respect to local coordinates $\left(z^{3}, z^{4}, z^{5}\right)$ on $\mathbb{M}_{6}$. These scalars make up a $(0,1)$-form

$$
\mathcal{A}=A_{\overline{3}} \mathrm{~d} \bar{z}^{\overline{3}}+A_{\overline{4}} \mathrm{~d} \bar{z}^{\overline{4}}+A_{\overline{5}} \mathrm{~d} \bar{z}^{\overline{5}}
$$

on $\mathbb{M}_{6}$ with values in $\mathfrak{h}_{\mathbb{C}}$, so the target space of the gauged B-model is

$$
X=\Omega^{0,1}\left(\mathbb{M}_{6}, \mathfrak{h}_{\mathbb{C}}\right) .
$$

The gauge group is

$$
G=\operatorname{Map}\left(\mathbb{M}_{6}, H\right),
$$

and $G_{\mathbb{C}}$ acts on $X$ by complex gauge transformations. The $G$-invariant Kähler metric is given by

$$
g(v, w)=-\frac{1}{2 g_{8 \mathrm{~d}}^{2}} \int_{\mathbb{M}_{6}} \operatorname{Tr}(v \wedge \star \bar{w}+\bar{v} \wedge \star w)
$$

for $\mathfrak{h}_{\mathbb{C}^{-v a l u e d}}(0,1)$-forms $v, w$ on $\mathbb{M}_{6}$, where $g_{8 \mathrm{~d}}$ is the gauge coupling.

This example may be thought of as the complexification of the B-type construction of Chern-Simons theory discussed in section 5.3, in the sense that the real coordinates on $\mathbb{M}_{3}$ in that example are replaced by complex coordinates on $\mathbb{M}_{6}$ here. Correspondingly, the superpotential is essentially given by the Chern-Simons functional constructed from $\mathcal{A}$. The Chern-Simons form is a three-form, so to be integrated over $\mathbb{M}_{6}$, it must be wedged with the holomorphic volume form $\Omega_{3}$ of $\mathbb{M}_{6}$ :

$$
W=\frac{2 \mathrm{i}}{g_{8 \mathrm{~d}}^{2}} \int_{\mathbb{M}_{6}} \Omega_{3} \wedge \mathrm{CS}(\mathcal{A})
$$

Here we have normalized $\Omega_{3}$ in such a way that $\Omega_{3} \wedge \bar{\Omega}_{3}$ equals $-\mathrm{i} / 8$ times the volume form of $\mathbb{M}_{6}$. The overall normalization is explained in appendix A.

The above superpotential is the action for holomorphic Chern-Simons theory. Therefore, the $\Omega$-deformation of eight-dimensional super Yang-Mills theory on $\mathbb{D} \times \mathbb{M}_{6}$ is holomorphic Chern-Simons theory on $\mathbb{M}_{6}$.

The corresponding A-type construction is based on seven-dimensional super Yang-Mills theory on $\mathbb{I} \times \mathbb{M}_{6}$. The theory may be reformulated as supersymmetric gauged quantum mechanics on $\mathbb{I}$ with target $X$ and gauge group $G$, and in this description we know what the boundary conditions should be. 
Now we consider dimensional reductions to five- and four-dimensional Chern-Simons theories.

First, let us take $\mathbb{M}_{6}=\mathbb{M}_{4} \times \mathbb{R} \times \mathbb{S}^{1}$ and reduce the theory on $\mathbb{S}^{1}$ to get super Yang-Mills theory on $\mathbb{D} \times \mathbb{M}_{4} \times \mathbb{R}$. If we write $z^{3}=\left(x^{3}-\mathrm{i} x^{8}\right) / 2, z^{4}=\left(x^{4}-\mathrm{i} x^{9}\right) / 2$ and $z^{5}=\left(x^{5}-\mathrm{i} x^{10}\right) / 2$, the superpotential becomes

$$
W=\frac{1}{g_{7 \mathrm{~d}}^{2}} \int_{\mathbb{M}_{4} \times \mathbb{R}} \Omega_{2} \wedge \mathrm{CS}(\mathcal{A})
$$

where $\Omega_{2}$ is the holomorphic volume form of the Calabi-Yau twofold $\mathbb{M}_{4}$, defined by $\Omega_{3}=$ $\Omega_{2} \wedge \mathrm{d} z_{3}$, and

$$
\mathcal{A}=A_{\overline{3}} \mathrm{~d} z^{3}+A_{\overline{4}} \mathrm{~d} x^{4}+\left(A_{5}+\mathrm{i} \phi_{10}\right) \mathrm{d} x^{5},
$$

with the scalar $\phi_{10}$ coming from $A_{10}$.

This superpotential is the action for five-dimensional Chern-Simons theory, so the $\Omega$ deformation of seven-dimensional super Yang-Mills theory on $\mathbb{D} \times \mathbb{M}_{4} \times \mathbb{R}$ is five-dimensional Chern-Simons theory on $\mathbb{M}_{4} \times \mathbb{R}$. The A-type construction involves six-dimensional $\mathcal{N}=$ $(1,1)$ super Yang-Mills theory on $\mathbb{I} \times \mathbb{M}_{4} \times \mathbb{R}$.

Taking $\mathbb{M}_{4}=\mathbb{M}_{2} \times \mathbb{R} \times \mathbb{S}^{1}$ and reducing the seven-dimensional theory on $\mathbb{S}^{1}$, we get six-dimensional $\mathcal{N}=(1,1)$ super Yang-Mills theory on $\mathbb{D} \times \mathbb{M}_{2} \times \mathbb{R}^{2}$. The superpotential for the gauged B-model is

$$
W=\frac{\mathrm{i}}{2 g_{6 \mathrm{~d}}^{2}} \int_{\mathbb{M}_{2} \times \mathbb{R}^{2}} \Omega_{1} \wedge \mathrm{CS}(\mathcal{A})
$$

with $\Omega_{2}=\Omega_{1} \wedge \mathrm{d} z^{4}$ and

$$
\mathcal{A}=A_{\overline{3}} \mathrm{~d} z^{3}+\left(A_{4}+\mathrm{i} \phi_{9}\right) \mathrm{d} x^{4}+\left(A_{5}+\mathrm{i} \phi_{10}\right) \mathrm{d} x^{5} .
$$

With the $\Omega$-deformation turned on, this setup yields four-dimensional Chern-Simons theory on $\mathbb{M}_{2} \times \mathbb{R}^{2}$ [30]. The A-type construction makes use of five-dimensional $\mathcal{N}=2$ super Yang-Mills theory on $\mathbb{I} \times \mathbb{M}_{2} \times \mathbb{R}^{2}$. This setup is essentially the same as the one proposed in $[45,46]$.

Taking $\mathbb{M}_{2}=\mathbb{R} \times \mathbb{S}^{1}$ and reducing these theories on $\mathbb{S}^{1}$, we get back to the A-type and B-type constructions for Chern-Simons theory on $\mathbb{R}^{3}$, described in section 5.3.

One can analyze the above sequence of dimensional reduction and deduce the relevant twist for each of the theories that appear in these constructions. A comprehensive list of twists and the relations between them under dimensional reduction may be found in [57].

The B-type constructions use $(5+n)$-dimensional maximally supersymmetric YangMills theories on $\mathbb{D} \times \mathbb{M}_{2 n} \times \mathbb{R}^{3-n}$. Starting from $n=3$ and going down to $n=2,1$ and 0 , we find that they are twisted along $\mathbb{D} \times \mathbb{R}^{3-n}$ with the $\operatorname{Spin}(5-n)$ R-symmetry. In particular, for $n=0$, we have the fully twisted $\mathcal{N}=2$ super Yang-Mills theory on $\mathbb{D} \times \mathbb{R}^{3}$.

On the A-type side, we have $(4+n)$-dimensional maximally supersymmetric Yang-Mills theories on $\mathbb{I} \times \mathbb{M}_{2 n} \times \mathbb{R}^{3-n}$. As we argued in section 5.3, for $n=0$, we get the GL-twist on $\mathbb{I} \times \mathbb{R}^{3}$ with the parameter $t$ determined by the phase of the $\Omega$-deformation parameter $\epsilon$. Tracing back the sequence of dimensional reduction, we deduce that the theories are twisted along $\mathbb{I} \times \mathbb{R}^{3-n}$ with an $\operatorname{Spin}(4-n)$ subgroup of the R-symmetry group. 


\section{Acknowledgments}

We would like to thank Meer Ashwinkumar for useful correspondences and Dylan Butson for explaining his forthcoming work. NI gratefully acknowledges support from NSF Grant PHY-1911298. The research of JY is supported by the Perimeter Institute for Theoretical Physics. Research at Perimeter Institute is supported in part by the Government of Canada through the Department of Innovation, Science and Economic Development Canada and by the Province of Ontario through the Ministry of Colleges and Universities.

\section{A Eight-dimensional super Yang-Mills theory as $\mathcal{N}=(2,2)$ supersym- metric gauge theory}

In this appendix we formulate eight-dimensional super Yang-Mills theory as an $\mathcal{N}=(2,2)$ supersymmetric gauge theory when part of spacetime is a Calabi-Yau threefold. Since the theory is the dimensional reduction of ten-dimensional super Yang-Mills theory, we will first rewrite the supersymmetry of the latter theory in the form of $\mathcal{N}=(2,2)$ supersymmetry.

To begin with, let us recall spinors in ten dimensions. We work in Euclidean signature.

Spinors in $\mathbb{R}^{10}$ are representations of the Clifford algebra, generated by the gamma matrices $\Gamma_{I}, I=1, \ldots, 10$, satisfying the relations

$$
\left\{\Gamma_{I}, \Gamma_{J}\right\}=2 \delta_{I J}
$$

The chirality operator $-\mathrm{i} \Gamma_{1} \cdots \Gamma_{10}$ has eigenvalues \pm 1 .

Introduce the complex coordinates $z^{i}=x^{2 i-1}+\mathrm{i} x^{2 i}, i=1, \ldots, 5$. Then, the corresponding gamma matrices

$$
\begin{aligned}
\gamma_{i} & =\frac{1}{2}\left(\Gamma_{2 i-1}-\mathrm{i} \Gamma_{2 i}\right), \\
\bar{\gamma}_{i} & =\frac{1}{2}\left(\Gamma_{2 i-1}+\mathrm{i} \Gamma_{2 i}\right)
\end{aligned}
$$

obey the relations

$$
\left\{\gamma_{i}, \bar{\gamma}_{j}\right\}=\delta_{i j}
$$

Thus, one may think of $\bar{\gamma}_{i}$ as fermion creation operators and $\gamma_{i}$ as annihilation operators.

In this language, spinors are states in the fermionic Fock space built on the vacuum by the action of the creation operators. The chirality operator can be expressed in terms of the fermion number operators $\bar{\gamma}_{i} \gamma_{i}$ as

$$
-\mathrm{i} \Gamma_{1} \cdots \Gamma_{10}=\prod_{i=1}^{5}\left(2 \bar{\gamma}_{i} \gamma_{i}-1\right) .
$$

Spinors of positive chirality have an odd number of excitations, and those of negative chirality have an even number of excitations. They have sixteen components.

A concise way of presenting spinors on $\mathbb{C}^{5}$ is to use differential forms. In this presentation, spinors are linear combinations of $(p, 0)$-forms, with $p=0, \ldots, 5$. The action of 
the gamma matrices are operations changing the form degree by one:

$$
\begin{aligned}
& \gamma_{i}=\iota \partial_{i}, \\
& \bar{\gamma}_{i}=\mathrm{d} z^{i} \wedge .
\end{aligned}
$$

Positive chirality spinors and negative chirality ones are odd forms and even forms, respectively. The product $\bar{\alpha} \beta$ of two spinors $\alpha, \beta$ is defined by

$$
\bar{\alpha} \beta=\left(\alpha^{t} \wedge \beta\right)^{\text {top }},
$$

where the transpose acts as

$$
\left(\mathrm{d} z^{i_{1}} \wedge \cdots \wedge \mathrm{d} z^{i_{p}}\right)^{t}=\mathrm{d} z^{i_{p}} \wedge \cdots \wedge \mathrm{d} z^{i_{1}}
$$

and $\alpha^{\text {top }}$ is the top component of $\alpha$.

Now we consider super Yang-Mills theory on $\mathbb{C}^{5}$. The theory has a gauge field $A$ and a spinor field $\Psi$, and supersymmetry transforms them by

$$
\begin{aligned}
\delta A_{I} & =\mathrm{i} \bar{\epsilon} \Gamma_{I} \Psi, \\
\delta \Psi & =\frac{1}{4}\left[\Gamma_{I}, \Gamma_{J}\right] F^{I J} \epsilon .
\end{aligned}
$$

Both the fermion $\Psi$ and the supersymmetry parameter $\epsilon$ are spinors of positive chirality.

If the spacetime is not flat, $\epsilon$ must be a covariantly constant spinor. We are interested in the case when the spacetime is $\mathbb{C} \times \mathbb{M}_{6} \times \mathbb{C}$, where $\mathbb{M}_{6}$ is a Calabi-Yau threefold. In this case, covariantly constant spinors are either zero-forms or top forms on $\mathbb{M}_{6}$ since they are the only forms that are invariant under the holonomy group $\mathrm{SU}(3)$. Hence, using the holomorphic volume form $\Omega_{3}$ of $\mathbb{M}_{6}$, we can write

$$
\epsilon=\epsilon_{-} \mathrm{d} z^{1}+\epsilon_{+} \mathrm{d} z^{5}-\bar{\epsilon}_{+} \Omega_{3}+\bar{\epsilon}_{-} \mathrm{d} z^{1} \wedge \Omega_{3} \wedge \mathrm{d} z^{5} .
$$

We also write $\Psi$ as

$$
\begin{aligned}
\Psi= & -\lambda_{-} \mathrm{d} z^{1}-\lambda_{+} \mathrm{d} z^{5}+\bar{\lambda}_{+} \Omega_{3}-\bar{\lambda}_{-} \mathrm{d} z^{1} \wedge \Omega_{3} \wedge \mathrm{d} z^{5} \\
& +\sum_{k=2}^{4}\left(-\mathrm{i} \bar{\psi}_{+}^{k} \mathrm{~d} z^{k}+\mathrm{i} \bar{\psi}_{-}^{k} \mathrm{~d} z^{1} \wedge \mathrm{d} z^{k} \wedge \mathrm{d} z^{5}+\mathrm{i} \psi_{+}^{k} \iota_{\partial_{k}} \Omega_{3} \wedge \mathrm{d} z^{5}+\mathrm{i} \psi_{-}^{k} \mathrm{~d} z^{1} \wedge \iota_{\partial_{k}} \Omega_{3}\right),
\end{aligned}
$$

where we have chosen complex coordinates $\left(z^{2}, z^{3}, z^{4}\right)$ on $\mathbb{M}_{6}$ such that

$$
\Omega_{3}=\mathrm{d} z^{2} \wedge \mathrm{d} z^{3} \wedge \mathrm{d} z^{4} .
$$

Plugging the above expressions for $\epsilon$ and $\Psi$ into the supersymmetry variation (A.10) of $A$, we get

$$
\begin{aligned}
& \delta A_{1}=-\mathrm{i} \epsilon_{-} \bar{\lambda}_{-}-\mathrm{i} \bar{\epsilon}_{-} \lambda_{-}, \\
& \delta A_{\overline{1}}=\mathrm{i} \epsilon_{+} \bar{\lambda}_{+}+\mathrm{i} \bar{\epsilon}_{+} \lambda_{+}, \\
& \delta A_{5}=-\mathrm{i} \epsilon_{+} \bar{\lambda}_{-}-\mathrm{i} \bar{\epsilon}_{-} \lambda_{+}, \\
& \delta A_{\overline{5}}=-\mathrm{i} \bar{\epsilon}_{+} \lambda_{-}-\mathrm{i} \epsilon_{-} \bar{\lambda}_{+}, \\
& \delta A_{k}=-\bar{\epsilon}_{+} \bar{\psi}_{-}^{k}+\bar{\epsilon}_{-} \bar{\psi}_{+}^{k}, \\
& \delta A_{\bar{k}}=\epsilon_{+} \psi_{-}^{k}-\epsilon_{-} \psi_{+}^{k} .
\end{aligned}
$$


From the supersymmetry variation (A.10) of $\Psi$, we find

$$
\begin{aligned}
& \delta \lambda_{+}=\epsilon_{+}\left(2 F_{1 \overline{1}}-2 F_{5 \overline{5}}-F_{k}^{k}\right)+4 \epsilon_{-} F_{\overline{1} 5}, \\
& \delta \lambda_{-}=-\epsilon_{-}\left(2 F_{1 \overline{1}}-2 F_{5 \overline{5}}+F_{k}^{k}\right)-4 \epsilon_{+} F_{1 \overline{5}}, \\
& \delta \bar{\lambda}_{+}=\bar{\epsilon}_{+}\left(2 F_{1 \overline{1}}+2 F_{5 \overline{5}}+F_{k}^{k}\right)+4 \bar{\epsilon}_{-} F_{\overline{1} \overline{5}}, \\
& \delta \bar{\lambda}_{-}=-\bar{\epsilon}_{-}\left(2 F_{1 \overline{1}}+2 F_{5 \overline{5}}-F_{k}^{k}\right)-4 \bar{\epsilon}_{+} F_{15}, \\
& \delta \psi_{+}^{k}=4 \mathrm{i} \bar{\epsilon}_{-} F_{\overline{1} \bar{k}}+4 \mathrm{i} \bar{\epsilon}_{+} F_{5 \bar{k}}-2 \mathrm{i} \epsilon_{+} \varepsilon^{k l m} F_{l m}, \\
& \delta \psi_{-}^{k}=4 \mathrm{i} \bar{\epsilon}_{+} F_{1 \bar{k}}-4 \mathrm{i} \bar{\epsilon}_{-} F_{\overline{5} \bar{k}}-2 \mathrm{i} \epsilon_{-} \varepsilon^{k l m} F_{l m}, \\
& \delta \bar{\psi}_{+}^{k}=-4 \mathrm{i} \epsilon_{-} F_{\overline{1} k}-4 \mathrm{i} \epsilon_{+} F_{\overline{5} k}+2 \mathrm{i} \bar{\epsilon}_{+} \varepsilon^{k l m} F_{\bar{l} \bar{m}}, \\
& \delta \bar{\psi}_{-}^{k}=-4 \mathrm{i} \epsilon_{+} F_{1 k}+4 \mathrm{i} \epsilon_{-} F_{5 k}+2 \mathrm{i} \bar{\epsilon}_{-} \varepsilon^{k l m} F_{\bar{l} \bar{m}} .
\end{aligned}
$$

Here $\varepsilon^{k l m}, k, l, m=2,3,4$, are the components of a completely antisymmetric tensor on $\mathbb{M}_{6}$, with $\varepsilon^{234}=1$.

We compare these equations with $\mathcal{N}=(2,2)$ supersymmetry transformation laws. ${ }^{8}$ An $\mathcal{N}=(2,2)$ vector multiplet transforms under supersymmetry as

$$
\begin{aligned}
& \delta A_{ \pm}= 2 \mathrm{i} \epsilon_{ \pm} \bar{\lambda}_{ \pm}+2 \mathrm{i} \bar{\epsilon}_{ \pm} \lambda_{ \pm}, \\
& \delta \sigma=-\mathrm{i} \bar{\epsilon}_{+} \lambda_{-}-\mathrm{i} \epsilon_{-} \bar{\lambda}_{+}, \\
& \delta \bar{\sigma}=-\mathrm{i} \epsilon_{+} \bar{\lambda}_{-}-\mathrm{i} \bar{\epsilon}_{-} \lambda_{+}, \\
& \delta \lambda_{+}=-\epsilon_{+}\left(F_{01}-2[\sigma, \bar{\sigma}]-\mathrm{iD}\right)+2 \epsilon_{-} D_{+} \bar{\sigma} \\
& \delta \lambda_{-}= \epsilon_{-}\left(F_{01}-2[\sigma, \bar{\sigma}]+\mathrm{iD}\right)+2 \epsilon_{+} D_{-} \sigma \\
& \delta \bar{\lambda}_{+}=-\bar{\epsilon}_{+}\left(F_{01}+2[\sigma, \bar{\sigma}]+\mathrm{iD}\right)+2 \bar{\epsilon}_{-} D_{+} \sigma \\
& \delta \bar{\lambda}_{-}=\bar{\epsilon}_{-}\left(F_{01}+2[\sigma, \bar{\sigma}]-\mathrm{iD}\right)+2 \bar{\epsilon}_{+} D_{-} \bar{\sigma} \\
& \delta \mathrm{D}=\epsilon_{+}\left(D_{-} \bar{\lambda}_{+}+2\left[\sigma, \bar{\lambda}_{-}\right]\right)+\epsilon_{-}\left(D_{+} \bar{\lambda}_{-}+2\left[\bar{\sigma}, \bar{\lambda}_{+}\right]\right) \\
& \quad \quad-\bar{\epsilon}_{+}\left(D_{-} \lambda_{+}+2\left[\bar{\sigma}, \lambda_{-}\right]\right)-\bar{\epsilon}_{-}\left(D_{+} \lambda_{-}+2\left[\sigma, \lambda_{+}\right]\right) .
\end{aligned}
$$

An $\mathcal{N}=(2,2)$ chiral multiplet transforms as

$$
\begin{aligned}
\delta \varphi & =\epsilon_{+} \psi_{-}-\epsilon_{-} \psi_{+} \cdot \\
\delta \psi_{+} & =2 \mathrm{i} \bar{\epsilon}_{-} D_{+} \varphi+4 \mathrm{i} \bar{\epsilon}_{+} \bar{\sigma} \varphi+2 \epsilon_{+} \mathrm{F}, \\
\delta \psi_{-} & =-2 \mathrm{i} \bar{\epsilon}_{+} D_{-} \varphi-4 \mathrm{i} \bar{\epsilon}_{-} \sigma \varphi+2 \epsilon_{-} \mathrm{F}, \\
\delta \mathrm{F} & =-\mathrm{i} \bar{\epsilon}_{+}\left(D_{-} \psi_{+}+2 \bar{\sigma} \psi_{-}-2 \mathrm{i} \bar{\lambda}_{-} \varphi\right)-\mathrm{i} \bar{\epsilon}_{-}\left(D_{+} \psi_{-}+2 \sigma \psi_{+}+2 \mathrm{i} \bar{\lambda}_{+} \varphi\right) . \\
\delta \bar{\varphi} & =-\bar{\epsilon}_{+} \bar{\psi}_{-}+\bar{\epsilon}_{-} \bar{\psi}_{+} \cdot \\
\delta \bar{\psi}_{+} & =-2 \mathrm{i} \epsilon_{-} D_{+} \bar{\varphi}-4 \mathrm{i} \epsilon_{+} \sigma \bar{\varphi}+2 \bar{\epsilon}_{+} \overline{\mathrm{F}} \\
\delta \bar{\psi}_{-} & =2 \mathrm{i} \epsilon_{+} D_{-} \bar{\varphi}+4 \mathrm{i} \epsilon_{-} \bar{\sigma} \bar{\varphi}+2 \bar{\epsilon}_{-} \overline{\mathrm{F}} \\
\delta \overline{\mathrm{F}} & =-\mathrm{i} \epsilon_{+}\left(D_{-} \bar{\psi}_{+}+2 \sigma \bar{\psi}_{-}+2 \mathrm{i} \lambda_{-} \bar{\varphi}\right)-\mathrm{i} \epsilon_{-}\left(D_{+} \bar{\psi}_{-}+2 \bar{\sigma} \bar{\psi}_{+}-2 \mathrm{i} \lambda_{+} \bar{\varphi}\right) .
\end{aligned}
$$

${ }^{8}$ The following formulas are obtained from $\mathcal{N}=1$ supersymmetry transformations in $3+1$ dimensions by dimensional reduction in the $x^{1}$ - and $x^{2}$-directions. Compared to the formulas in [55], we have made the rescaling $\sigma \rightarrow \sqrt{2} \sigma, \phi \rightarrow \sqrt{2} \phi$ and $\mathrm{F} \rightarrow \sqrt{2} \mathrm{~F}$. 
These are the supersymmetry transformations in Minkowski spacetime $\mathbb{R}^{1,1}$ with coordinates $\left(x^{0}, x^{1}\right)$; we have defined $A_{ \pm}=A_{0} \pm A_{1}, \partial_{ \pm}=\partial_{0} \pm \partial_{1}$ and $D_{ \pm}=\partial_{ \pm}+\left[A_{ \pm},\right]$.

Taking Wick rotation $x^{0} \mapsto-\mathrm{i} x^{2}$ into account, we see that the supersymmetry variations (A.15)-(A.28) are precisely of the form of $\mathcal{N}=(2,2)$ supersymmetry transformations if we identify

$$
\begin{aligned}
\sigma & =A_{\overline{5}}, \\
\mathrm{D} & =\mathrm{i} F_{k}^{k}, \\
\varphi^{k} & =A_{\bar{k}}, \\
\mathrm{~F}^{k} & =-\mathrm{i} \varepsilon^{k l m} F_{l m} .
\end{aligned}
$$

Therefore, the scalars $\varphi^{k}$ of three chiral multiplets, labeled by $k=2,3,4$, are the components of the one-form

$$
\mathcal{A}=A_{\bar{k}} \mathrm{~d} \bar{z}^{\bar{k}}
$$

Let us reduce the theory to eight-dimensional super Yang-Mills theory on $\mathbb{C} \times \mathbb{M}_{6}$. This turns the components $A_{5}, A_{\overline{5}}$ of the gauge field to scalars $\bar{\sigma}, \sigma$. As an $\mathcal{N}=(2,2)$ supersymmetric gauge theory, the target space of the theory is the space of $\mathfrak{h}_{\mathbb{C}}$-valued $(0,1)$-forms on $\mathbb{M}_{6}$, where $\mathfrak{h}$ is the Lie algebra of the gauge group. We need to determine the superpotential.

To conform to the notations used in the main body of the paper, we should relate the $\mathcal{N}=(2,2)$ supersymmetry transformations just described to the supersymmetry transformations (4.9)-(4.21) for the $\Omega$-deformed gauged B-model. The $\Omega$-deformed B-twisted supersymmetry is realized by the following supersymmetry parameters:

$$
\begin{aligned}
& \bar{\epsilon}_{ \pm}= \pm \frac{\mathrm{i}}{2}, \\
& \epsilon_{ \pm}=-\frac{1}{2}\left(V^{1} \pm \mathrm{i} V^{2}\right) .
\end{aligned}
$$

It is easy to identify various fields across the two notations; in particular, we have the identification

$$
\mathrm{F}=-\mathrm{i} \mathrm{F}_{12}
$$

The right-hand side is the component of the two-form auxiliary field $\mathrm{F}$ in the $\Omega$-deformed B-model.

If we normalize the kinetic term of super Yang-Mills theory as

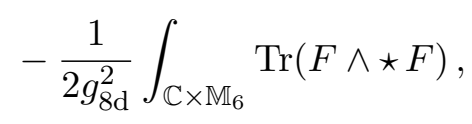

then the part of the B-model action that contains the auxiliary fields $F, \bar{F}$ is

$$
\int_{\mathbb{C} \times \mathbb{M}_{6}}\left(-\frac{2}{g_{8 \mathrm{~d}}^{2}} \operatorname{Tr}(\mathrm{F} \wedge \star \mathrm{F})+\mathrm{F}^{k} \frac{\delta W}{\delta A_{\bar{k}}}-\overline{\mathrm{F}}^{\bar{k}} \frac{\delta \bar{W}}{\delta A_{k}}\right) .
$$

Choosing a basis $\left\{T_{a}\right\}$ of $\mathfrak{h}$ such that $\operatorname{Tr}\left(T_{a} T_{b}\right)=-\delta_{a b}$, we can write the equation of motion for $F$ as

$$
\frac{\delta \bar{W}}{\delta A_{k}^{a}}=\frac{2}{g_{8 d}^{2}} \mathrm{~F}_{12}^{k a}=\frac{2}{g_{8 d}^{2}} \varepsilon^{k l m} F_{l m}^{a} .
$$


Since

$$
\begin{aligned}
\delta \int_{\mathbb{M}_{6}} \bar{\Omega}_{3} \wedge \operatorname{CS}(\overline{\mathcal{A}}) & =2 \int_{\mathbb{M}_{6}} \bar{\Omega}_{3} \wedge \Omega_{3} \operatorname{Tr}\left(\varepsilon^{k l m} \delta A_{k} F_{l m}\right) \\
& =-16 \mathrm{i} \int_{\mathbb{M}_{6}} \star \operatorname{Tr}\left(\varepsilon^{k l m} \delta A_{k} F_{l m}\right),
\end{aligned}
$$

we deduce

$$
W=\frac{\mathrm{i}}{8 g_{8 \mathrm{~d}}^{2}} \int_{\mathbb{M}_{6}} \Omega_{3} \wedge \mathrm{CS}(\mathcal{A}) .
$$

In section 5.4, we make a different choice for the chiral multiplet scalars. There, the real coordinates on $\mathbb{M}_{6}$ are $\left(x^{3}, x^{4}, x^{5}, x^{8}, x^{9}, x^{10}\right)$, and we treat $A_{\overline{3}}, A_{\overline{4}}, A_{\overline{4}}$ in the complex coordinates $z^{3}=\left(x^{3}-\mathrm{i} x^{8}\right) / 2, z^{4}=\left(x^{4}-\mathrm{i} x^{9}\right) / 2$ and $z^{5}=\left(x^{5}-\mathrm{i} x^{10}\right) / 2$ as chiral multiplet scalars. This means that the volume form of $\mathbb{M}_{6}$ is $\left(-\mathrm{d} x^{3} \wedge \mathrm{d} x^{8}\right) \wedge\left(-\mathrm{d} x^{4} \wedge \mathrm{d} x^{9}\right) \wedge\left(-\mathrm{d} x^{5} \wedge\right.$ $\left.\mathrm{d} x^{10}\right)$, and the holomorphic volume form $\Omega_{3}=\mathrm{d} z^{2} \wedge \mathrm{d} z^{3} \wedge \mathrm{d} z^{4}$ is normalized in such a way that $\Omega_{3} \wedge \bar{\Omega}_{3}$ is $-\mathrm{i} / 8$ of the volume form. The equation for motion for the auxiliary field is modified to $\mathrm{F}^{k}=-2 \mathrm{i} \varepsilon^{k l m} F_{l m}$, but in the action there is an extra factor of $1 / 4$ multiplying the quadratic term in the auxiliary fields, so we obtain the superpotential (5.42).

Open Access. This article is distributed under the terms of the Creative Commons Attribution License (CC-BY 4.0), which permits any use, distribution and reproduction in any medium, provided the original author(s) and source are credited.

\section{References}

[1] S. Gukov and E. Witten, Branes and quantization, Adv. Theor. Math. Phys. 13 (2009) 1445 [arXiv:0809.0305] [INSPIRE].

[2] J. Yagi, $\Omega$-deformation and quantization, JHEP 08 (2014) 112 [arXiv:1405.6714] [INSPIRE].

[3] D. Gaiotto and M. Rapčák, Vertex algebras at the corner, JHEP 01 (2019) 160 [arXiv: 1703.00982] [INSPIRE].

[4] K. Costello and D. Gaiotto, Vertex operator algebras and $3 d \mathcal{N}=4$ gauge theories, JHEP 05 (2019) 018 [arXiv: 1804.06460] [INSPIRE].

[5] C. Beem, M. Lemos, P. Liendo, W. Peelaers, L. Rastelli and B.C. van Rees, Infinite chiral symmetry in four dimensions, Commun. Math. Phys. 336 (2015) 1359 [arXiv:1312.5344] [INSPIRE].

[6] J. Oh and J. Yagi, Chiral algebras from $\Omega$-deformation, JHEP 08 (2019) 143 [arXiv: 1903.11123] [INSPIRE].

[7] S. Jeong, SCFT/VOA correspondence via $\Omega$-deformation, JHEP 10 (2019) 171 [arXiv: 1904.00927] [INSPIRE].

[8] E. Witten, Analytic continuation of Chern-Simons theory, AMS/IP Stud. Adv. Math. 50 (2011) 347 [arXiv: 1001.2933] [inSPIRE].

[9] E. Witten, A new look at the path integral of quantum mechanics, in Surveys in differential geometry. Volume XV. Perspectives in mathematics and physics, vol. 15 of Surv. Differ. Geom., p. 345. Int. Press, Somerville, MA, U.S.A. (2011), [arXiv:1009.6032] [INSPIRE]. 
[10] E. Witten, Fivebranes and knots, Quantum Topol. 3 (2012) 1 arXiv:1101.3216 [INSPIRE].

[11] T. Dimofte, S. Gukov and L. Hollands, Vortex counting and Lagrangian 3-manifolds, Lett. Math. Phys. 98 (2011) 225 [arXiv:1006.0977] [InSPIRE].

[12] Y. Terashima and M. Yamazaki, $\mathrm{SL}(2, \mathbb{R})$ Chern-Simons, Liouville, and gauge theory on duality walls, JHEP 08 (2011) 135 [arXiv:1103.5748] [INSPIRE].

[13] Y. Terashima and M. Yamazaki, Semiclassical analysis of the 3d/3d relation, Phys. Rev. D 88 (2013) 026011 [arXiv: 1106.3066] [INSPIRE].

[14] S. Cecotti, C. Cordova and C. Vafa, Braids, walls, and mirrors, arXiv:1110.2115 [InSPIRE].

[15] T. Dimofte and S. Gukov, Chern-Simons theory and S-duality, JHEP 05 (2013) 109 [arXiv:1106.4550] [INSPIRE].

[16] T. Dimofte, D. Gaiotto and S. Gukov, Gauge theories labelled by three-manifolds, Commun. Math. Phys. 325 (2014) 367 [arXiv:1108.4389] [INSPIRE].

[17] T. Dimofte, D. Gaiotto and S. Gukov, 3-manifolds and 3d indices, Adv. Theor. Math. Phys. 17 (2013) 975 [arXiv:1112.5179] [inSPIRE].

[18] Y. Luo, M.-C. Tan, J. Yagi and Q. Zhao, $\Omega$-deformation of B-twisted gauge theories and the 3d-3d correspondence, JHEP 02 (2015) 047 [arXiv: 1410.1538] [INSPIRE].

[19] N.A. Nekrasov and S.L. Shatashvili, Supersymmetric vacua and Bethe ansatz, Nucl. Phys. B Proc. Suppl. 192/193 (2009) 91 [arXiv:0901.4744] [INSPIRE].

[20] N.A. Nekrasov and S.L. Shatashvili, Quantum integrability and supersymmetric vacua, Prog. Theor. Phys. Suppl. 177 (2009) 105 arXiv:0901.4748 [INSPIRE].

[21] H.-Y. Chen, N. Dorey, T.J. Hollowood and S. Lee, A new 2d/4d duality via integrability, JHEP 09 (2011) 040 [arXiv: 1104.3021] [INSPIRE].

[22] N. Nekrasov and V. Pestun, Seiberg-Witten geometry of four dimensional $N=2$ quiver gauge theories, arXiv:1211.2240 [INSPIRE].

[23] N. Nekrasov, V. Pestun and S. Shatashvili, Quantum geometry and quiver gauge theories, Commun. Math. Phys. 357 (2018) 519 [arXiv: 1312.6689] [INSPIRE].

[24] M. Yamazaki, Quivers, YBE and 3-manifolds, JHEP 05 (2012) 147 [arXiv:1203.5784] [INSPIRE].

[25] J. Yagi, Quiver gauge theories and integrable lattice models, JHEP 10 (2015) 065 [arXiv: 1504.04055] [INSPIRE].

[26] K. Costello, Supersymmetric gauge theory and the Yangian, arXiv:1303.2632 [INSPIRE].

[27] K. Costello, Integrable lattice models from four-dimensional field theories, Proc. Symp. Pure Math. 88 (2014) 3 [arXiv: 1308.0370] [INSPIRE].

[28] K. Costello, E. Witten and M. Yamazaki, Gauge theory and integrability, I, ICCM Not. 6 (2018) 46 [arXiv: 1709.09993] [INSPIRE].

[29] K. Costello, E. Witten and M. Yamazaki, Gauge theory and integrability, II, ICCM Not. 6 (2018) 120 [arXiv: 1802.01579] [INSPIRE].

[30] K. Costello and J. Yagi, Unification of integrability in supersymmetric gauge theories, arXiv: 1810.01970 [INSPIRE].

[31] E. Witten, Topological sigma models, Commun. Math. Phys. 118 (1988) 411 [INSPIRE]. 
[32] A. Kapustin and D. Orlov, Remarks on A-branes, mirror symmetry, and the Fukaya category, J. Geom. Phys. 48 (2003) 84 [hep-th/0109098] [inSPIRE].

[33] M. Blau and G. Thompson, Aspects of $N_{T} \geq 2$ topological gauge theories and D-branes, Nucl. Phys. B 492 (1997) 545 [hep-th/9612143] [INSPIRE].

[34] J.P. Yamron, Topological actions from twisted supersymmetric theories, Phys. Lett. B 213 (1988) 325 [INSPIRE].

[35] N. Marcus, The other topological twisting of $N=4$ Yang-Mills, Nucl. Phys. B 452 (1995) 331 [hep-th/9506002] [INSPIRE].

[36] A. Kapustin and E. Witten, Electric-magnetic duality and the geometric Langlands program, Commun. Num. Theor. Phys. 1 (2007) 1.

[37] N.A. Nekrasov, Seiberg-Witten prepotential from instanton counting, Adv. Theor. Math. Phys. 7 (2003) 831 [hep-th/0206161] [INSPIRE].

[38] N. Nekrasov and A. Okounkov, Seiberg-Witten theory and random partitions, in The unity of mathematics, vol. 244, pp. 525-596, (2006), hep-th/0306238 [DOI] [INSPIRE].

[39] L. Rozansky and E. Witten, Hyper-Kähler geometry and invariants of three-manifolds, Selecta Math. 3 (1997) 401 [hep-th/9612216] [INSPIRE].

[40] A. Kapustin, Holomorphic reduction of $\mathcal{N}=2$ gauge theories, Wilson-'t Hooft operators, and S-duality, hep-th/0612119 [INSPIRE].

[41] N. Nekrasov and E. Witten, The Omega deformation, branes, integrability and Liouville theory, JHEP 09 (2010) 092 [arXiv: 1002. 0888] [INSPIRE].

[42] J.M. Baptista, Twisting gauged non-linear sigma-models, JHEP 02 (2008) 096 [arXiv: 0707.2786] [INSPIRE].

[43] C. Vafa, Topological Landau-Ginzburg models, Mod. Phys. Lett. A 6 (1991) 337 [INSPIRE].

[44] E. Witten, Mirror manifolds and topological field theory, in Essays on mirror manifolds, Int. Press, Hong Kong, (1992), pp. 120-158, hep-th/9112056 [INSPIRE].

[45] M. Ashwinkumar, M.-C. Tan and Q. Zhao, Branes and categorifying integrable lattice models, Adv. Theor. Math. Phys. 24 (2020) 1 [arXiv: 1806. 02821] [INSPIRE].

[46] M. Ashwinkumar and M.-C. Tan, Unifying lattice models, links and quantum geometric Langlands via branes in string theory, arXiv:1910.01134 [INSPIRE].

[47] E. Witten, Chern-Simons gauge theory as a string theory, Prog. Math. 133 (1995) 637 [hep-th/9207094] [INSPIRE].

[48] K. Costello, $M$-theory in the $\Omega$-background and 5-dimensional non-commutative gauge theory, arXiv: 1610.04144 [INSPIRE].

[49] E. Witten, Supersymmetry and Morse theory, J. Diff. Geom. 17 (1982) 661 [InSPIRE].

[50] N. Nekrasov, Tying up instantons with anti-instantons, arXiv:1802.04202 [INSPIRE].

[51] N. Hitchin, Monopoles, minimal surfaces and algebraic curves, vol. 105 of Séminaire de Mathématiques Supérieures [Seminar on Higher Mathematics], Presses de l'Université de Montréal, Montreal, QC, (1987).

[52] G. Thompson, On the generalized Casson invariant, Adv. Theor. Math. Phys. 3 (1999) 249 [hep-th/9811199] [INSPIRE]. 
[53] A. Kapustin, K. Setter and K. Vyas, Surface operators in four-dimensional topological gauge theory and Langlands duality, arXiv:1002.0385 [INSPIRE].

[54] D. Gaiotto, Twisted compactifications of $3 d \mathcal{N}=4$ theories and conformal blocks, JHEP 02 (2019) 061 [arXiv: 1611.01528] [INSPIRE].

[55] E. Witten, Phases of $N=2$ theories in two dimensions, Nucl. Phys. B 403 (1993) 159 [hep-th/9301042] [INSPIRE].

[56] D. Gaiotto, G.W. Moore and A. Neitzke, Wall-crossing, Hitchin systems, and the WKB approximation, Adv. Math. 234 (2013) 239 [arXiv:0907.3987] [INSPIRE].

[57] C. Elliott, P. Safronov and B.R. Williams, A taxonomy of twists of supersymmetric Yang-Mills theory, arXiv:2002.10517 [INSPIRE]. 